San Jose State University

SJSU ScholarWorks

Master's Theses

Master's Theses and Graduate Research

1996

\title{
Calculations and measurements of neutron arrival time for neutron detectors at SLAC
}

Kaur Rupinder

San Jose State University

Follow this and additional works at: https://scholarworks.sjsu.edu/etd_theses

\section{Recommended Citation}

Rupinder, Kaur, "Calculations and measurements of neutron arrival time for neutron detectors at SLAC" (1996). Master's Theses. 1313.

DOI: https://doi.org/10.31979/etd.6efr-ran8

https://scholarworks.sjsu.edu/etd_theses/1313

This Thesis is brought to you for free and open access by the Master's Theses and Graduate Research at SJSU ScholarWorks. It has been accepted for inclusion in Master's Theses by an authorized administrator of SJSU ScholarWorks. For more information, please contact scholarworks@sjsu.edu. 


\section{INFORMATION TO USERS}

This manuscript has been reproduced from the microfilm master. UMI films the text directly from the original or copy submitted. Thus, some thesis and dissertation copies are in typewriter face, while others may be from any type of computer printer.

The quality of this reproduction is dependent upon the quality of the copy submitted. Broken or indistinct print, colored or poor quality illustrations and photographs, print bleedthrough, substandard margins, and improper alignment can adversely affect reproduction.

In the unlikely event that the author did not send UMI a complete manuscript and there are missing pages, these will be noted. Also, if unauthorized copyright material had to be removed, a note will indicate the deletion.

Oversize materials (e.g., maps, drawings, charts) are reproduced by sectioning the original, beginning at the upper left-hand comer and continuing from left to right in equal sections with small overlaps. Each original is also photographed in one exposure and is included in reduced form at the back of the book.

Photographs included in the original manuscript have been reproduced xerographically in this copy. Higher quality 6" $\times$ 9" black and white photographic prints are available for any photographs or illustrations appearing in this copy for an additional charge. Contact UMI directly to order.

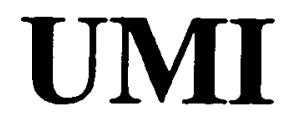

A Bell \& Howell Information Company 



\title{
CALCULATIONS AND MEASUREMENTS OF NEUTRON ARRIVAL TIME FOR NEUTRON DETECTORS AT SLAC
}

\author{
A Thesis \\ Presented to \\ The Faculty of the Nuclear Science Program \\ San Jose State University
}

\author{
In Partial Fulfillment \\ of the Requirements for the Degree \\ Master of Science
}

by

Rupinder Kaur

August 1996 
UMI Number: 1381424

\section{Copyright 1996 by \\ Raur, Rupinder}

All rights reserved.

UMI Microform 1381424

Copyright 1996, by UMI Company. All rights reserved.

This microform edition is protected against unauthorized copying under Title 17, United States Code.

\section{UMI \\ 300 North Zeeb Road \\ Ann Arbor, MI 48103}


Rupinder Kaur

ALL RIGHTS RESERVED 
APPROVED FOR THE DEPARTMENT OF NUCLEAR SCIENCE 4 is Tach

Dr. Allen B. Tucker

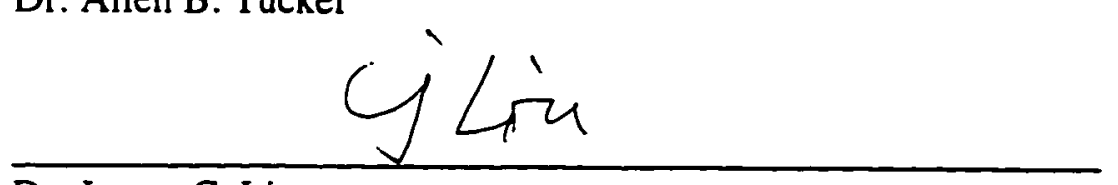

Dr. James C. Diu

S.H. Rotor i

Dr. Sayed H Rokni

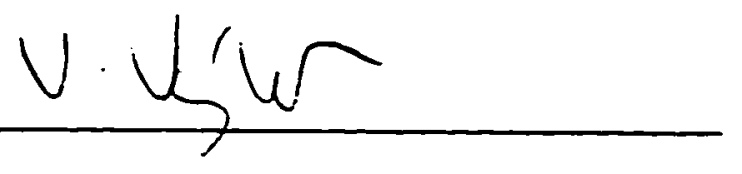

Dr. Vashek Vylet

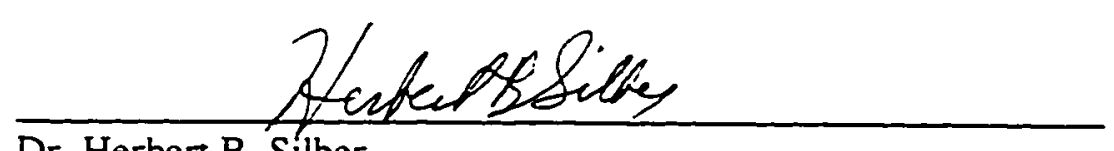

Dr. Herbert B. Silver

APPROVED FOR THE UNIVERSITY

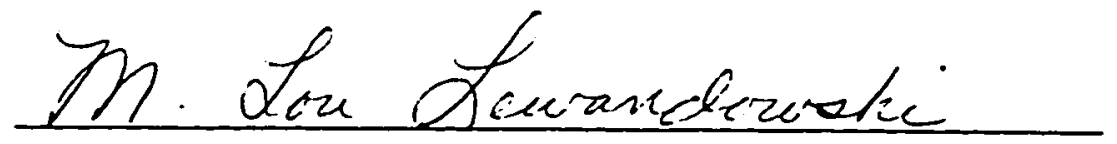




\section{ABSTRACT \\ CALCULATIONS AND MEASUREMENTS OF NEUTRON ARRIVAL TIME FOR NEUTRON DETECTORS AT SLAC. \\ by Rupinder Kaur}

The distributions of neutron arrival time (the time between the neutron being generated in a target and the time it is detected by the sensor) are important for the dead time corrections of the neutron detectors in pulsed radiation field. Calculations using MCNP and measurements of the neutron arrival time distributions were made for the moderated $\mathrm{BF}_{3}$ detector, Anderson-Braun remmeter, and Bonner Sphere LiI detectors. Neutron sources defined in these calculations include monoenergetic neutrons from a few $\mathrm{eV}$ to $20 \mathrm{MeV}$, giant resonance neutrons, and the measured 'equilibrium' neutron spectrum of Final Focus Test Beam (FFTB) at SLAC. The results show an exponential behavior of the neutron arrival time distributions. Comparisons between calculations and measurements show close agreement for $10^{\prime \prime}$ Bonner Sphere detector and a maximum difference of about $20 \%$ for moderated $\mathrm{BF}_{3}$ detector and $\mathrm{AB}$ remmeter. The cause of the discrepancy for 2" Bonner Sphere detector is also explained. 


\section{ACKNOWLEDGMENTS}

I would like to thank Dr. J. C. Liu of SLAC for giving me the opportunity to work on this project. I greatly appreciate his supervision, guidance and deep involvement in this work. I am thankful to Dr. Allen B. Tucker and Dr. Herbert B. Silber of SJSU for

reviewing and approving my work. Thanks are also due to all thesis committee members for their helpful reviews.

Special thanks go to Dr. Sayed H Rokni of SLAC for his help in measurement portion and to Dr. Vashek Vylet of SLAC for the valuable discussions on many aspects throughout this work. I would also like to thank Mr. Ron Seefred of SLAC for his help in the electronics set up.

I express my indebtedness to my parents for their support and efforts in all the accomplishments of my life. I am extremely grateful to my husband Sukhdev Singh for his never-ending encouragement, understanding, support, love and care. This thesis is dedicated to my family. 


\section{TABLE OF CONTENTS}

Chapter

1. Introduction

1.1 Introduction

1.2 Dead Time Correction

1.3 Neutron Production at SLAC

1.4 Objective

1.5 Summary

2. Monte Carlo Calculations 11

2.1 Introduction 11

2.2 Geometry of detectors 13

2.2.1 Moderated $\mathrm{BF}_{3}$ detector 13

2.2.2 Anderson-Braun remmeter 16

2.2.3 Bonner Sphere detectors 19

2.3 MCNP input file $\quad 22$

2.4 MCNP output file $\quad 24$

2.5 Neutron sources 25

$\begin{array}{ll}\text { 3. } & \text { Measurements } \\ \end{array}$

3.1 Introduction 29

3.2 Electronics 31

4. Results and Discussions 39

4.1 Calculations 39

4.1.1 Moderated $\mathrm{BF}_{3}$ detector results 39

4.1.2 Anderson-Braun remmeter results 44

4.1.3 Bonner Sphere results 48

4.2 Measurements and comparisons $\quad 59$

5. Summary and Conclusions 65

5.1 Summary 65

5.2 Conclusions 66

5.3 Further studies 68

$\begin{array}{ll}\text { References } & 69\end{array}$ 


\section{APPENDICES}

Al $\mathrm{MiCl}$ P input file of the moderated $\mathrm{BF}_{3}$ delector for monoenergetic neutrons $(20 \mathrm{MeV})$.

A2 MCNP input file of the moderated $\mathrm{BF}_{3}$ detector for GRNs. 71

A3 MCNP input file of the moderated $\mathrm{BF}_{3}$ detector for FFTB measured 72 neutron spectrum.

Bl MCNP input file of the Anderson-Braun remmeter for monoenergetic $\quad 73$ neutrons (1 MeV).

B2 MCNP input file of the Anderson-Braun remmeter for GRNs. 74

B3 MCNP input file of the Anderson-Braun remmeter for FFTB measured 75

$\mathrm{Cl}$ MCNP input file of the 2" Bonner Sphere for monoenergetic 76 neutrons ( $1 \mathrm{MeV})$.

C2 MCNP input file of the 8" Bonner Sphere for GRNs. 77

C3 MCNP input file of the 12" Bonner Sphere for FFTB measured 78 neutron spectrum.

D A portion of MCNP output file of the moderated $\mathrm{BF}_{3}$ detector for $\quad 79$ $1 \mathrm{MeV}$ neutrons.

E MCNP-calculated response of the moderated $\mathrm{BF}_{3}$ detector and Anderson-Braun remmeter for various monoenergetic neutrons, GRN and FFTB measured neutron spectrum. 


\section{LIST OF TABLES}

Tabie

page

1 MCNP calculated response of Bonner Sphere detectors of 2" and 3" diameters for various monoenergetic neutrons, GRNs, and FFTB measured neutron spectrum.

2 MCNP calculated response of Bonner Sphere detectors of 5 " and 8" diameters for various monoenergetic neutrons, GRNs, FFTB measured neutron spectrum.

3 MCNP calculated response of Bonner Sphere detectors of 10 " and 12" diameters for various monoenergetic neutrons, GRNs, FFTB measured neutron spectrum.

4 Mean neutron arrival time for FFTB spectrum 


\section{LIST OF FIGURES}

Figure

Page

2.1 Schematic drawing of the moderated $\mathrm{BF}_{3}$ detector. 14

$\begin{array}{ll}\text { 2.2a Schematic drawing of the Anderson-Braun remmeter. } & 17\end{array}$

2.2b The Anderson-Braun remmeter and its components. 18

2.3a Schematic drawing of the Bonner Sphere detector probe. 20

2.3b Schematic drawing of the Bonner Sphere detector assembly. 21

2.4 MCNP calculated GRN spectrum using parameter ' $a$ ' $=1 \mathrm{MeV}$ in formula $P(E)=C E \exp (-E / a)$. 26

2.5 Measured neutron spectrum for FFTB-'hot spot' location. 27

3.1a Schematic drawing of the locations of the detectors for the 30 measurements outside the shielding of beam dump.

3.1b The photograph showing the location of moderated $\mathrm{BF}_{3}$ detector, 32 reference moderated $\mathrm{BF}_{3}$ detector, $\mathrm{AB}$ remmeter, and the paddle outside the FFTB shielding.

3.1c The photograph showing the location of the Bonner Sphere detector relative to the FFTB concrete shielding and the iron muon shielding.

3.2 Schematic drawing of the electronics setup for the counting measurements made outside the shielding of FFTB beam dump.

3.3a The photograph of the oscilloscope screen displaying the signal from 36 the paddle and the position of the gate.

3.3b The photograph of the oscilloscope screen showing 37 movement of the gate.

4. la-b MCNP calculated neutron arrival time distribution in the moderated $\mathrm{BF}_{3}$ detector (a) for $0.6 \mathrm{eV}-100 \mathrm{eV}$

(b) for $1 \mathrm{keV}-20 \mathrm{MeV}$. 
Figure

4. Ic-d MCNP calculated neutron arrival time distribution in the moderated $\mathrm{BF}_{3}$ detector (c) for $500 \mathrm{keV}-2 \mathrm{MieV}$

(d) for GRN and FFTB measured neutron spectrum.

4.1e MCNP-calculated response of the moderated $\mathrm{BF}_{3}$ detector for the neutrons incident from the side.

4.2a-b MCNP-calculated neutron arrival time distribution in the Anderson-Braun remmeter for monoenergetic neutrons (a) for $10 \mathrm{eV}-2 \mathrm{MeV}$ (b) for $500 \mathrm{keV}-10 \mathrm{MeV}$.

4.2c MCNP-calculated neutron arrival time distribution in the Anderson-Braun remmeter for GRN and FFTB measured neutron spectrum compared with that for $1 \mathrm{MeV}$ neutrons.

4.2d MCNP-calculated response of the Anderson-Braun remmeter.

4.3a-b MCNP-calculated neutron arrival time distribution in the 2" Bonner Sphere detector for monoenergetic neutrons (a) for $0.1 \mathrm{eV}-100 \mathrm{eV}$ (b) for $100 \mathrm{eV}-1 \mathrm{MeV}$.

4.3c MCNP-calculated neutron arrival time distribution in the 2" Bonner Sphere detector for GRN and FFTB measured neutron spectrum compared with that for $1 \mathrm{MeV}$ neutrons.

4.4a-b MCNP-calculated neutron arrival time distribution in the

3" Bonner Sphere detector (a) for monoenergetic neutrons

(b) for GRN and FFTB measured neutron spectrum compared with that for $1 \mathrm{MeV}$ neutrons.

4.5a-b MCNP-calculated neutron arrival time distribution in the $5 \mathrm{n}$. Bonner Sphere detector (a) for monoenergetic neutrons.

(b) for GRN and FFTB measured neutron spectrum compared with that for $1 \mathrm{MeV}$ neutrons.

4.6a-b MCNP-calculated neutron arrival time distribution in the

8 " Bonner Sphere detector (a) for monoenergetic neutrons

(b) for GRN and FFTB measured neutron spectrum. 
Figure $\quad$ Page

4.7a-b MCNP-calculated neutron arrival time distribution in the 54

10" Bonner Sphere detector (a) for monoenergetic neutrons

(b) for GRN and FFTB measured neutron spectrum compared.

4.8a-b MCNP-calculated neutron arrival time distribution in the

12" Bonner Sphere detector (a) for monoenergetic neutrons.

(b) for GRN and FFTB measured neutron spectrum compared

4.9a-b A comparison between the MCNP-calculations and the measurement of fractional cumulative counts versus the neutron arrival time for the FFTB spectrum incident on the moderated $\mathrm{BF}_{3}$ detector (a) a semilog plot (b) a linear plot.

4.10a-b A comparison between the MCNP-calculations and the measurement of fractional cumulative counts versus the neutron arrival time for the FFTB spectrum incident on the Anderson-Braun remmeter (a) a semilog plot (b) a linear plot.

4.1 la-b A comparison between the MCNP-calculations and the measurement of fractional cumulative counts versus the neutron arrival time for the FFTB spectrum incident on the 2" Bonner Sphere detector (a) a semilog plot (b) a linear plot.

4.12a-b A comparison between the MCNP-calculations and the measurement of fractional cumulative counts versus the neutron arrival time for the FFTB spectrum incident on the 10" Bonner Sphere detector (a) a semilog plot (b) a linear plot. 


\section{INTRODUCTION}

\subsection{Introduction}

Neutrons, being neutral particles, can be detected only through nuclear reactions that convert them to energetic charged particles such as protons or alpha particles. Any neutron detector, therefore, would include: a material for this conversion and a charged particle detector. Detection of neutrons imposes a great challenge for various reasons. Typical neutron fields span many decades of energy $(0.1 \mathrm{eV}$ to $1 \mathrm{GeV})$. For the reactions of interest, the neutron cross section depends strongly on neutron energy. For example, the thermal neutron $(0.025 \mathrm{eV})$ cross section for ${ }^{10} \mathrm{~B}(\mathrm{n}, \alpha)^{7} \mathrm{Li}$ is 3840 barns. The cross section value drops rapidly with increasing neutron energy and it is proportional to the reciprocal of neutron velocity $(1 / \mathrm{v})$. At $\mid \mathrm{MeV}$ neutron energy, the cross section for this reaction reduces to a mere 0.2 barns (Knoll 1989). Various detection technologies have been developed in different neutron energy regions.

Slow neutrons (below about $0.5 \mathrm{eV}$ ) are relatively simple to detect by neutron capture in some materials due to their large cross section. Fast neutrons may be detected by slowing them down using a moderator before they reach the thermal neutron sensor. The process in which a fast neutron loses most of its initial kinetic energy (in a series of elastic collisions) to become thermal neutron is called 'moderation.' This can be best achieved by using hydrogenous materials such as polyethylene and paraffin. Hydrogen is a very suitable moderator since it can absorb the largest fraction of neutron energy per elastic

\footnotetext{
In particular. for hydrogen scattering, the average energy loss is one half of the initial neutron energy.
} 
collision. A thermal neutron detector is surrounded by a moderator to form a fast neutron detector. Examples of such moderator type detectors include an Anderson-Braun (AB) remmeter and Bonner Sphere detectors.

For most applications, the detectors are operated in pulse mode ${ }^{*}$. Any detector system operated in pulse mode has some dead time in which there is always some probability that the true event will be lost. The dead time of $\mathrm{BF}_{3}$-based counters is a few $\mu$ s. Therefore, the data analysis must include a dead time correction factor to get an accurate count rate.

In a pulsed radiation field such as that in an accelerator facility like Stanford Linear Accelerator Center (SLAC), the radiation measurements can also be affected by the duty factor of the beam. The more seriously affected instruments include GM counters, proportional counters, bare $\mathrm{BF}_{3}$ counters and scintillation counters. The detectors with moderators are less prone to this effect, because the signal is delayed by the long neutron arrival time (several hundreds of $\mu \mathrm{s}$ ). The arrival time of neutrons in such detectors does affect the dead time correction factor.

\subsection{Dead Time Correction}

To compensate for the loss of counts due to the dead time of the detector, a correction factor must be incorporated in the measured count rate. Two models for the dead time behavior of a counting system have been in use; paralyzable model and non-paralyzable

\footnotetext{
- In pulse mode operation. the measurement instrumentation is designed to record each individual quantum of radiation that interacts in the detector.

- Duty factor is defined as the fraction of time during which an accelerator delivers a useful beam.
} 
model (Knoll 1989). In the paralyzable model, the dead periods are not always of fixed length. The true counting rate $R_{\mathfrak{l}}$ is calculated as:

$$
R_{E}=R e^{-R T_{0}}
$$

where $\mathrm{R}=$ measured counting rate and $T_{d}=$ dead time of the detector.

In the non-paralyzable model, a fixed dead time $T_{d}$ is assumed and the true counting rate $R_{\ell}$ is calculated as:

$$
R_{E}=\frac{R}{I-R I_{\epsilon}}
$$

These models represent an idealized behavior. Either of these may resemble the response of a real counting system. Equations (1) and (2) assume that the detector is being irradiated from a continuos radiation source. If the field is pulsed (i.e., short pulses repeated at a constant frequency), the appropriate correction depends on the relative value of the detector dead time $T_{d}$ and the beam pulse length $T_{p}$. For most accelerator beams $\left(T_{p} \approx 1 \mu s\right)$ and most health physics neutron instruments $\left(T_{d} \approx a\right.$ few $\left.\mu s\right)$ where $T_{p}<T_{d}$, the following equation should be used (Liu 1991):

$$
R_{\varepsilon}=R-P \ln \left(1-\frac{R}{P}\right)
$$


where $\mathbf{P}=$ pulse repetition rate.

If $T_{p}>T_{d}$, then the following equation should be used (Liu 1991):

$$
R_{E}=\frac{R}{1-\frac{R T_{d}}{P T_{p}}\left(1-\frac{1}{2} \frac{T_{d}}{T_{p}}\right)}
$$

where $P T_{p}=$ duty factor.

In situations where moderating type detectors are used for detecting pulsed neutron fields, equation (3) is not valid. Due to moderation and diffusion, short pulses of neutrons (smaller $T_{\mathrm{p}}$ ) are stretched over a longer period of time called the neutron arrival time, which could be a few hundreds of microseconds $\left(T_{p}>>T_{d}\right)$. Therefore, equation (4) should be used. In this study, the arrival time of a neutron has been defined as the time between the neutron incident on the surface of the detector and the neutron detected by the sensor. It is the sum of the moderation time and the diffusion time ${ }^{\top}$. The neutron arrival time is generally dominated by the diffusion time. The signal recorded by the thermal neutron sensor is delayed with respect to the beam pulse. Therefore, effective beam pulse length in equation (4) should not be determined only by the physical $T_{p}$ value. In this case, $T_{p}$ is replaced with $T_{m}$ (mean arrival time) and since $T_{m}>T_{d}$, the

\footnotetext{
- The time for a fast neutron to slow down in the moderator to become a thermal neutron.

- The time for a thermal neutron to diffuse in the moderator and reach the sensor.
} 
parenthetical term in equation (4) is neglected with small error. The equation (4) then becomes equation (5):

$$
R_{e}=\frac{R}{1-\frac{R T_{d}}{P T_{m}}}
$$

It is assumed in equation (5) that the detected neutrons are uniformly distributed in time $T_{m}$. $T_{m}$ is called the mean moderation time by Jenkins (1979). In fact, $T_{m}$ should be referred to the mean arrival time as the delay of the signal is caused by both moderation and diffusion. In this study, $T_{m}$ is called the mean arrival time or the $T$-values.

According to measurements (Ash 1977), the neutron arrival time distribution is close to an exponential distribution. Therefore, the inverse of the slope of the arrival time distribution curve is the mean arrival time $T_{m}$. By incorporating this fact in equation (5) for paralyzable model, the true count rate can be calculated as follows (Liu 1991):

$$
\begin{aligned}
& \qquad R_{\mathrm{t}}=R I \ln \frac{I}{r-1} \\
& \text { where } \quad I=\frac{P T_{m}}{R T_{c}}
\end{aligned}
$$


The parameter $r$ is the ratio of maximum counting rate that can be measured $\left(\mathrm{PT}_{\mathrm{m}} / \mathrm{T}_{\mathrm{d}}\right)$ to the measured counting rate $(\mathrm{R})$.

The spread of the signal due to moderation and diffusion has made the measurements of neutrons less prone to the dead time losses in pulsed fields. In the absence of moderator, the detector would utmost record a single signal pulse per beam pulse. The dramatic influence of the moderator on the dead time correction is illustrated by the following example. For a proton recoil counter, at a pulse repetition rate of $10 \mathrm{~Hz}$, a correction factor is about 1.5 at a measured counting rate of only 2 counts/sec. On the other hand, for the moderated $\mathrm{BF}_{3}$ detector surrounded by $6.3 \mathrm{~cm}$ of paraffin, a correction factor of 1.5 is required for a high count rate of $\approx 500$ counts/sec at $10 \mathrm{~Hz}$ (Patterson 1973).

\subsection{Neutron Production at SLAC}

SLAC has a two-mile-long linear accelerator that accelerates electron and positron beams up to an energy of $50 \mathrm{GeV}$. These beams can be sent to different experimental facilities. One of such facilities is the Final Focus Test Beam (FFTB). The measurements for the present work have been made outside the shielding of the FFTB beam dump.

When a very high energy electron beam interacts with matter, only a small fraction of energy is dissipated through heat or ionization. The large fraction of the energy is spent in the production of high energy photons by bremsstrahlung. The fraction of the kinetic energy of the incident electrons converted to bremsstrahlung is a function of incident 
electron energy $\left(E_{o}\right)$ that increases with the increasing $E_{o}$. The bremsstrahlung photons, in turn, can undergo pair production to produce electron and positron pairs or compton collisions to produce electrons. These new secondary electrons and positrons radiate even more photons and the process goes on until eventually the energies of particles become low. This process is known as electromagnetic (EM) shower.

Neutrons are produced in any material struck by the electron or bremsstrahlung beams of energies above a certain threshold (for example, 4 to $6 \mathrm{MeV}$ for heavy nuclei). It is the photons that release the neutrons by photonuclear reactions rather than the direct interaction of electrons. The mechanisms by which neutrons are produced are as follows (IAEA 1979):

\section{Giant resonance}

For photon energy above a certain threshold and below $30 \mathrm{MeV}$, neutron production results primarily from a process known as giant resonance. The electric field of a photon transfers its energy to nucleus by inducing an oscillation in which protons as a group moves opposite to the neutrons as a group. Neutrons emitted in the decay of this nuclear excitation are called Giant Resonance Neutrons (GRN). The average energy of GRN is in the order of a few MeVs.

\section{Pseudo-deuteron}

At photon energies above $30 \mathrm{MeV}$ and below $100 \mathrm{MeV}$, neutrons are produced by pseudo-deuteron mechanism. In this neutron production mechanism, photodisintegration of nucleon pairs within the nucleus takes place. Pseudo-deuterons are 
more energetic than GRNs. The cross section for this mechanism is an order of magnitude lower than the GRN peak.

\section{Photopion}

Photons of energy above the pion threshold ( $140 \mathrm{MeV})$ produce neutrons by photopion reaction. These neutrons are much higher in energy than those from GRN and pseudo deuterons. The cross section goes through a number of resonance peaks. At very high energy electron accelerators, these neutrons make the dominant contribution to the dose rate outside the massive concrete shielding. Only the neutrons with the highest energies are able to penetrate through the thick shielding. The lower energy portions of neutron spectra outside the shielding are generated by these high energy neutrons interacting in the outer layers of the shielding.

\subsection{Objective}

The objective of this work was to calculate the neutron arrival time distributions for common neutron detectors and compare the results with the measurements made outside the shielding of the FFTB beam dump. The T-values for FFTB spectrum have also been estimated for various neutron detectors from their calculated neutron arrival time distributions. The detectors used for this study were a moderated $\mathrm{BF}_{3}$ counter, an $\mathrm{AB}$ remmeter, and Bonner Sphere detectors (LiI sensor) of 2", 3", 5", 8", 10", and 12" diameter. 
The neutron arrival time distribution is a differential plot of the number of neutrons detected by the sensor versus their corresponding arrival time. In the moderating type detectors, there can be a substantial delay for neutrons to be detected after their incidence on the detector surface. The delay depends on the thickness of the moderator. The significant time delay does not result from the slowing down of the neutrons to thermal energies, but from the diffusion of the thermal neutrons in the moderator.

The investigation of the timing effect of the moderator thickness on the arrival of the neutrons is the primary focus of this study. The neutron arrival time distributions along with the energy response of the various detectors have been calculated using the MCNP4A Monte Carlo code (MCNP 1993). Measurements have been performedi with four detectors to record the number and arrival time of the neutrons. The neutron arrival time for the measurements (unlike that for the calculations) is referred to the time between the beam hitting the target and the neutron detected by the detector.

Since the neutron signal is roughly exponential in the arrival time distribution, the inverse of the slope of these curves gives the mean arrival time. Therefore, the estimation of the slope is important for the calculation of the dead time correction factor for moderating type detectors. 


\subsection{Summary}

Thus far, Chapter 1 has given an introduction to the dead time correction and its importance in pulsed radiation fields for moderating type detectors. The objective of the study has also been outlined.

Chapter 2 describes the geometry of the detectors used in this study, namely, the moderated $\mathrm{BF}_{3}$ detector, $\mathrm{AB}$ remmeter, and Bonner Sphere detectors. Section 2.2 provides general discussion of the MCNP code along with some details of the input files developed for this study. In Section 2.3, the tally summary of MCNP output file is discussed. Neutron sources defined for the MCNP calculations are described in Section 2.4 .

Chapter 3 describes the neutron arrival time measurements made at FFTB. The location of detectors is described in the Section 3.1. The associated electronics for the timing measurements is described in Section 3.2.

The results of the MCNP calculations are discussed in Section 4.1 of Chapter 4. Section 4.2 gives a comparison between the measurements and the calculations. Section 4.3 is the calculation of the T-values.

Section 5.1 of Chapter 5 is a summary of the study. Conclusions that can be drawn from the study are discussed in Section 5.2. Section 5.3 contains suggestions for further studies. 


\section{MONTE CARLO CALCULATIONS}

\subsection{Introduction}

The arrival time distributions of neutrons for various detectors are calculated using the latest version of the MCNP4A Monte Carlo Code (MCNP 1993). This chapter describes the basic concept of a Monte Carlo approach and introduces the way the MCNP was used for this study. Geometry of the detectors used in this work is discussed in detail in Section 2.2. Neutron sources (monoenergetic and spectra) used for the calculations are also presented.

The general-purpose Monte Carlo N-Particle (MCNP) code is used for calculating time-dependent continuous-energy transport of neutrons, photons, and/or electrons in three-dimensional geometry. The code provides a number of tally (the desired result) output options and various variance reduction techniques that improve the efficiency of the calculations.

When a neutron travels through a material, it interacts with the constituent atoms of that material. Whether the neutrons become absorbed or scattered depends on the neutron interaction cross section in that material. It cannot be predicted exactly how the particles will interact or how will they travel in the material before being scattered or captured. However, the probability of occurrence of one or the other process can be determined by the relevant cross sections. The cross sections can enable us to predict the distribution of flight distances (mean free path) that a large number of particles would have before the 
first interaction. This is done by random-walk analysis in the MCNP code. The randomwalk analysis is the statistical history for the life of each particle generated by computer using random numbers.

The MCNP code has extensive nuclear data and cross section libraries, which enables it to determine which process will occur at a particular time in the life of the particle. The life of a particle begins with its generation from the source and ends when it gets absorbed or scattered to move outside of the detector assembly. The code also takes into account how much energy is lost during each interaction and what is the new direction of the particle (if scattered) or how many neutrons created in any fission event.

A very large number of histories is evaluated to have a good statistics and to approach a more accurate result with a small uncertainty. Records of all the events in the life of the particle and the scored tally/tallies are tabulated in an output file (see Section 2.4).

In this work, the MCNP4A code is used to simulate transport of neutrons from the source to the sensor through different layers of materials, including the moderator. In the sensor, neutron captures are scored as a function of time. The description of the problem is given in an input file (see Section 2.3).

\footnotetext{
A set of numbers. which have no pattern. that are sampled uniformly between zero and one.
} 


\subsection{Geometry of detectors}

\subsubsection{Moderated $\mathrm{BF}_{3}$ detector}

A schematic drawing of a custom-made moderated $\mathrm{BF}_{3}$ detector is shown in Fig. 2.1. This detector is specially designed for the neutron fluence measurement. The moderated $\mathrm{BF}_{3}$ detector consists of a BF 3 proportional counter (Reuter Stokes RS-P1-1613: $31 \mathrm{~cm}$ long and $5 \mathrm{~cm}$ in diameter) with an attached preamplifier that is inserted into a cadmium (Cd)-covered polyethylene cylinder. In this device, boron trifluoride $\left(\mathrm{BF}_{3}\right)$ serves both as a target for conversion of neutron to secondary charged particles as well as a proportional gas. The gas is highly enriched in ${ }^{10} \mathrm{~B}$ to increase its efficiency. The absolute pressure in typical $\mathrm{BF}_{3}$ tube is $70 \mathrm{~cm} \mathrm{Hg}$. The whole assembly is encased in an aluminum cylinder.

The $\mathrm{BF}_{3}$ gas is a thermal neutron sensor. The thermal neutrons cross section for ${ }^{10} \mathrm{~B}(\mathrm{n}, \alpha)^{7} \mathrm{Li}$ reaction is 3840 bams. Neutrons that have been slowed down to a few hundreds of $\mathrm{eV}$ can also react with ${ }^{10} \mathrm{~B}$, however, the reaction cross section varies as $1 / \mathrm{v}$ and drops to about 100 barns at $100 \mathrm{eV}$. The reaction may be written as follows :

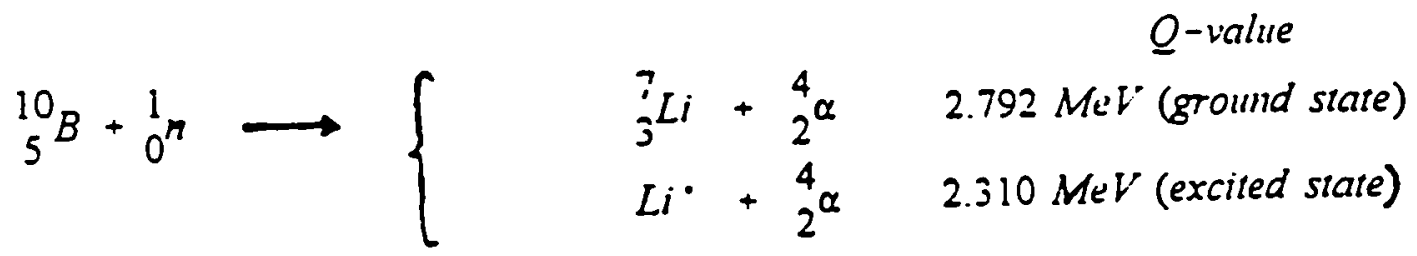




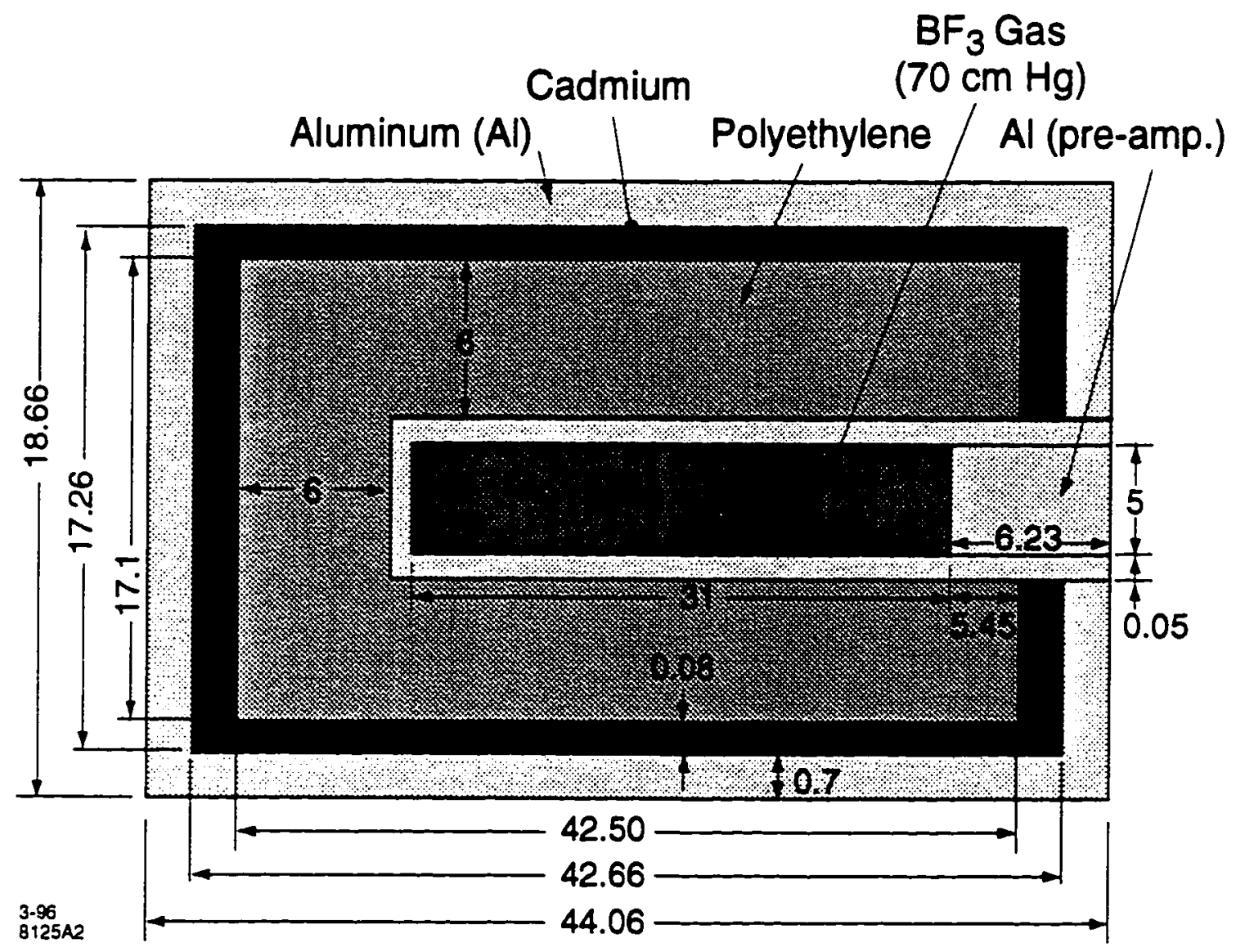

Fig. 2.1 Schematic representation of cross-section of the moderated $\mathrm{BF}_{3}$ detector. 
In either case (ground state or excited state), the Q-value of the reaction is very large compared to the energies of slow neutrons. The energy imparted to reaction products $\left({ }^{7} \mathrm{Li}\right.$ and $\alpha$ ) is the Q-value itself. Any information about the neutron energy is submerged in the much larger reaction energy.

The $\mathrm{BF}_{3}$ detector is relatively insensitive to gamma rays, because high energy photons lose small fractions of their energy in the detector. The resulting secondary electrons give rise to smaller pulses, whereas the ${ }^{7} \mathrm{Li}$ ion and alpha particles have relatively shorter ranges in the gases and deposit most of their energy (Q-value energy) to give rise to a larger pulse.

As mentioned in Chapter 1 , the purpose of the $\mathrm{BF}_{3}$ detector being inserted in the $\mathrm{Cd}$ covered polyethylene moderator is to use this detector for detecting fast neutrons. Most fast neutrons (above a few $\mathrm{MeV}$ ) incident on the cylindrical polyethylene moderator (a radial thickness of $6 \mathrm{~cm}$ and $42.5 \mathrm{~cm}$ long) are slowed down to thermal energy $(0.025 \mathrm{eV})$ before interacting with the $\mathrm{BF}_{3}$ gas. On the average, the elastic scattering of a fast neutron on hydrogen [in polyethylene $\left(\mathrm{C}_{2} \mathrm{H}_{4}\right)_{n}$ ] results in the loss of half of the initial neutron energy.

The polyethylene is covered with a $0.08 \mathrm{~cm}$ thick layer of cadmium (Cd) to ensure that only epithermal neutrons enter the moderator. Since $\mathrm{Cd}$ has a very high thermal neutron capture cross section of 3620 barn, it absorbs essentially all the incident thermal neutrons. Hence, the moderated $\mathrm{BF}_{3}$ detector responds only to the incident neutrons with energies greater than the Cd cut-off $(0.4 \mathrm{eV})$. 


\subsubsection{Anderson-Braun $(\mathrm{AB})$ remmeter}

A schematic drawing of an Anderson-Braun (AB) remmeter is shown in Fig. 2.2a. It has a $\mathrm{BF}_{3}$ proportional counter as the thermal neutron sensor. The detection technique works the same way as that for the moderated $\mathrm{BF}_{3}$ detector. The $\mathrm{BF}_{3}$ tube in this detector is only one third of the size of the one in the moderated $\mathrm{BF}_{3}$ detector. Since it is designed to measure dose equivalent rate, it is called a remmeter and the structure of its moderator is more complicated. The total thickness of the polyethylene is larger $(6.5+1.6=8.1 \mathrm{~cm})$ than that of the moderated $\mathrm{BF}_{3}$ detector.

Figure $2.2 \mathrm{~b}$ shows the $(\mathrm{AB})$ remmeter and its components. $\mathrm{A} 10.8 \mathrm{~cm}$ long and 3.4 $\mathrm{cm}$ in diameter aluminum tube is filled with $\mathrm{BF}_{3}$ gas. The gas has a pressure of $70 \mathrm{~cm} \mathrm{Hg}$. The $\mathrm{BF}_{3}$ tube is surrounded by an inner polyethylene $(15 \mathrm{~cm}$ long and $1.6 \mathrm{~cm}$ radial thickness), covered with a $0.5 \mathrm{~cm}$ thick boron plastic cylinder, and an outer polyethylene moderator which is $24.4 \mathrm{~cm}$ in length and of $6.5 \mathrm{~cm}$ radial thickness.

The boron plastic is boron doped synthetic rubber and has a number of holes in its lateral, front, and end surfaces. The holes are $10 \mathrm{~mm}$ in diameter. They cover $22 \%$ of the total boron plastic area. The purpose of the boron plastic is to capture some of the thermal and epithermal neutrons. This moderator design simulates an energy response which is close to the fluence to dose equivalent conversion factor curve for fast neutrons (ICRP 1973). 


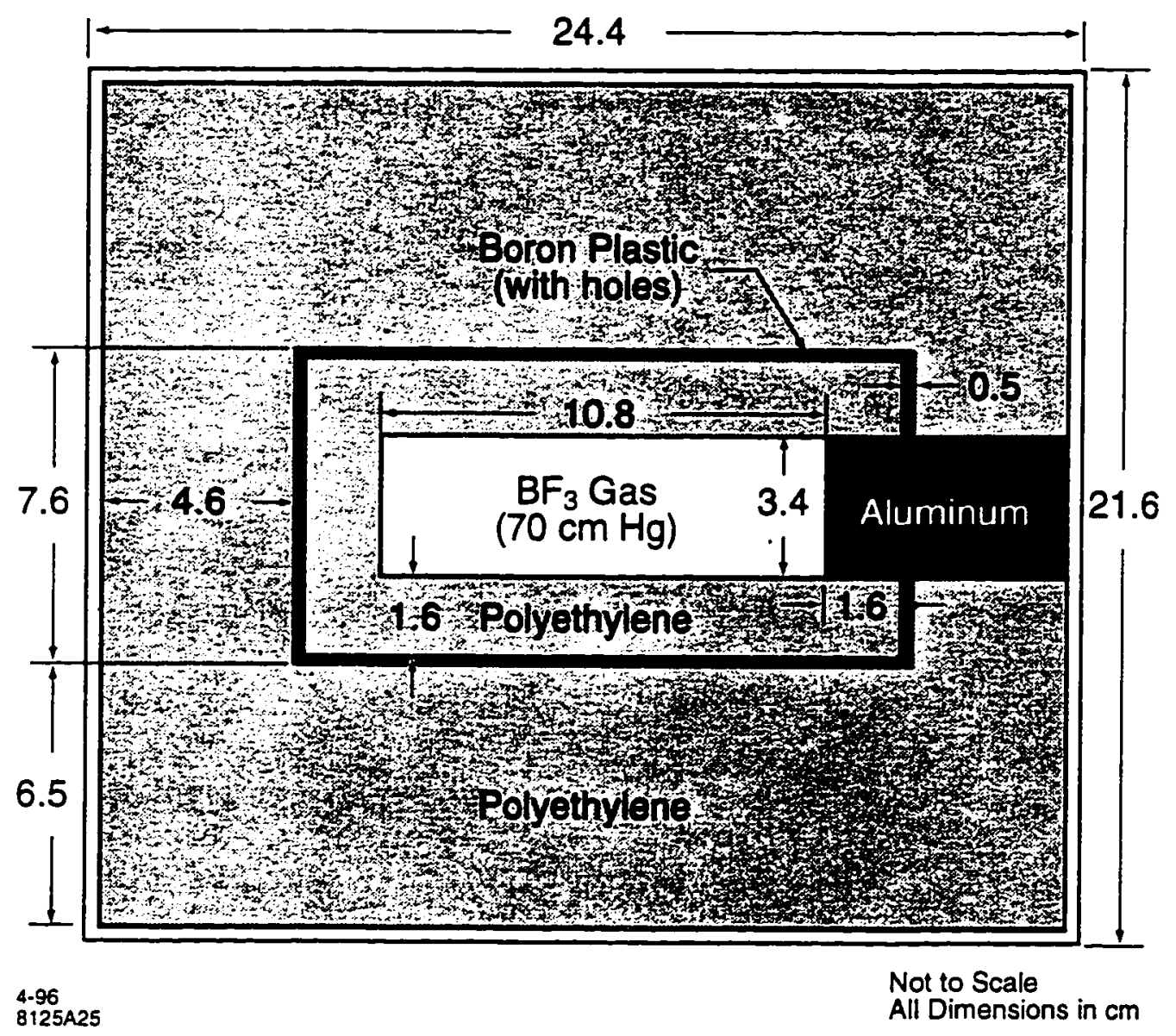

Fig. 2.2a Schematic representation of cross-section of the Anderson-Braun remmeter. 


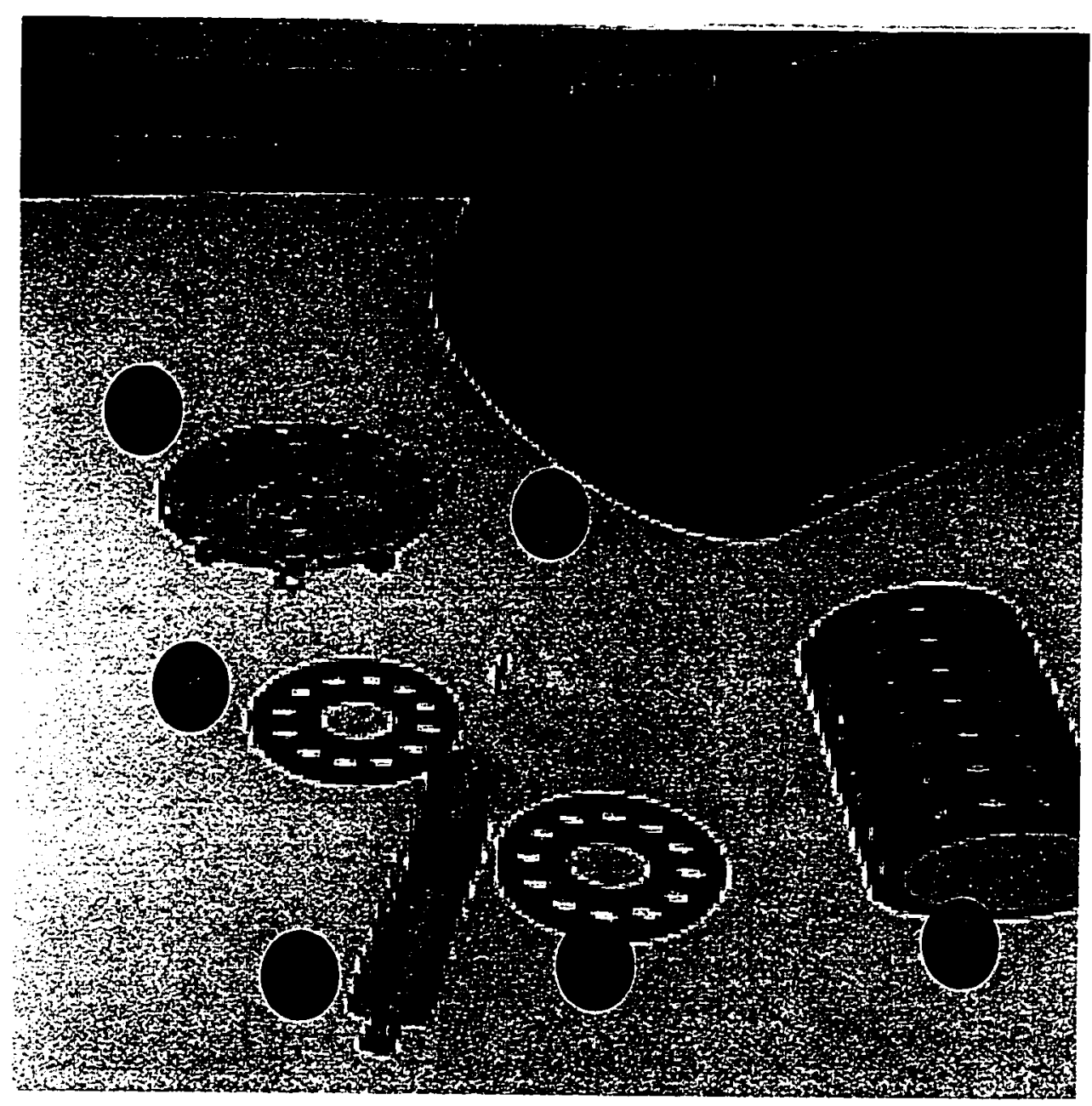

Fig 2.2b The Anderson-Braun (AB) remmeter and its components: 1) The main body of this detector is the large polyethylene cylinder in the background. 2) The Reuter Stokes $\mathrm{BF}_{3}$ tube model RS-P1-0802-104. 3) Smaller polyethylene cylinder with boron enriched plastic cover. 4) Boron enriched plastic end inserts. 5) Aluminum end cap with high voltage connector. 
Due to its pseudo-spherical body $(24.2 \mathrm{~cm} \times 21.6 \mathrm{~cm})$, the response of an $A B$ remmeter is similar for neutrons incident from the side and that from the end (Liu 1991). During this work, the detector was placed laterally relative to the source direction.

\subsubsection{Bonner Sphere detector}

A schematic drawing of a Bonner Sphere detector probe is shown in Fig. 2.3a. The detector has a $4 \mathrm{~mm} \times 4 \mathrm{~mm}$ enriched ${ }^{6} \mathrm{LiI}(\mathrm{Eu})$ crystal as the thermal neutron sensor. The ${ }^{6} \mathrm{Lil}$ (a scintillator) is coupled to photomultiplier tube (PMT) by a $10 \mathrm{~cm}$ long Plexiglas light guide. The scintillator crystal, light guide, and photomultiplier are placed in an aluminum housing; together, they constitute the detector probe. The Bonner Sphere detector probe is placed inside the polyethylene sphere so that crystal lies at the center of the sphere. This is called the detector assembly. Example of placement of sphere over detector probe is shown schematically in Fig. 2.3b.

Neutrons arrive at the center of the moderator as thermal neutrons and interact with the scintillator via ${ }^{6} \mathrm{Li}(\mathrm{n}, \alpha)^{3} \mathrm{H}$ reaction. The reaction produces a total of $4.79 \mathrm{MeV} \alpha$ and ${ }^{3} \mathrm{H}$ particles that are stopped in the ${ }^{6} \mathrm{LiI}(\mathrm{Eu})$ crystal. The crystal then produces light pulses. The light pulses guided through the light guide reach PMT and are converted to electronic signals that are recorded with the associated counting system. 


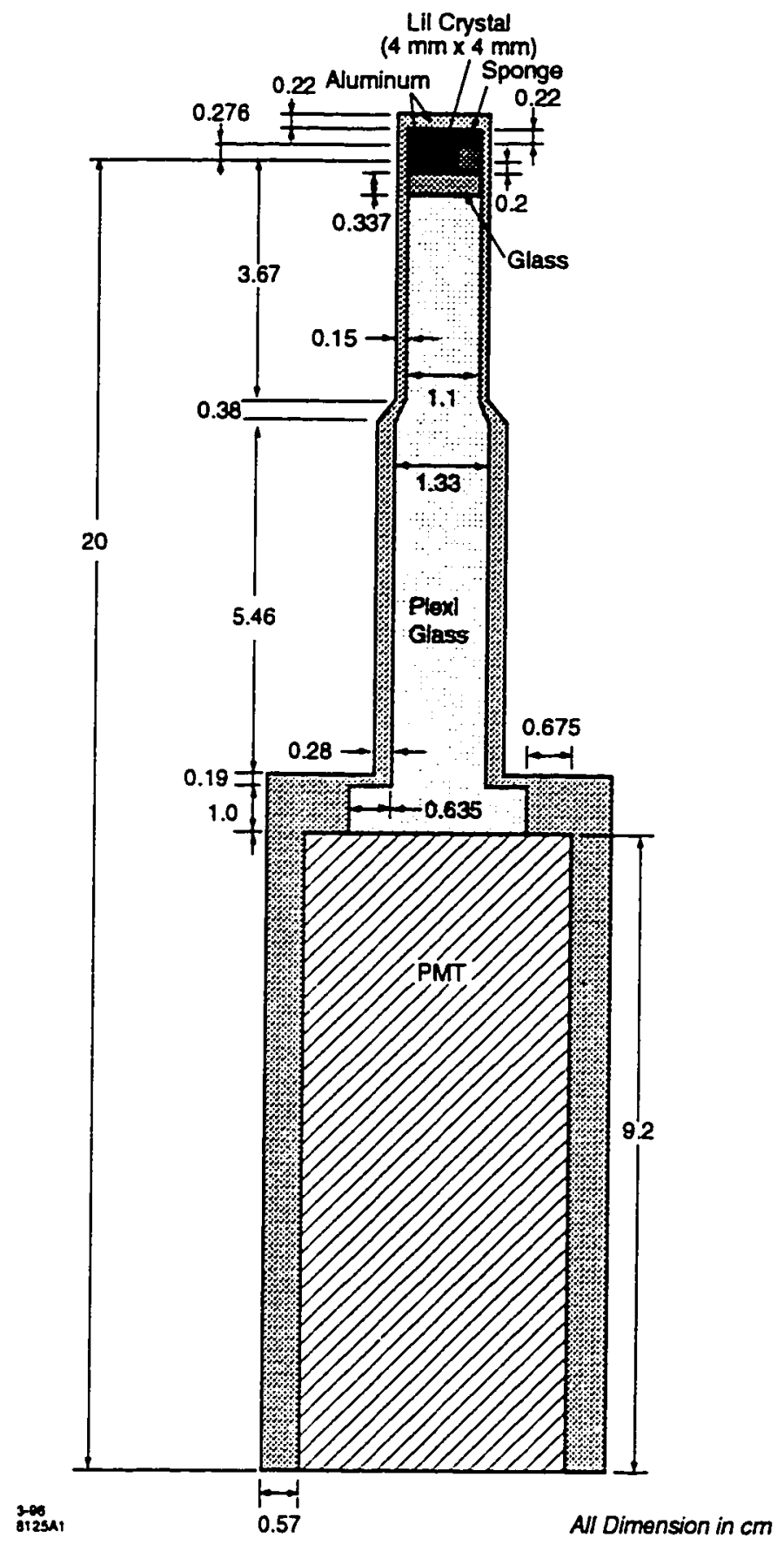

Fig. 2.3a Schematic representation of cross-section of the Bonner Sphere detector probe. 


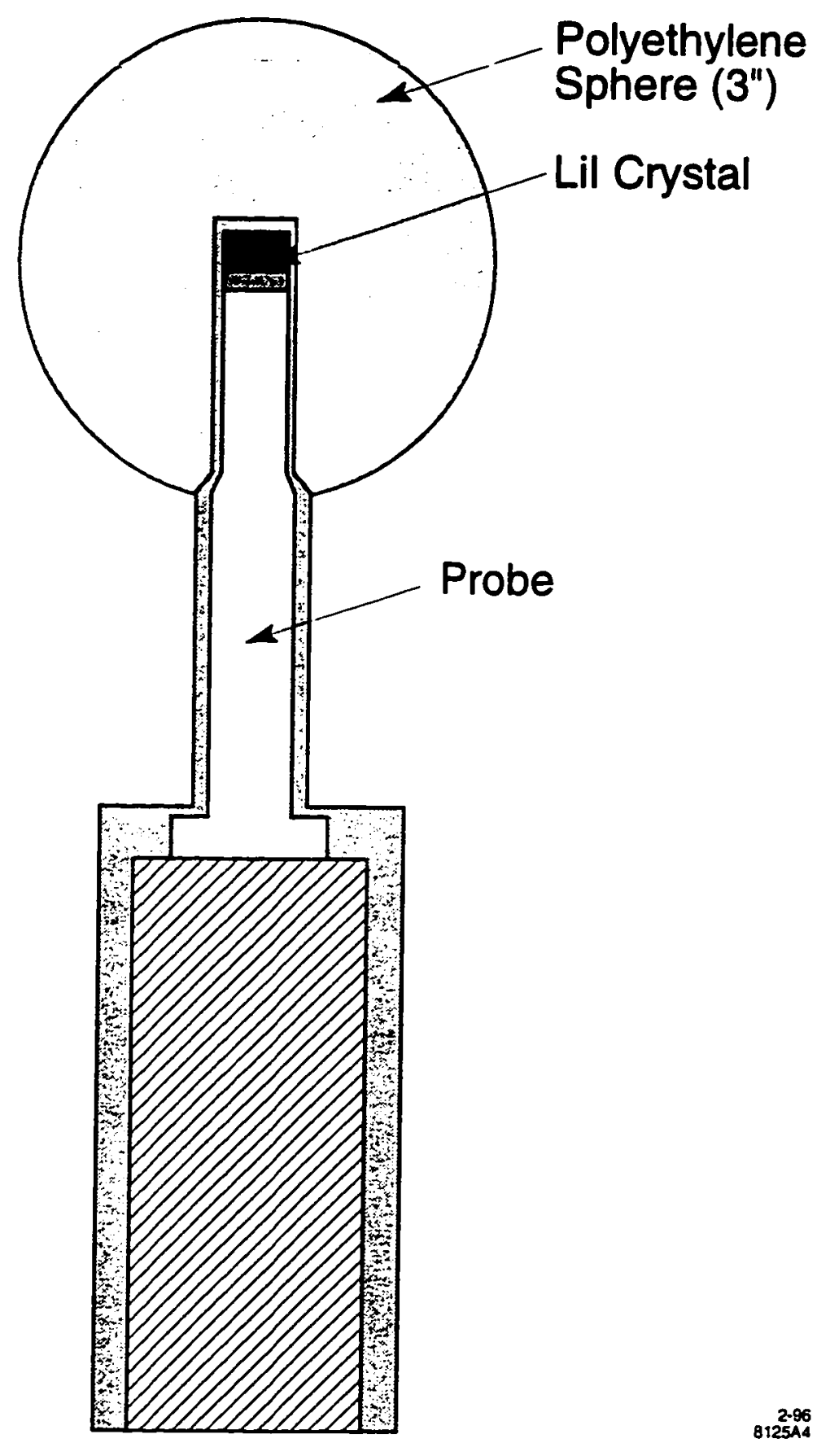

Fig. 2.3b Schematic representation of cross-section of the Bonner Sphere detector assembly. 
The response of this instrument as a function of neutron energy varies substantially with the size of the moderator (Knoll 1989). Therefore, polyethylene spheres of various diameters are used to detect neutrons in different energy range. The different thickness of the moderator also affects the neutron arrival time distributions. Being spherical in shape, the angular response of Bonner Spheres detector is almost perfectly isotropic. In this work, the detector was placed in such a position that the probe axis was perpendicular to the incident neutron source.

\subsection{MCNP input file}

This section briefly describes an MCNP input file. The input file includes the problem geometry, source definition, tallies, material specification, physics of the problem, histories to be run, and the tables desired to be printed in the output file. The MCNP input file consists of these sections: Title card, Cell cards, Surface cards, and Data cards.

Appendix $\mathrm{A} 1$ is $\mathrm{MCNP}$ input file of the moderated $\mathrm{BF}_{3}$ detector for monoenergetic neutrons. The geometry of the detector is simulated in details. The $\mathrm{BF}_{3}$ gas has a density of $0.002567 \mathrm{~g} / \mathrm{cm}^{3}$, with the boron enriched $96 \%{ }^{10} \mathrm{~B}$. Density of the polyethylene moderator is $0.95 \mathrm{~g} / \mathrm{cm}^{3}$.

The code models a $20-\mathrm{MeV}$ neutron source incident on the surface perpendicular to the axis of the detector and uniformly distributed over the detector surface. The average neutron flux is scored in the sensor $\left(\mathrm{BF}_{3}\right.$ gas) for the reaction $(n, \alpha)$ that is indicated by a

\footnotetext{
The word "card" is used throughout this chapter to describe a single line output up to 80 characters.
} 
reaction number 107 . The results are multiplied by a constant 0.024 (atomic density of $\mathrm{BF}_{3}$ ) to include the normalization of "per $\mathrm{cm}^{3 "}$ (MCNP 1993). The tally is separated into 50 time bins from $0 \mu \mathrm{s}$ to $500 \mu \mathrm{s}$. Hence, the number of $(\mathrm{n}, \alpha)$ reactions per $\mathrm{cm}^{3}$ in $\mathrm{BF}_{3}$ are calculated in each time bin.

Appendix $A 2$ is MCNP input file of the moderated $\mathrm{BF}_{3}$ detector for GRNs. The GRN spectrum is generated by the MCNP using the relation $P(E)=C E \exp (-E / a)$ for which $C$ is a constant and the parameter ' $a$ ' is set as $1 \mathrm{MeV}$ (MCNP 1993). The MCNP calculations for FFTB spectrum are done by constructing an input file in which measured FFTB neutron spectrum is entered as the cumulative source probabilities for various energy bins (see Appendix A3).

The input files for other detectors were developed in a similar manner by defining appropriate geometry and material specification. Also, the appropriate tally multiplicative constant and reaction number are specified for each detector. Appendices B1, B2, and B3 are the MCNP input files of the $A B$ remmeter for monoenergetic 1-MeV neutrons, GRNs, and the measured FFTB neutron spectrum, respectively. The reaction number for the remmeter remains the same as 107 , because it has the same sensor as that of $B F_{3}$ detector. The tally is separated in 24 time bins from $0 \mu \mathrm{s}$ to $200 \mu \mathrm{s}$. To simplify the geometry input, instead of defining holes in geometry, a reduced material density is entered on the cell card. The density of the boron plastic in the remmeter is reduced directly by $22 \%$ (from 1.0 to $\left.0.78 \mathrm{~g} / \mathrm{cm}^{3}\right)$. 
For the Bonner Sphere calculations, a reaction number 105 is entered which corresponds to ${ }^{7} \mathrm{Li}(\mathrm{n}, \alpha)^{3} \mathrm{H}$ reaction in LiI crystal. The multiplicative constant in this case is 0.11733 , which is the atom density of LiI. The neutron source is positioned on the plane normal to the probe axis and is uniformly distributed. Appendices $\mathrm{C} 1, \mathrm{C} 2$, and $\mathrm{C} 3$ are the examples of the MCNP simulation of monoenergetic I-MeV neutrons, GRNs, and the measured FFTB neutron spectrum, respectively, for various Bonner Spheres. The polyethylene spheres used for the calculations are of $2^{\prime \prime}, 3^{\prime \prime}, 5^{\prime \prime}, 8^{\prime \prime}, 10^{\prime \prime}$, and 12" diameter. The geometry of detector assembly was defined for each sphere in a separate input file.

\subsection{MCNP output file}

When a problem runs successfully, MCNP loads significant amounts of information in the output file. The tallies are printed in the end portion of the output file as the problem summary. The output file includes the following information:

- Echo of input file.

- Description of cell densities and masses.

- Material and cross section information.

- Neutron creation and loss summary table.

- Problem summary.

- Error analysis table.

A portion of output file of the moderated $\mathrm{BF}_{3}$ detector for monoenergetic 1-MeV neutrons is shown in Appendix D. It includes the averaged neutron fluence per source 
neutron for the corresponding time bin with the statistical uncertainties in each time bin. At the end of the tally summary, the total number of scored neutrons in all time bins (1.51896E-05) is also listed. It corresponds to the total area in the arrival time distribution curve. This is the response in counts per incident neutron.

The MCNP code also provides warning messages to inform the user about possible mistakes. The problem, however, will run with the warning messages. All warning messages from an MCNP calculation are examined and understood to be certain that there is no mistake in the problem specification.

\subsection{Neutron sources}

For the calculation purpose, two types of spectra and various monoenergetic neutrons were defined on the source card of separate input files for each detector. The monoenergetic sources were in the $0.1 \mathrm{eV}$ to $20 \mathrm{MeV}$ energy range.

A GRN spectrum was generated by the MCNP4A code itself by using a value of 1 $\mathrm{MeV}$ for the parameter ' $\mathrm{a}$ ' in the evaporation spectrum formula $P(E)=C E \exp (-E / a)$. The spectrum is shown in Fig. 2.4. It has a neutron fluence peak at $1 \mathrm{MeV}$ and the intensity drops to minimum at $10 \mathrm{MeV}$. The spectrum has a small component of low energy neutrons.

The other neutron spectrum was the measured FFTB spectrum (Fig. 2.5) in a 'hot spot' location (Thai 1996), at which the measurements in this work were made. The FFTB spectrum exhibits a two-peak structure. 


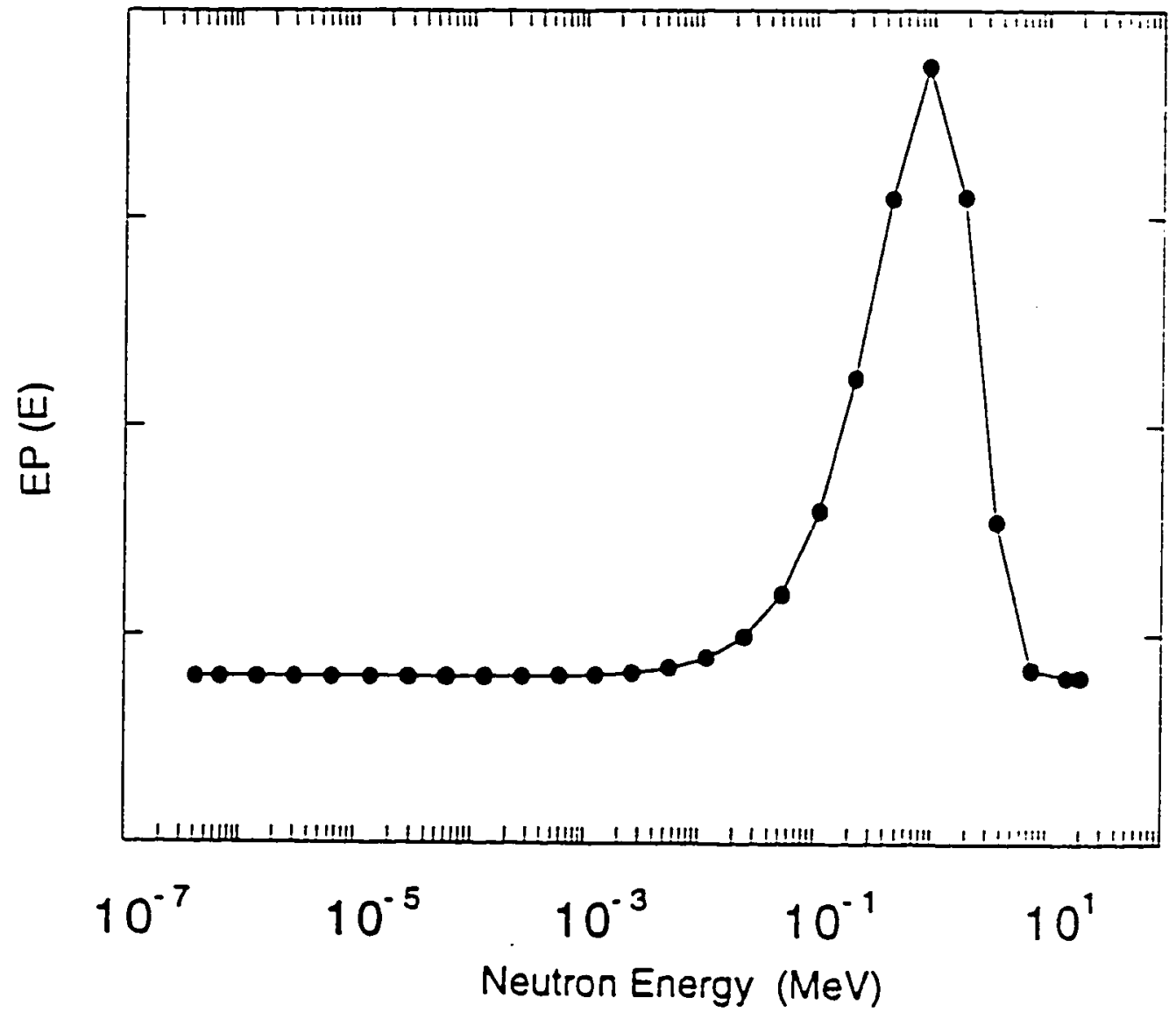

Fig. 2.4 MCNP-calculated GRN spectrum using the parameter ' $a$ ' $=1 \mathrm{MeV}$ in the formula $P(E)=C E \exp (-E / a)$. Notice that the peak is at $1 \mathrm{MeV}$. 


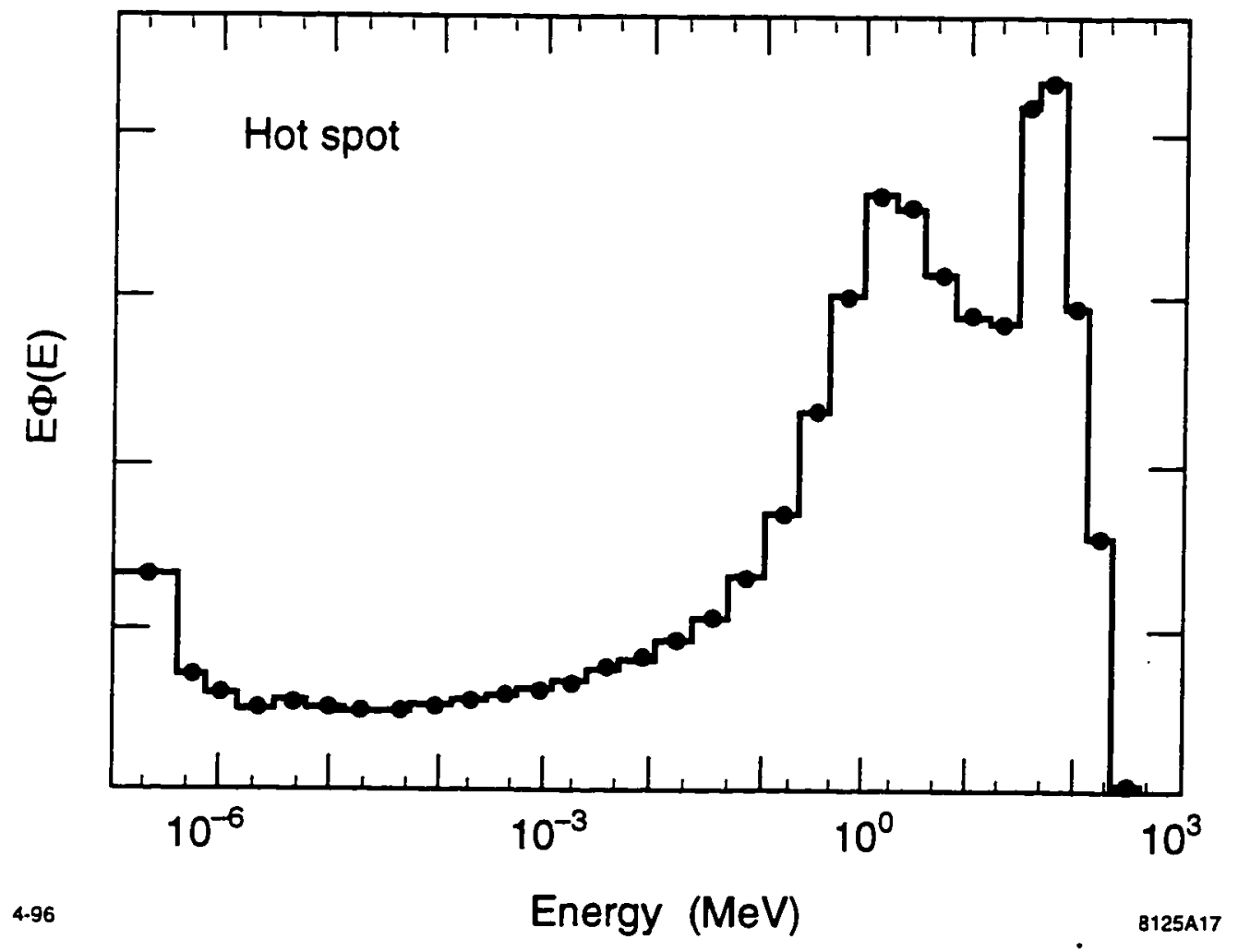

Fig. 2.5 The measured neutron spectrum for FFTB-'hot spot' location. The spectrum has a two peak structure. 
The one at higher neutron energy $(\sim 100 \mathrm{MeV})$ results from the slowed-down photopion neutrons. These are the neutrons produced by photopion reaction and have highest energies of all, which could penetrate the thick shielding. The lower energy peak at 1 $\mathrm{MeV}$ is generated by the high energy neutrons in the outer layers of shielding.

Both, the GRN and FFTB spectra were simulated up to a $20 \mathrm{MeV}$ neutron energy for the MCNP calculations. This is the upper energy limit in the MCNP code. Therefore, for the measured FFTB spectrum, the neutrons above $20 \mathrm{MeV}$ are not included in the calculations. Due to the low sensitivity of the detector to neutrons above $20 \mathrm{MeV}$, the calculations without the inclusion of neutrons above $20 \mathrm{MeV}$ are acceptable. 


\section{MEASUREMENTS}

\section{3.i introduction}

The locations of the various detectors at which the measurements were performed are described in this chapter. The associated electronics and the procedure of collecting data are also discussed.

The measurements for the purpose of our work were possible only when the beam was available for certain high energy physics experimental research. The Final Focus Test Beam (FFTB) facility focuses the $46.7 \mathrm{GeV}$ electron beam to an extremely small size needed for the NLC (Next Linear Collider) project. The beam is then sent to a beam dump which is a metallic cylinder of iron and aluminum. The measurements were performed outside the shielding of the FFTB beam dump on the following days: $12 / 14 / 95$, $12 / 19 / 95$ and $12 / 20 / 95$. During the measurements the electron beam parameters were as follows; beam energy $=46.7 \mathrm{GeV}$, beam current $=0.7 \times 10^{10} \mathrm{e} /$ pulse, pulse repetition rate $=30 \mathrm{~Hz}$, and the average beam power $=1.56 \mathrm{~kW}$.

The schematic drawing of the location of the detectors outside the FFTB beam dump shielding is shown in Fig. 3. Ia. The beam dump is surrounded by 2 to 4 feet of iron and 4 to 5 feet of concrete shielding to reduce the dose rates to acceptable levels. A 22-m-long iron block is placed in the forward direction with respect to the beam to shield against the muons

\footnotetext{
Muons $\left(\mu^{-}\right)$are produced in the forvard direction. through pair production. the way electrons $\left(e^{7}\right)$ are.
} 


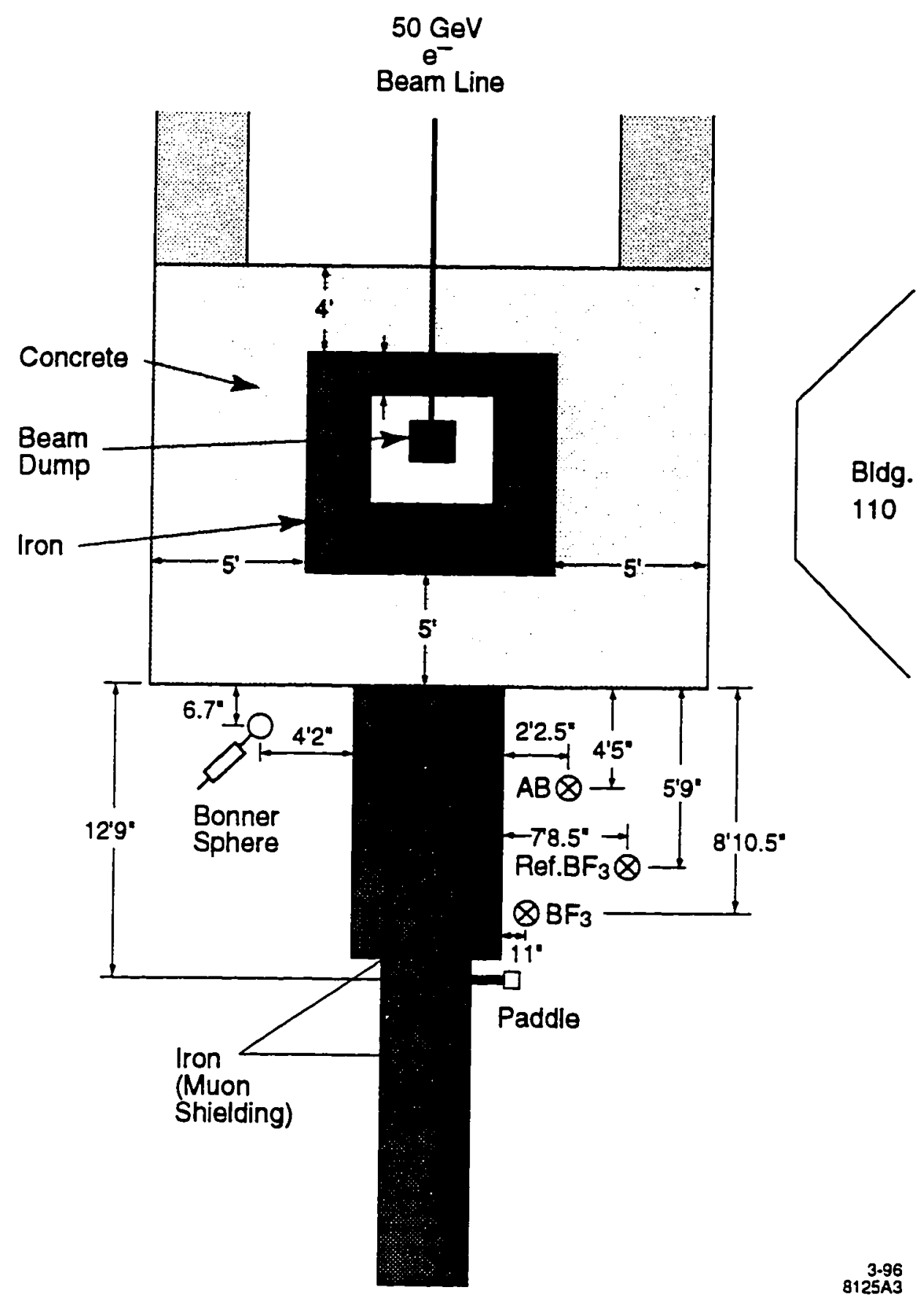

Fig. 3.1a Schematic drawing of the locations of all the detectors for the measurements outside the shielding of FFTB beam dump. 
Figure $3.1 \mathrm{~b}$ shows the placement of two moderated $\mathrm{BF}_{3}$ detectors, a remmeter and a paddle (the paddle shaped scintillation detector) outside the shielding of the FFTB dump. The paddle was placed on the muon shielding to detect the muons which indicates the presence of the electron beam. The upper right corner of the Fig. 3.1b shows the Bldg. 110 (close to shielding wall) which housed the associated counting electronics. Fig $3.1 \mathrm{c}$ shows the location of the Bonner Sphere detector (with 2" polyethylene sphere on the detector probe) on the other side of muon shielding, close to the concrete wall.

All the measurements were made on the ground outside the shielding wall, in the forward direction with respect to the beam. These locations have been found to have the highest dose rates and are called the FFTB 'hot spot' (Thai 1996). The data were collected simultaneously for all detectors at these locations. The data represent the number of neutrons (detected by the sensor of the detector) with respect to their arrival time. The arrival time in the measurements is the period between the instant when the beam hits the target and when the sensor detects the neutrons.

\subsection{Electronics}

The schematic of the associated electronics for the timing measurements is shown in Fig. 3.2. As shown in this figure, the associated counting electronics for each of the detectors basically consists of a high voltage power supply, an amplifier, and two counters. The Bonner Sphere system has its own high voltage power supply and 


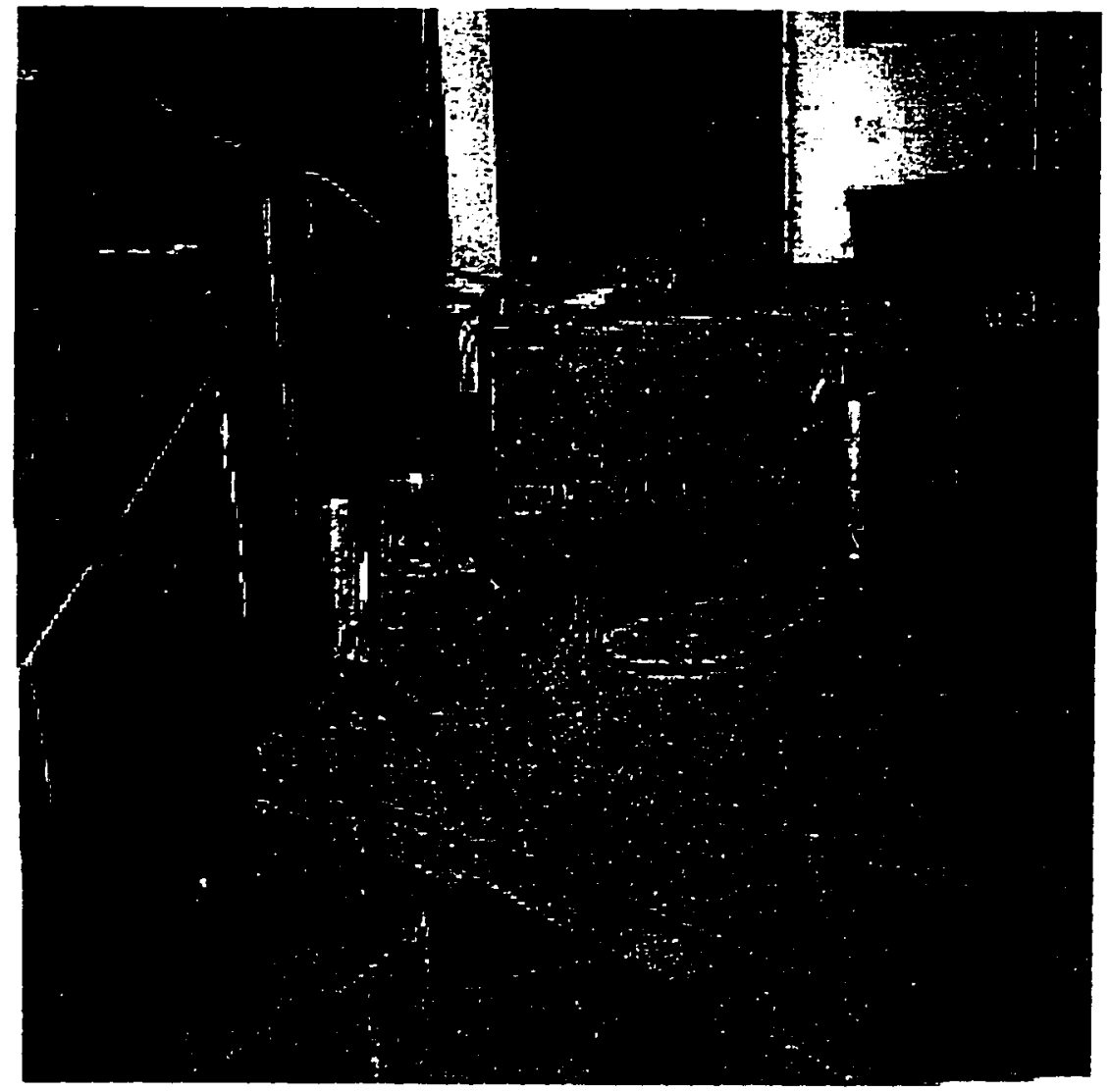

Fig.3.1b The location of the detectors relative to the FFTB concrete shielding (a) and muon shielding (b):

1) a moderated $\mathrm{BF}_{3}$ detector, 2) a moderated $\mathrm{BF}_{3}$ reference detector, 3) an $\mathrm{AB}$ remmeter, and 4) a paddle detector 


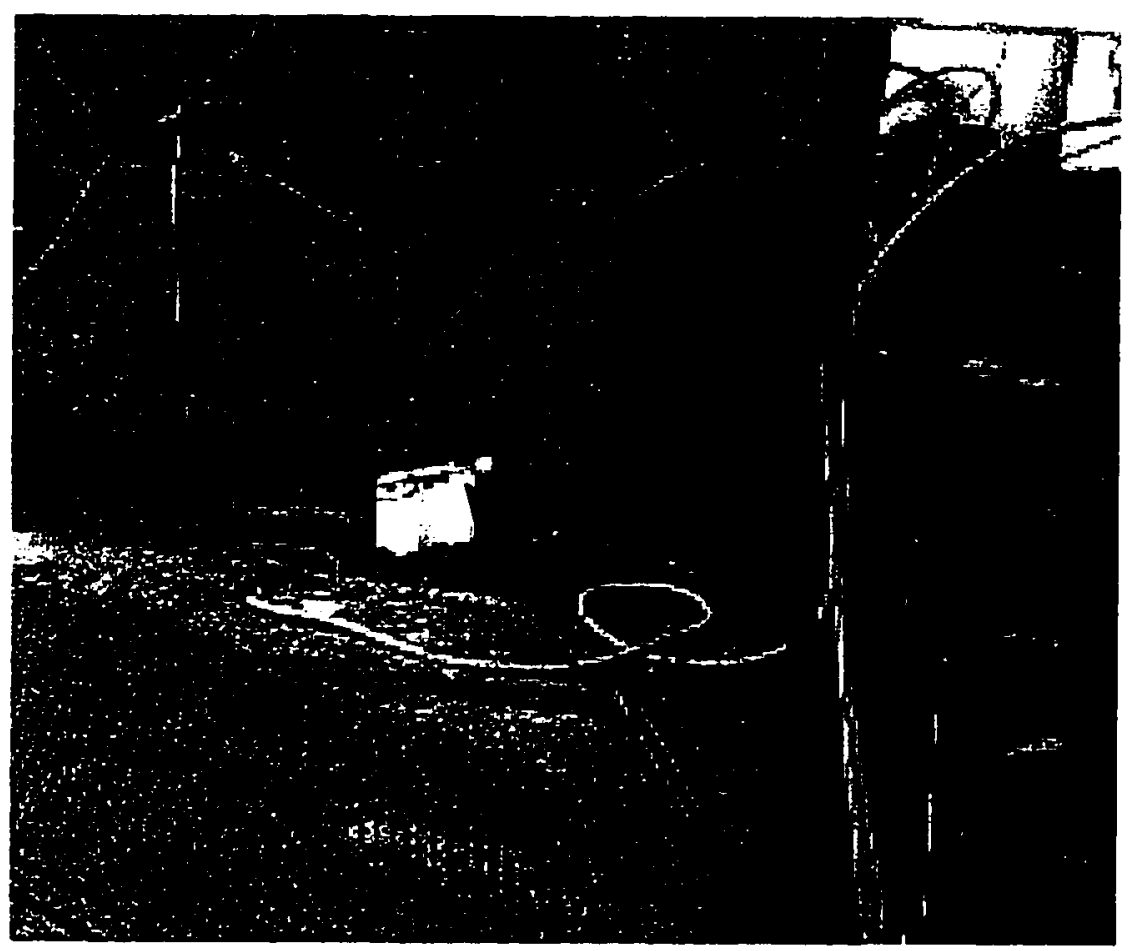

Fig.3.1c The location of the Bonner Sphere detectors (1) relative to the FFTB dump concrete shielding (2) and the iron muon shielding (3) 

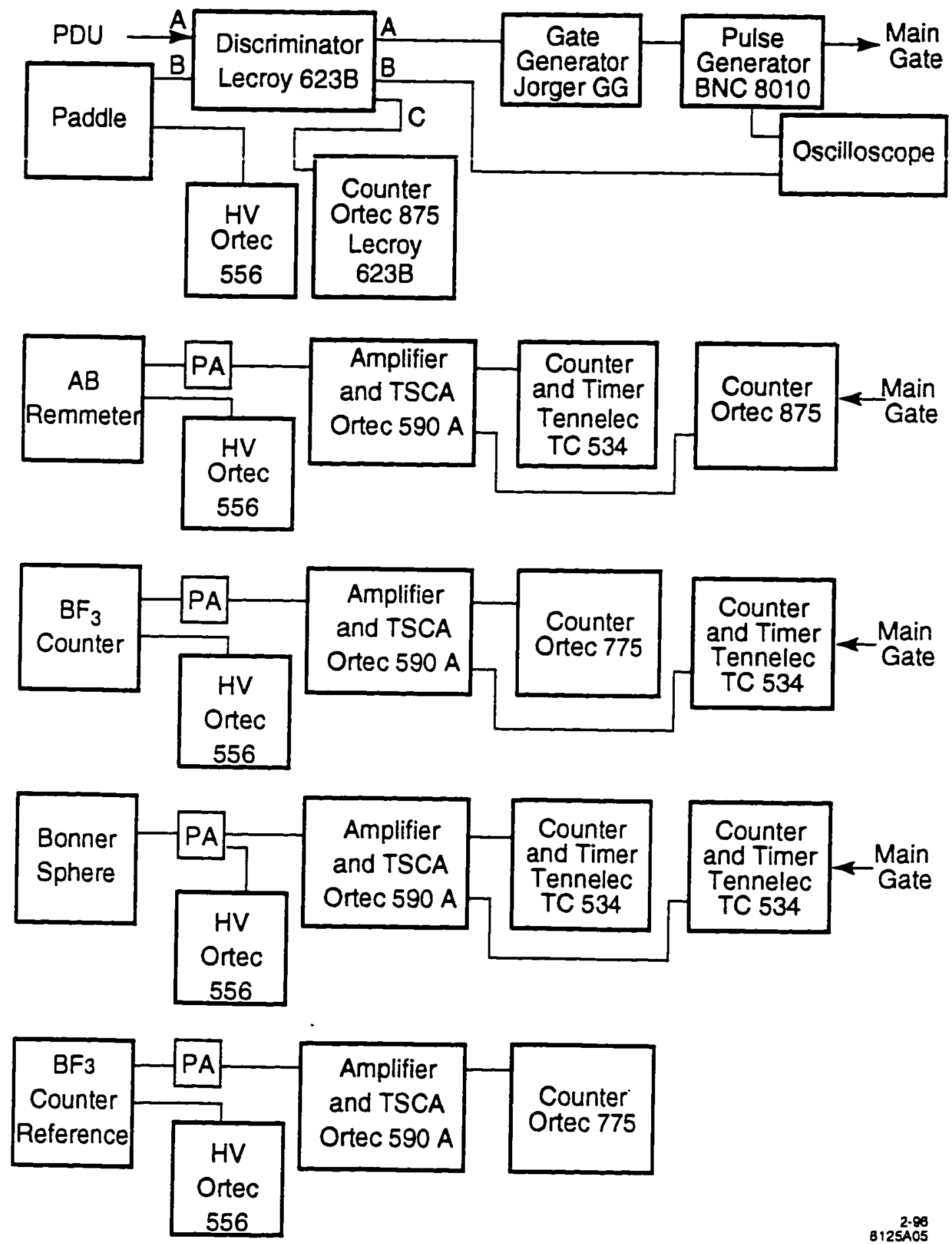

2.96
$8125 A 05$

Fig. 3.2 Schematic drawing of the electronics setup for the counting measurements made outside the shielding of FFTB beam dump. 
amplifier built in the ORTEC "Nomad" multichannel analyzer as a part of its counting system. The Bonner Sphere system was operated with the following parameters: high voltage $=+600 \mathrm{~V}$, coarse gain $=100$, and fine gain $=2.0$. The remmeter was set at high voltage $=+1800 \mathrm{~V}$, coarse gain $=50$ and fine gain $=1.5$. The settings for two moderated $\mathrm{BF}_{3}$ were as follows: high voltage $=+1800 \mathrm{~V}$, coarse gain $=50$, fine gain $=1.5$. The paddle detector was operated with a high voltage supply of $-1200 \mathrm{~V}$.

The presence of the beam was detected by the paddle signal. The analog pulse from the paddle was converted into digital pulse with a discriminator and counted. The leading edge of this digital pulse indicates the time of occurrence of an input analog pulse (i.e. electron beam). This signal acts as a trigger to the gate generator and a gate pulse is generated. Then the gate pulse is used to trigger a pulse generator. The output signal from the pulse generator constitutes the main gate. The main gate is fed to one of the two counters of each detector to record the gated signal. The other counter records the ungated signal.

The combination of the pulse generator and the gate generator enables the gate to be opened at desired time width. In the absence of the beam, pulses from the $\mathrm{PDU}^{+}$(see fig. 3.2) allowed us to set the timing of the gate generating circuit within the resolution of electronics system. In the presence of the beam, the PDU was adjusted to coincide with the beam signal.

\footnotetext{
The gate pulse is a standard logic pulse whose width is adjustable. 'PDU stands for Programmable Delay Unit and is used to synchronize the timing signal with the beam signal.
} 


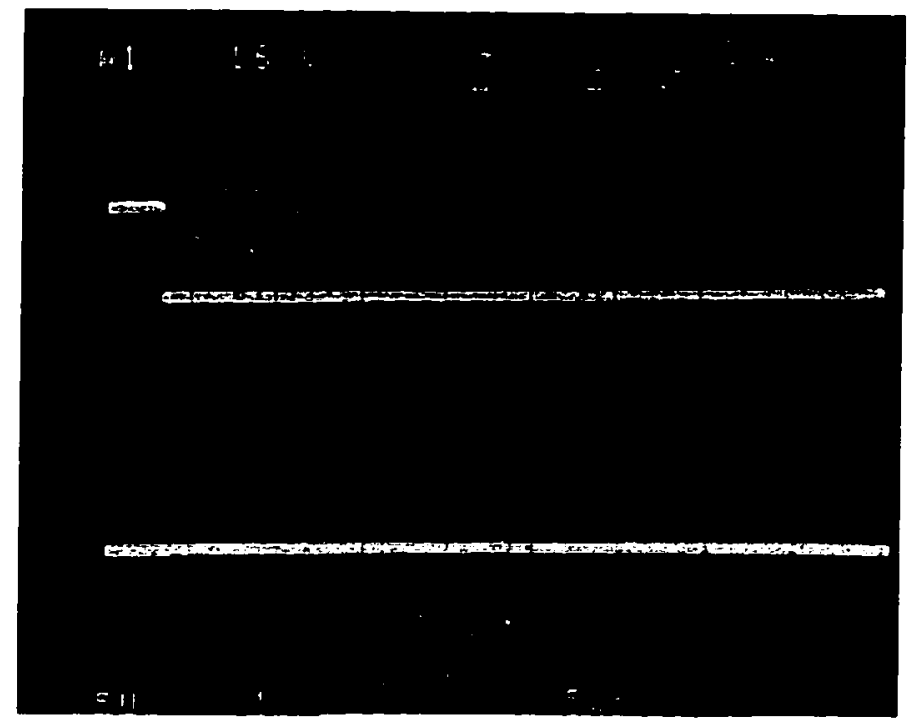

Fig. 3.3a The photograph of the oscilloscope screen displaying that the position of the gate (1) is ahead of the signal from the paddle (2). At this position of the gate, gated counters do not detect any neutrons. 

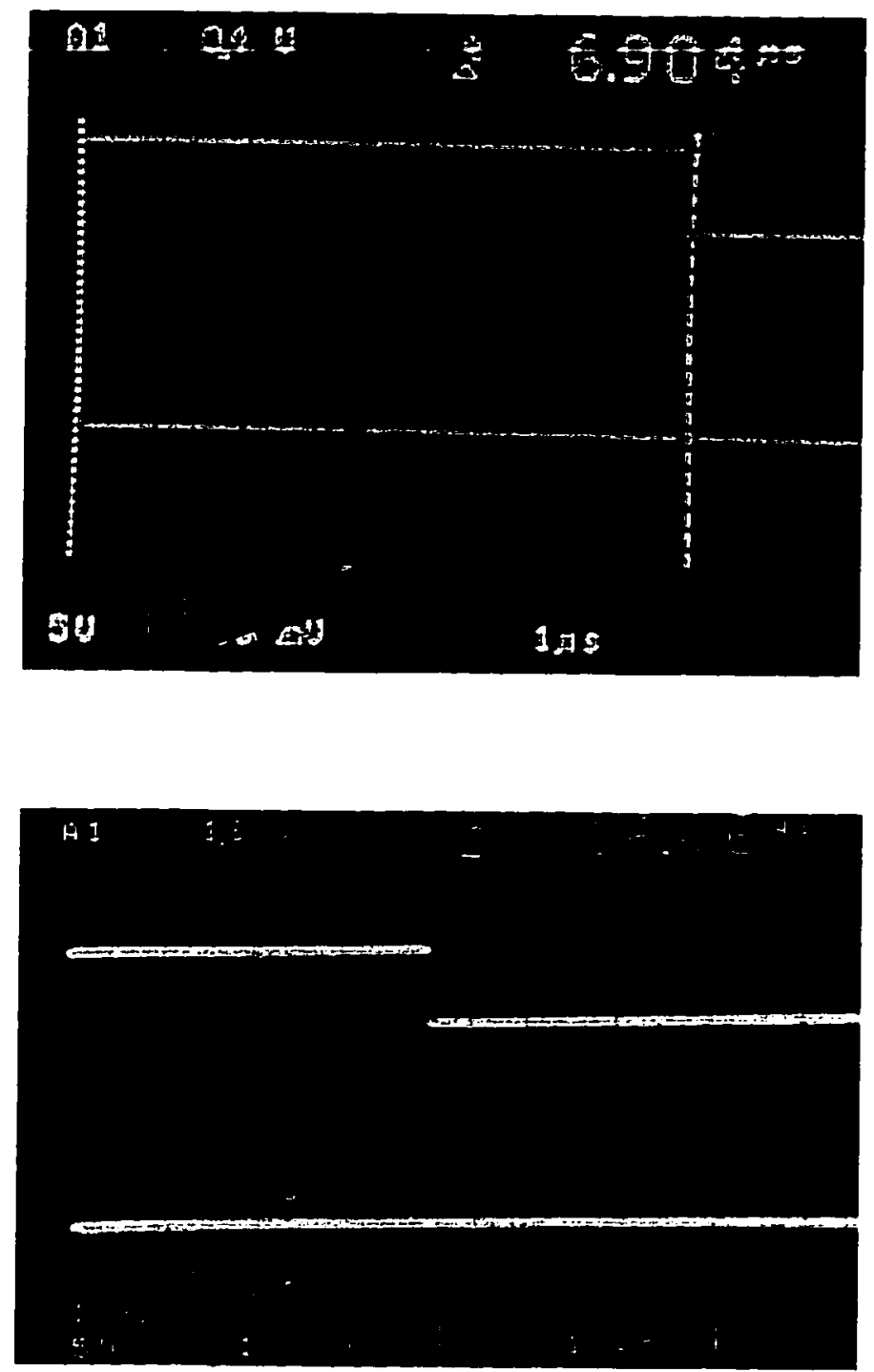

Figs. 3.3b The photograph of the oscilloscope screen displaying the position of the gate (1) and the signal from the paddle (2).

(top) The width of the gate is $6.904 \mu \mathrm{s}$ and the signal is at $3.8 \mu \mathrm{s}$ before the gate ends. (bottom) The gate width is shifted to $40 \mu \mathrm{s}$ and the signal is at $16.46 \mu \mathrm{s}$ before the gate ends. 
Figures $3.3 \mathrm{a}$ and $3.3 \mathrm{~b}$ show the movement of the gate as seen on the oscilloscope screen. In Fig. 3.3a the gate pulse is arriving earlier in time by $23.48 \mu$ s than the paddle signal, however, the gate width is only $5 \mu \mathrm{s}$. At this position of the gate, the detectors do not 'see' any neutrons at gated counters. The width of the gate pulse is then adjusted, so that the gate is open when neutrons arrive. Figure $3.3 \mathrm{~b}$ show the different widths of the gate relative to the beam signal.

The gate width was increased in appropriate steps starting from the $2 \mu \mathrm{s} \mathrm{time} \mathrm{bin.} \mathrm{In}$ each time bin, the gated signal was recorded for every detector except for the reference moderated $\mathrm{BF}_{3}$ detector. The ungated signal was also recorded for all the detectors in the same setup.

The gated signal in each time bin represents the cumulative number of neutrons detected by the sensor within that time. The ungated signal refers to the total number of the neutrons detected by the sensor over all time bins. The gated and ungated signal for each detector were normalized to the reference moderated $\mathrm{BF}_{3}$ detector signal. These data were used to generate neutron arrival time distribution, which is a differential plot of the number of the neutrons as a function of their arrival time. The uncertainty in the differential representation of the data was large, and therefore, the integral representation of the data (ratio of gated to ungated signal) was used for the analysis.

The timing measurements for the moderated $\mathrm{BF}_{3}$ detector were also made previously (Rokni 1996) and had better statistics than the present work. The data taken previously were used for the analysis in this work. 


\section{RESULTS AND DISCUSSION}

\subsection{Calculations}

\subsubsection{Moderated $\mathrm{BF}_{3}$ detector results}

The results of the MCNP calculations for the moderated $\mathrm{BF}_{3}$ detector are given in Figs. 4. la, b, c, and d. The curves shown in these figures are called the neutron arrival time distributions. Figs. 4.1a, b, and c are the MCNP simulations for monoenergetic neutrons incident on the surface of the detector. Figure 4. Id shows the comparison of the arrival time distributions of $1 \mathrm{MeV}$ neutrons with those of GRN and FFTB spectrum.

The data used to generate these plots were obtained directly from the respective MCNP output files, example of which is shown in Appendix D. This portion of output file includes the number of neutron reactions in the sensor per source neutron and their uncertainties in the corresponding time bins. The total number of reactions across all time bins is also listed, which corresponds to the total area of the curve. For example, in the moderated $\mathrm{BF}_{3}$ detector, $1 \mathrm{MeV}$ neutrons have $1.51896 \mathrm{E}-05$ reactions per incident neutron in total (with a fractional uncertainty of 0.0042 ). The incident surface area of the detector multiplied by this number gives the response of detector. The response of a detector is defined as counts per unit fluence $\left[\mathrm{cm}^{2}\right]$. The calculated response function of the moderated $\mathrm{BF}_{3}$ detectors for incident from the side is given in Fig. 4. le. It shows that the detector is most sensitive to $1 \mathrm{MeV}$ neutrons. The statistical uncertainties in the calculations of all the neutron arrival time distributions for moderated $\mathrm{BF}_{3}$ detector are 


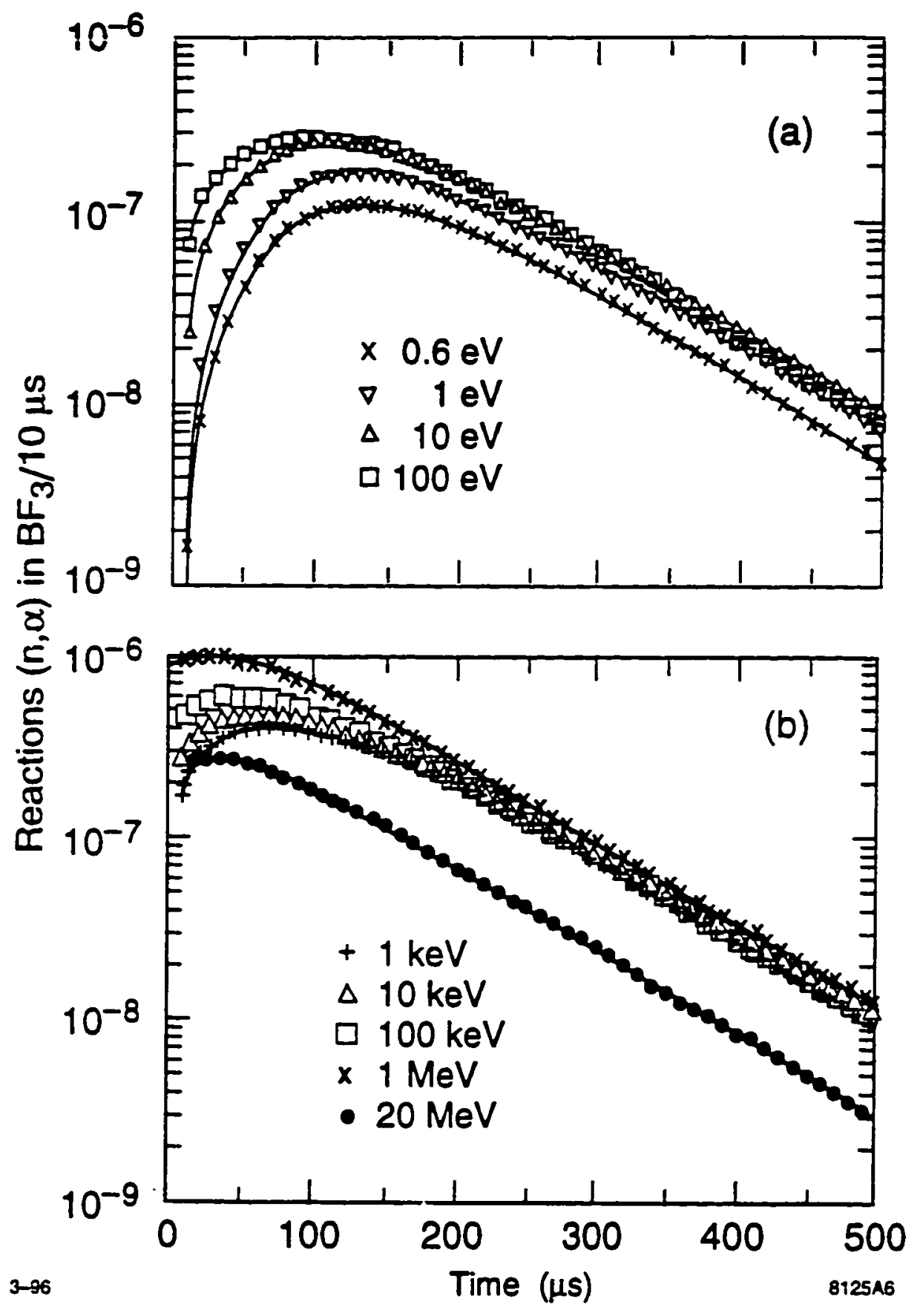

Figs. 4.1 a and $b$ MCNP-calculated neutron arrival time distribution in the moderated $\mathrm{BF}_{3}$ detector for monoenergetic neutrons. (a) for $0.6 \mathrm{eV}-100 \mathrm{eV}$ (b) for $1 \mathrm{keV}-20 \mathrm{MeV}$. 


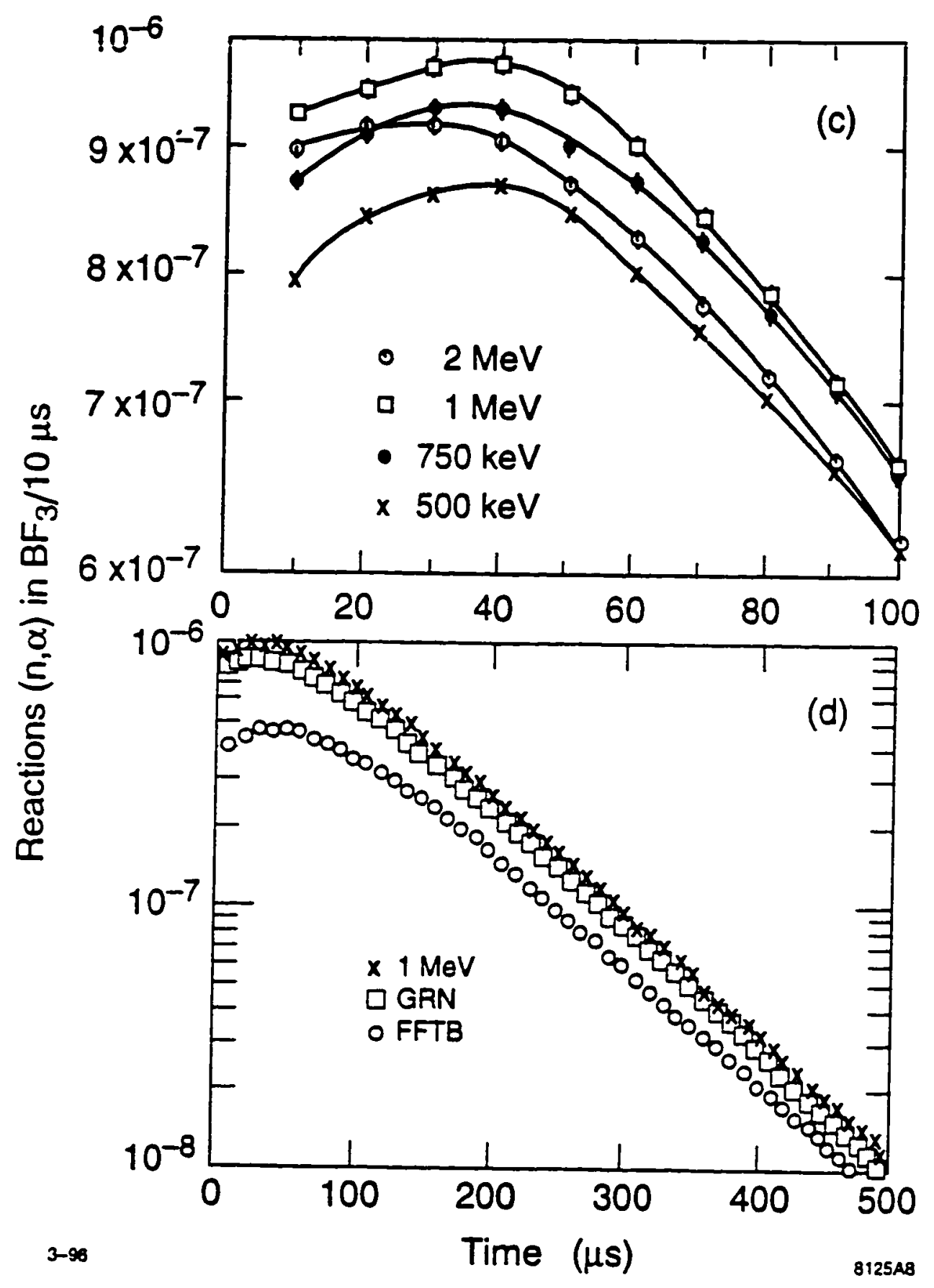

Figs. 4.1 $\mathrm{c}$ and d. MCNP-calculated neutron arrival time distribution in the moderated $\mathrm{BF}_{3}$ detector (c) $500 \mathrm{keV}-2 \mathrm{MeV}$ (d) for GRN and FFTB measured neutron spectrum. 


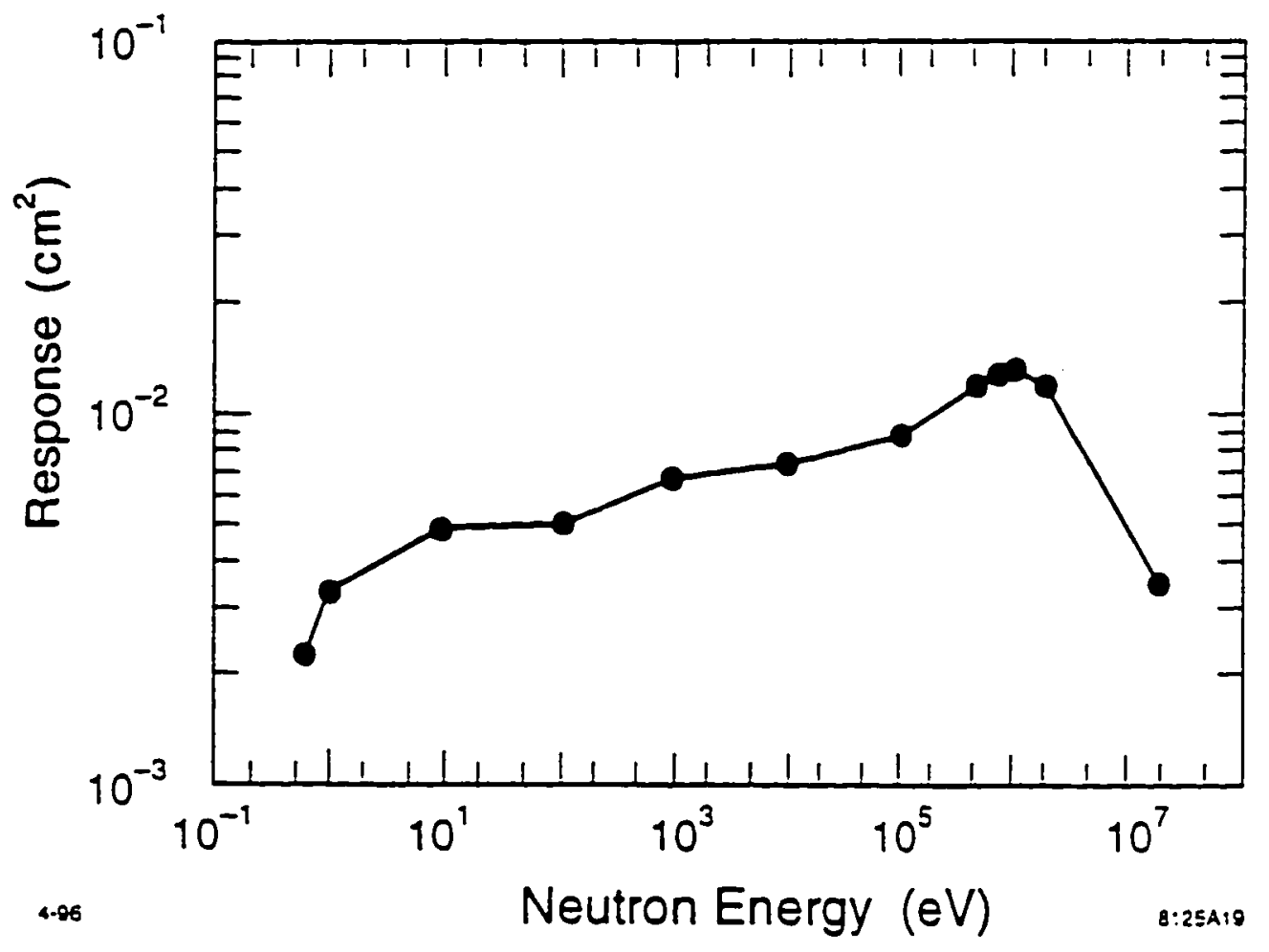

Fig. 4.1e. $\mathrm{MCNP}$-calculated response of the moderated $\mathrm{BF}_{3}$ detector for the neutrons incident from the side. 
less than $4 \%$ for most cases. Note that there is a noticeable peak shift in various neutron arrival time distributions for different neutron energies. It is clear from Figs. $4.1 \mathrm{a}, \mathrm{b}$ and $\mathrm{c}$ that the high energy neutrons are reaching the sensor faster than the lower energy neutrons. For example, $1 \mathrm{eV}$ neutrons have a peak at approximately $140 \mu \mathrm{s}$ (Fig. $4.1 \mathrm{a}$ ), whereas $1 \mathrm{MeV}$ neutrons have a peak at about $40 \mu \mathrm{s}$ (Fig. $4.1 \mathrm{c}$ ). The shift in the peak may be explained qualitatively as follows.

When high energy neutrons (e.g., $1 \mathrm{MeV}$ ) enter the detector, they penetrate deep into the polyethylene moderator as nearly uncollided fast neutrons, because of the low interaction cross section (larger mean free path). Therefore, the slowing down interactions of the fast neutrons may occur relatively near the sensor. The resulting slow neutrons have to diffuse through a short distance to reach the sensor and be detected.

On the other hand, the low energy neutron (e.g. $1 \mathrm{eV}$ ) do not travel deep into polyethylene before their first interaction because of their smaller mean free path. They lose most of the initial kinetic energy and become thermalized near the surface. These slowed-down neutrons have to diffuse through a thicker moderator layer to reach the sensor, compared to the case of incident fast neutrons. Therefore, low energy incident neutrons will be detected later in time than the high energy neutrons.

The total area in Fig.4.1d shows that the moderated $\mathrm{BF}_{3}$ detector has higher response to $1 \mathrm{MeV}$ neutrons than the GRN or FFTB spectra. This is due to the fact that GRN and FFTB spectra include a number of neutrons above and below $1 \mathrm{MeV}$ neutrons respectively, to which the detector is less sensitive. The neutron arrival time distributions 
for GRN and measured FFTB spectra are very close to that of $1 \mathrm{MeV}$ neutrons, and both spectra have peak at $1 \mathrm{MeV}$. Figure 4. le shows that the MCNP-calculated response of moderated $\mathrm{BF}_{3}$ detector has a peak at $\mathrm{l} \mathrm{MeV}$. The response calculations are in close agreement with those done in the past for a similar moderated $\mathrm{BF}_{3}$ detector having a $6 \mathrm{~cm}$ radial thickness of polyethylene moderator (Kosako 1985).

\subsubsection{Anderson-Braun remmeter results}

Figures $4.2 \mathrm{a}$ and $\mathrm{b}$ show the results of MCNP simulation of monoenergetic neutrons incident on the $\mathrm{AB}$ remmeter. Fig. $4.2 \mathrm{c}$ shows a comparison of results of the GRN and FFTB spectrum with that of $1 \mathrm{MeV}$ monoenergetic neutrons. The data for these plots were obtained in a similar way as that used for the moderated $\mathrm{BF}_{3}$ detector. Calculated response of the $A B$ remmeter is shown in Fig. 4.2d, which has a peak at about $2 \mathrm{MeV}$. The data used to generate response curves of the $A B$ remmeter, as well as that of moderated $\mathrm{BF}_{\mathbf{z}}$ detector are given in Appendix $\mathrm{E}$. The statistical uncertainties in the $\mathrm{AB}$ remmeter calculations are very small $(<3 \%)$, except for the $10 \mathrm{eV}$ neutrons for which the error is about $18 \%$.

The shift in the peak of the arrival time distribution as a function of neutron energy is also observed here. The remmeter also has a lower response for the FFTB spectrum than for GRN and 1-MeV neutrons. 

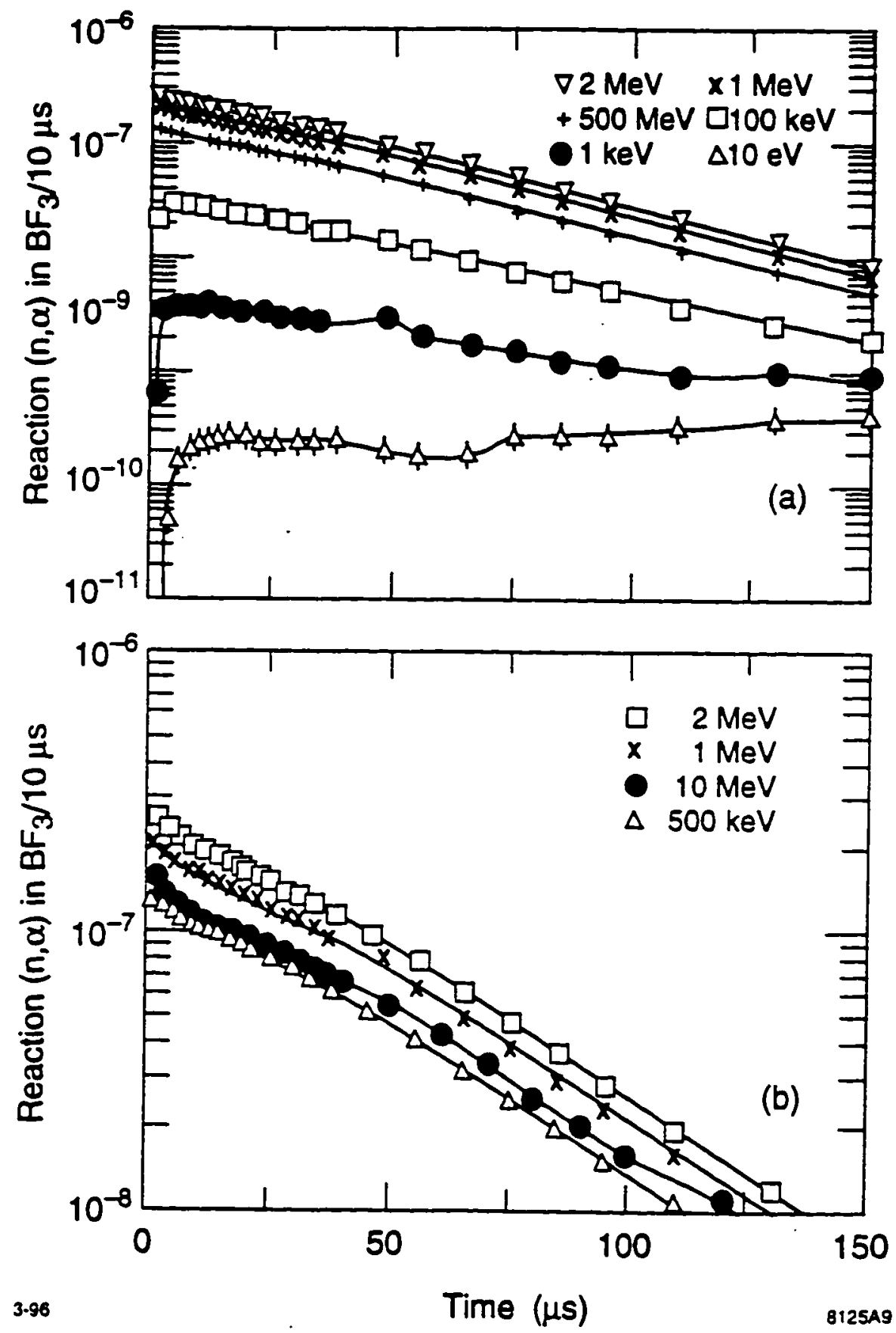

Figs. 4.2a and $b$. MCNP-calculated neutron arrival time distribution in the AndersonBraun remmeter for monoenergetic neutrons.(a) for $10 \mathrm{eV}-2 \mathrm{MeV}$ (b) for $500 \mathrm{keV}-10$ $\mathrm{MeV}$. 


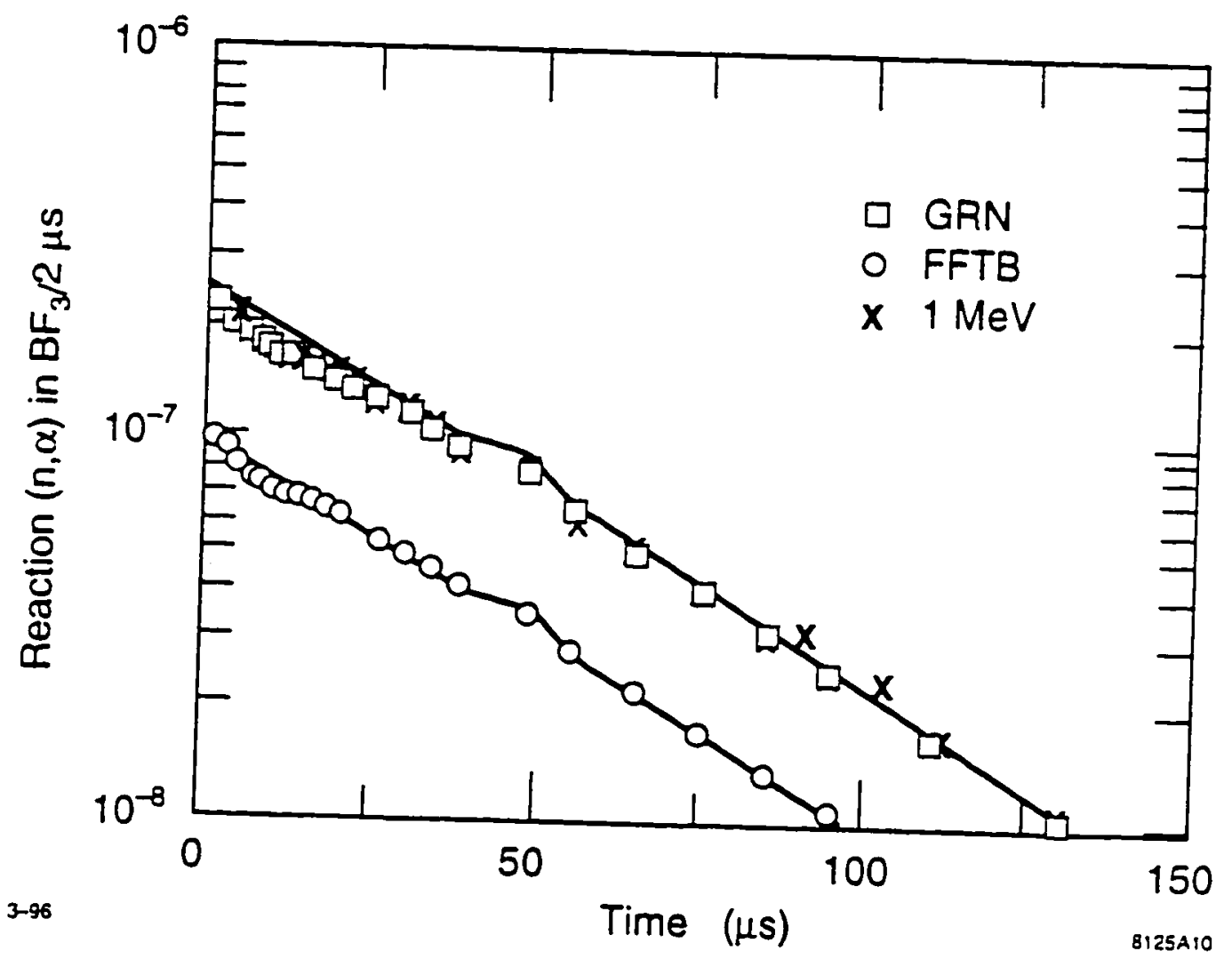

Fig. 4.2c. MCNP-calculated neutron arrival time distribution in the Anderson-Braun remmeter for GRN and FFTB measure neutron spectrum compared with that for $1 \mathrm{MeV}$ neutrons. 


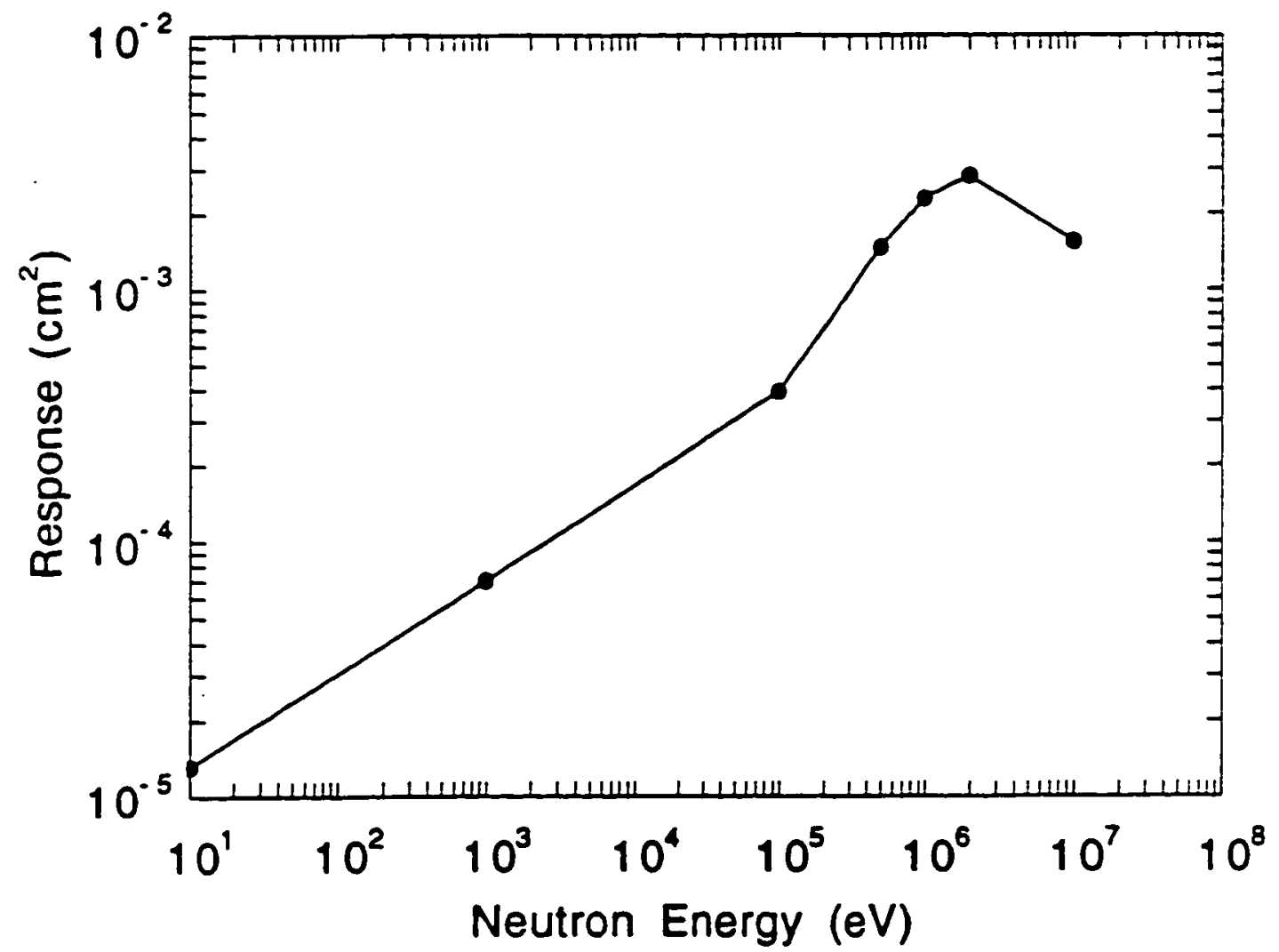

Fig. 4.2d. MCNP-calculated response of the Anderson-Braun remmeter. Note that the counts are per unit fluence. 


\subsubsection{Bonner Sphere results}

The results of the MCNP calculations for some monoenergetic, GRN and FFTB neutrons are given in Figs. 4.3 through 4.8 for the Bonner Sphere detectors with polyethylene spheres of $2^{\prime \prime}, 3^{\prime \prime}, 5^{\prime \prime}, 8^{\prime \prime}, 10^{\prime \prime}$, and $12^{\prime \prime}$ diameter, respectively.

The neutron arrival time distributions for all the Bonner Spheres have a spike at the beginning of the curve. It might be explained by the fact that the cross section for ${ }^{6} \mathrm{Li}$ $(\mathrm{n}, \alpha)^{3} \mathrm{H}$ reaction has a pronounced resonance peak at a neutron energy of about $250 \mathrm{keV}$. Therefore, the incident fast neutrons component around the resonant region entering the LiI crystal is detected with a higher efficiency. However, the $1 \mathrm{keV}$ curve also exhibit a spike, so the above explanation does not fully account for this effect. It should be noted that neutron arrival time distribution curves of 8", 10", and 12" Bonner Sphere for GRNs have been smoothed.

The responses of these detectors are tabulated in Tables 1, 2, and 3. The calculated response functions for all spheres are reasonable and have the expected shape. The smaller Bonner Spheres (2", 3", and $5^{\prime \prime}$ ) have high response at low energies (few eV to $\mathrm{keV}$ ) whereas the response peak shifts to higher energies ( $100 \mathrm{keV}$ to few $\mathrm{MeV}$ ) for larger spheres ( $8^{\prime \prime}, 10^{\prime \prime}$, and $\left.12^{\prime \prime}\right)$.

For the Bonner Sphere detector with a larger moderator, the arrival of low energy neutrons is delayed by a significant amount of time. For example $0.1 \mathrm{eV}$ neutrons in $10^{\prime \prime}$ Bonner Sphere detector arrive at the sensor after $140 \mu$ s (Fig. 4.7a) and for 12" Bonner Sphere, even $100 \mathrm{eV}$ neutrons do not reach the detector until $140 \mu \mathrm{s}$ (Fig. 4.8a). 


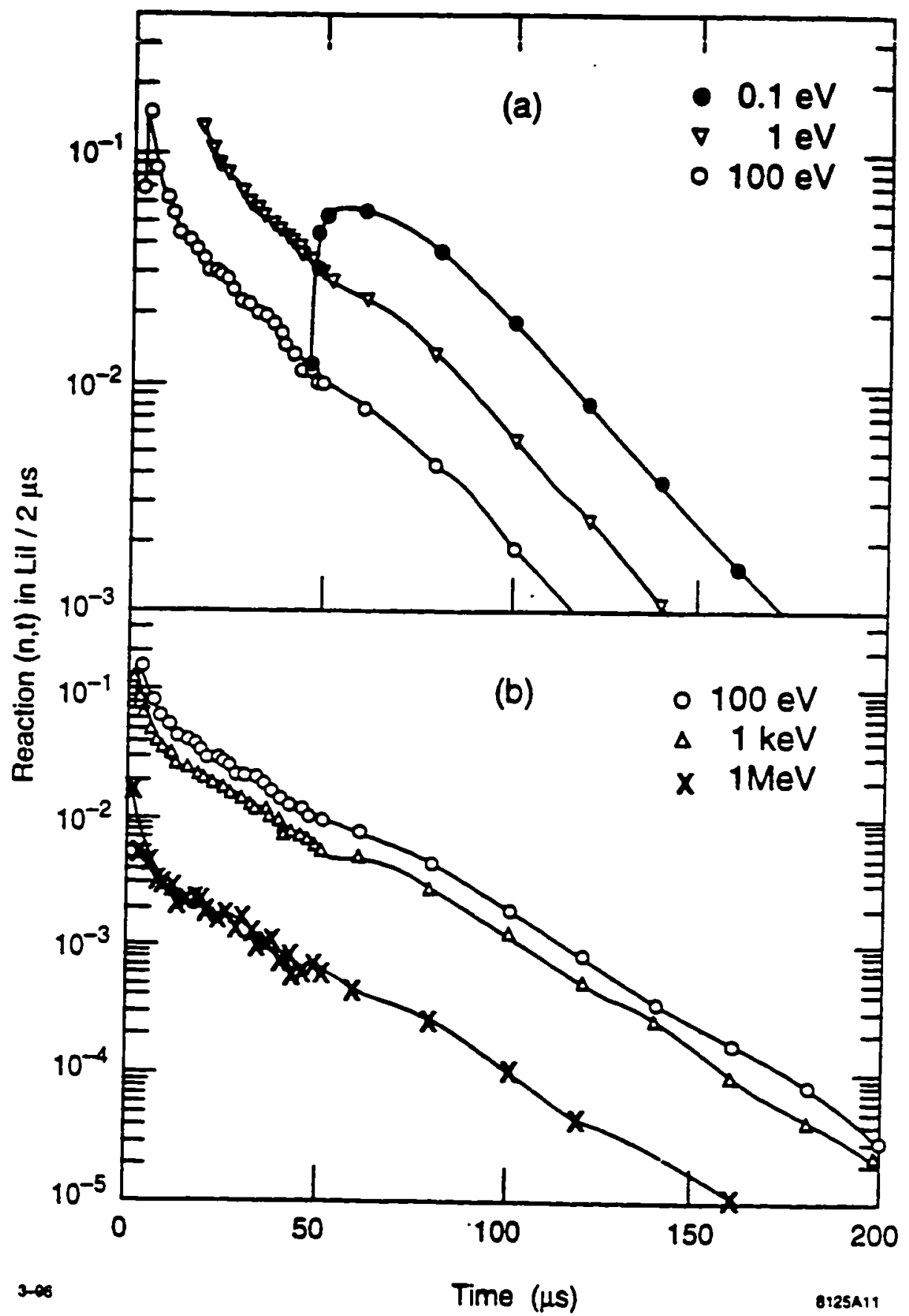

Figs. 4.3a and $b$. MCNP-calculated neutron arrival time distribution in the 2" Bonner Sphere detector for monoenergetic neutrons.

(a) for $0.1 \mathrm{eV}-100 \mathrm{eV}$ (b) for $100 \mathrm{eV}-1 \mathrm{MeV}$. 


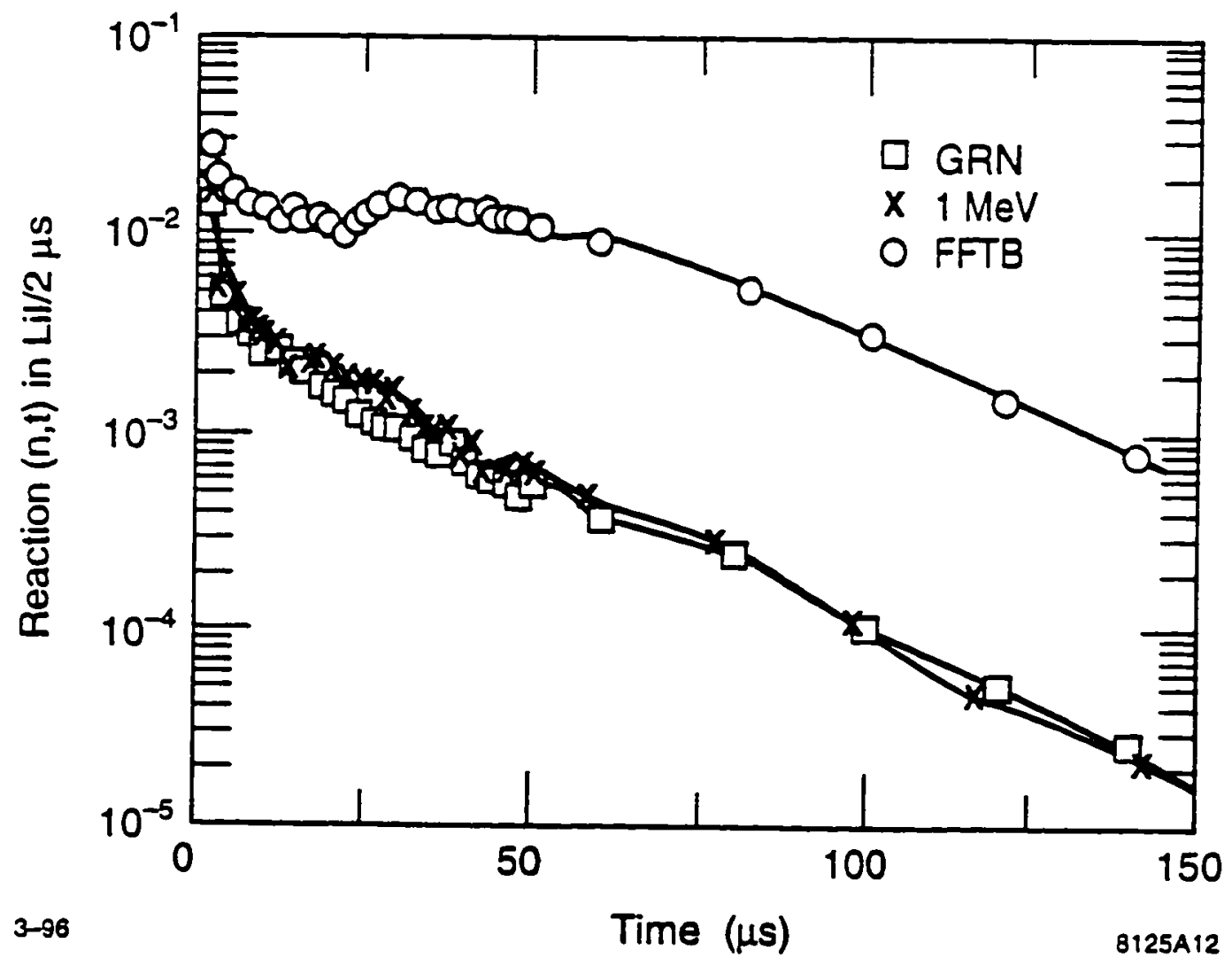

Fig. 4.3c. MCNP-calculated neutron arrival time distribution in the 2 " Bonner Sphere detector for GRN and FFTB measured neutron spectrum compared with that for $1 \mathrm{MeV}$ neutrons. 


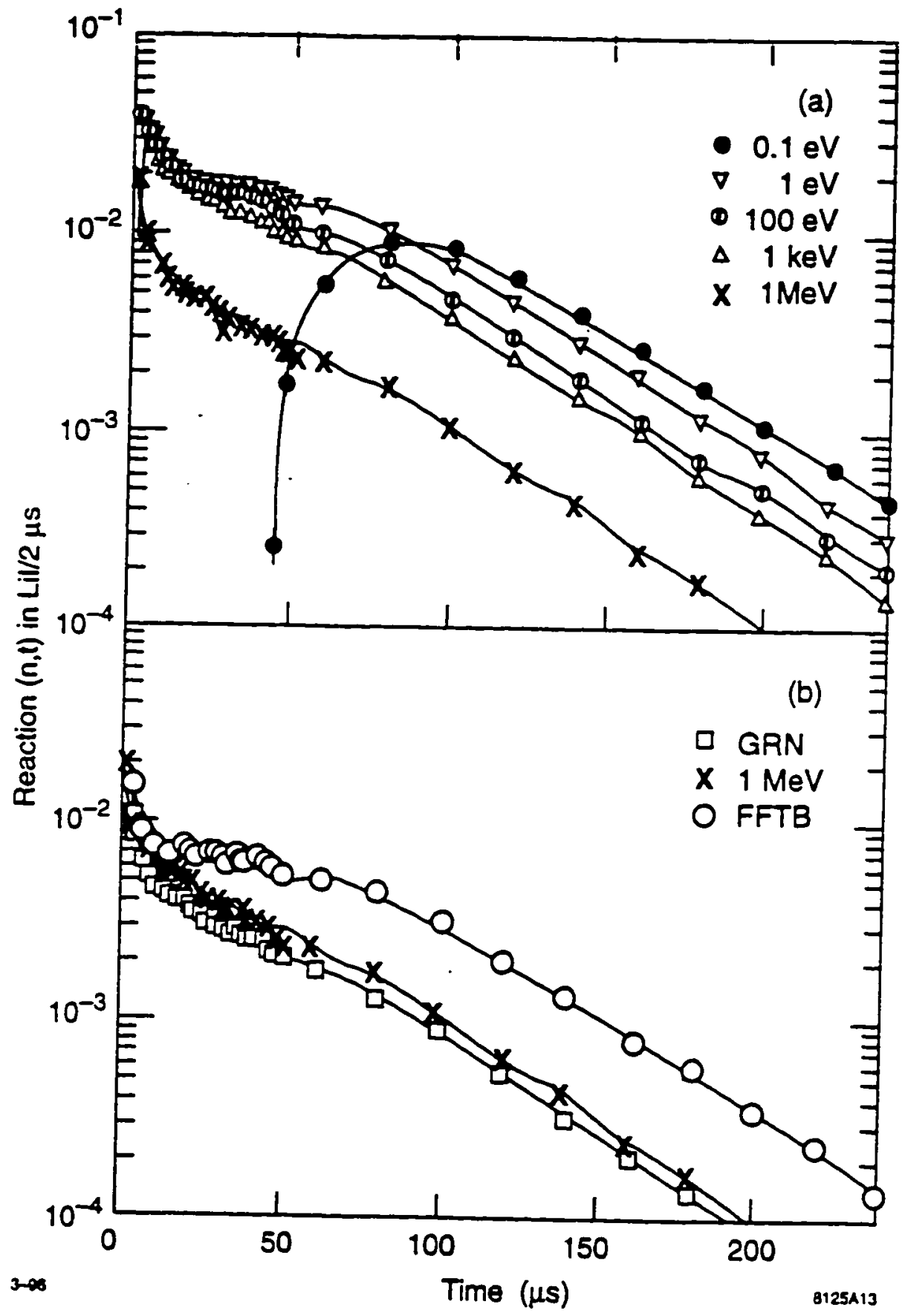

Figs. 4.4a and $b$. MCNP-calculated neutron arrival time distribution in the $3^{\prime \prime}$ Bonner Sphere detector (a) for monoenergetic neutrons (b) for GRN and FFTB measured neutron spectrum compared with that for I MeV neutrons. 


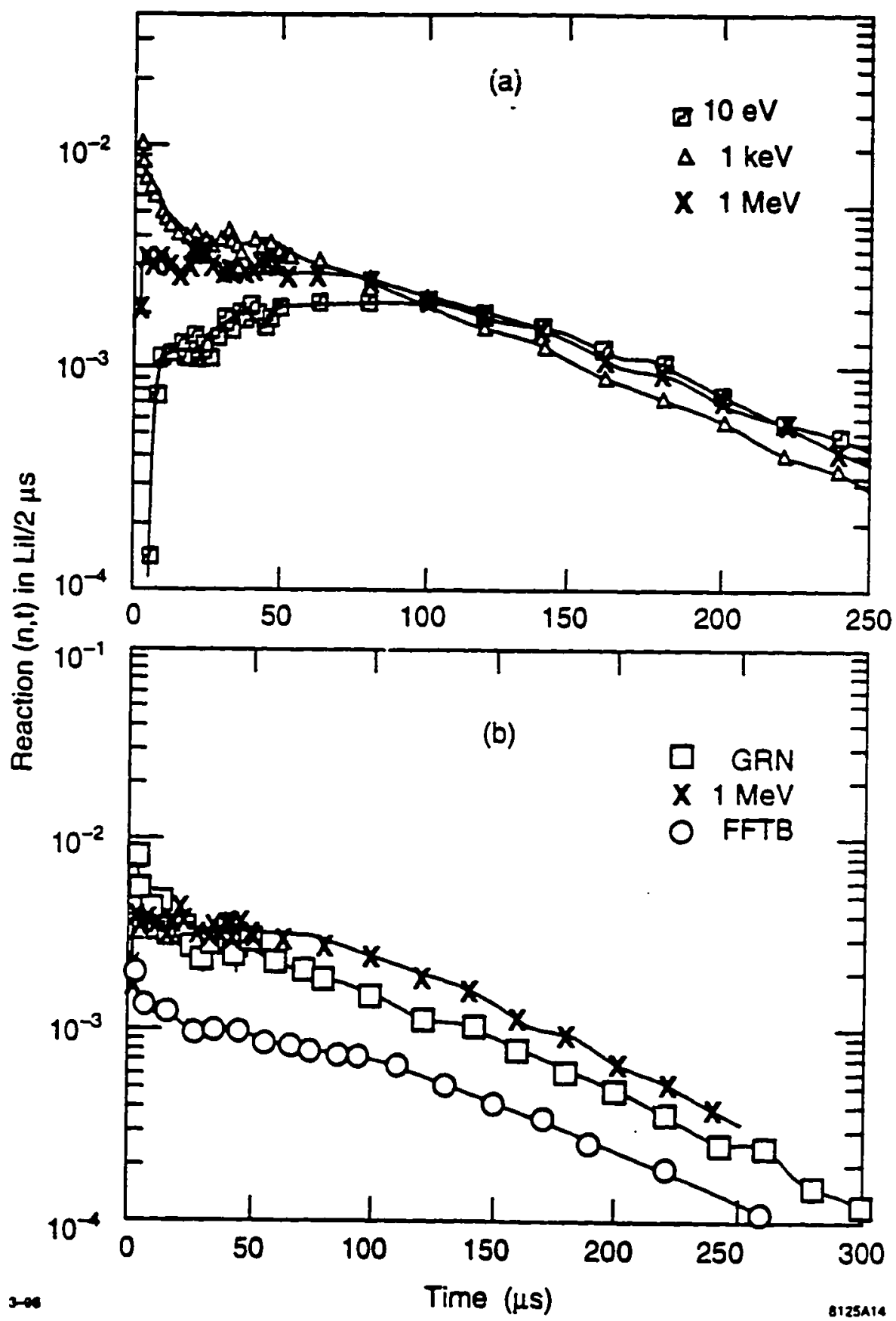

Figs. 4.5a and b MCNP-calculated neutron arrival time distribution in the $5^{\prime \prime}$ Bonner Sphere detector (a) for monoenergetic neutrons (b) for GRN and FFTB measured neutron spectrum compared with that for $1 \mathrm{MeV}$ neutrons. 


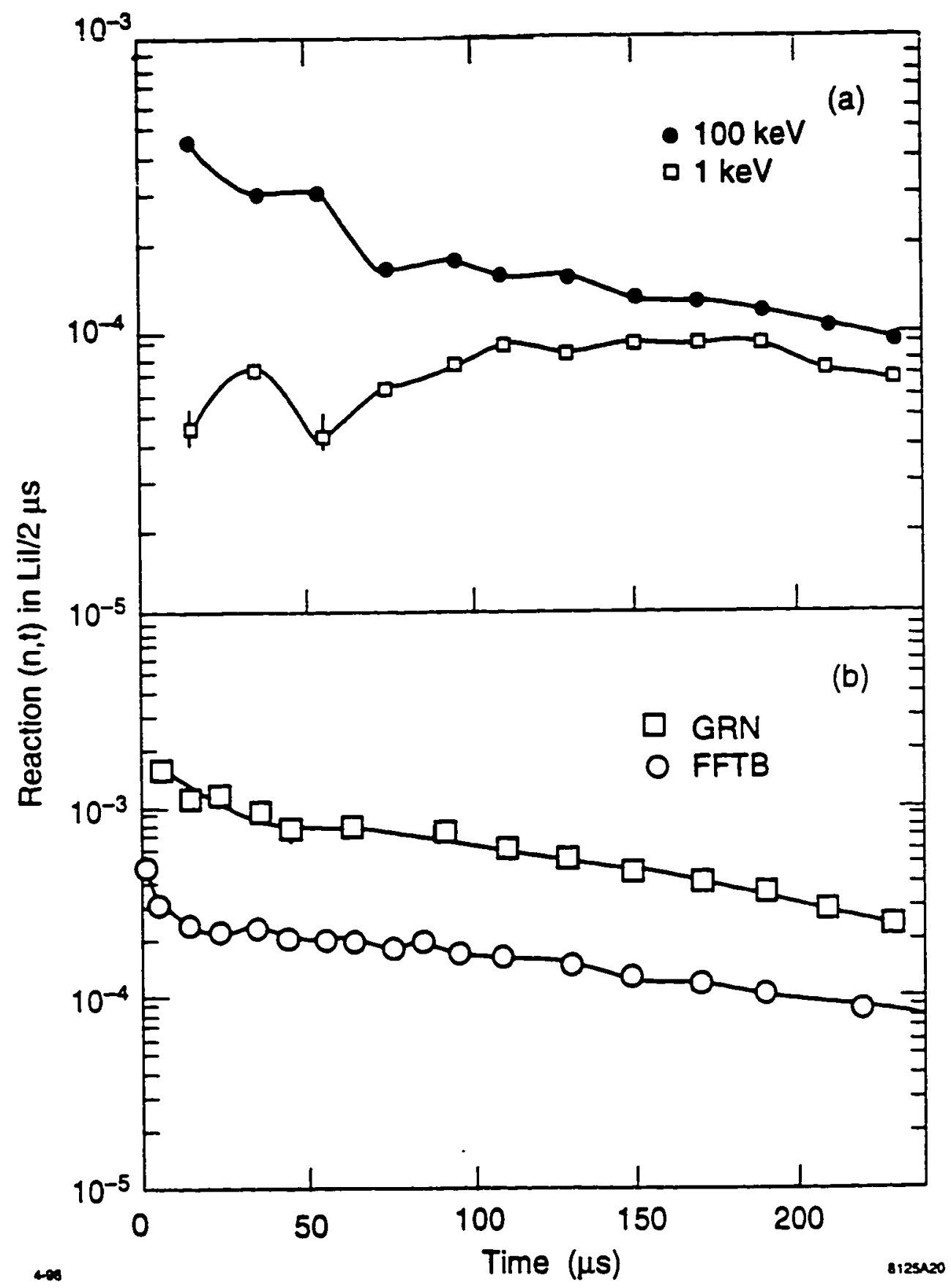

Figs. 4.6a and b MCNP-calculated neutron arrival time distribution in the $8^{\prime \prime}$ Bonner Sphere detector (a) for monoenergetic neutrons (b) for GRN and FFTB measured neutron spectrum. 


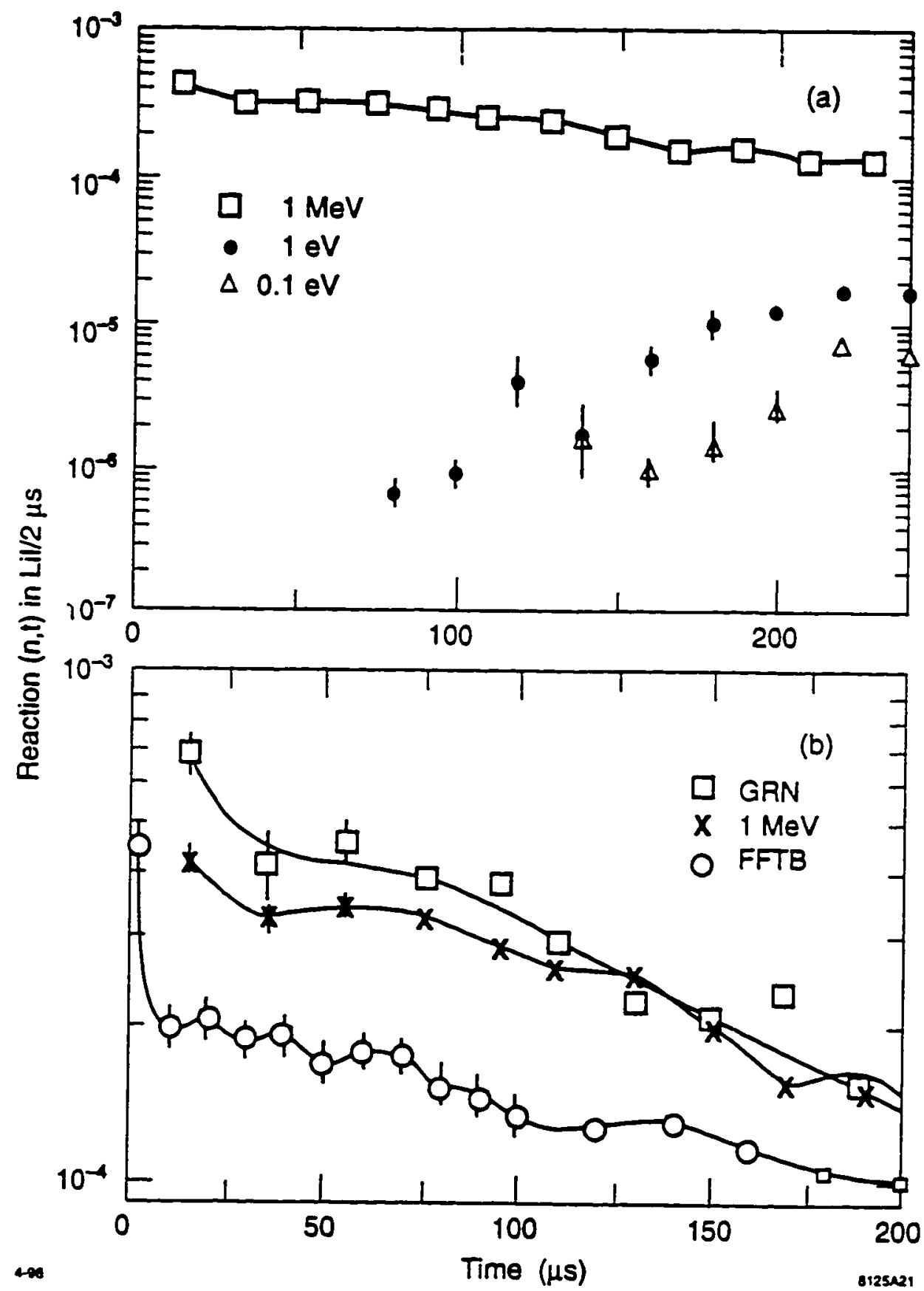

Figs. 4.7a and $b$ MCNP-calculated neutron arrival time distribution in the $10^{\prime \prime}$ Bonner Sphere detector (a) for monoenergetic neutrons.(b) for GRN and FFTB measured neutron spectrum compared with that for $\mathrm{l} \mathrm{MeV}$ neutrons. 


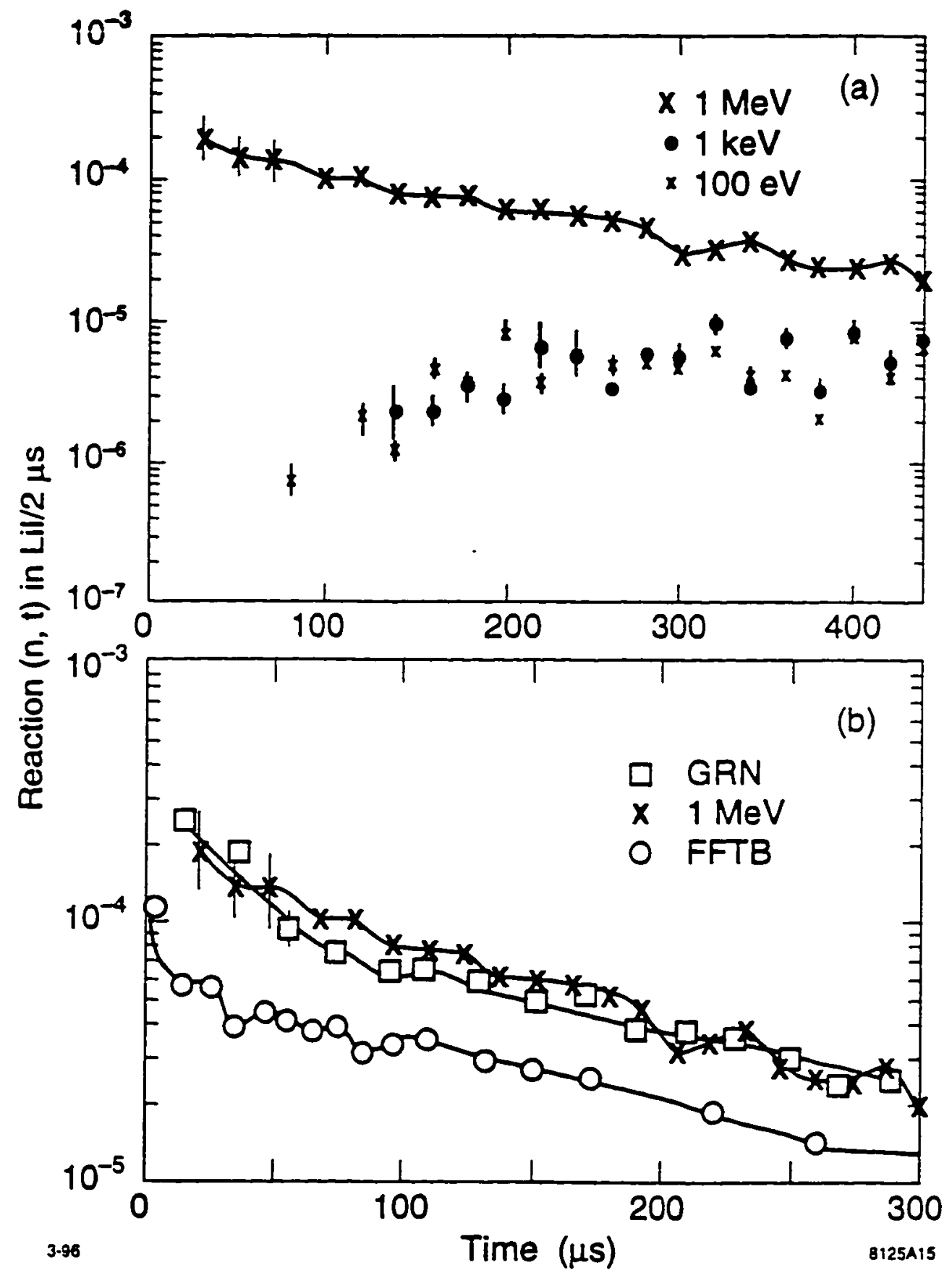

Figs. 4.8a and $b$ MCNP-calculated neutron arrival time distribution in the 12 " Bonner Sphere detector (a) for monoenergetic neutrons (b) for GRN and FFTB measured neutron spectrum compared with that for $1 \mathrm{MeV}$ neutrons. 
Table 1. MCNP Calculations of response of $2^{\prime \prime}$ and 3 "Bonner Sphere delector.

\begin{tabular}{|c|c|c|c|c|c|c|}
\hline \multirow[b]{2}{*}{ Energy } & \multicolumn{3}{|c|}{ 2" Bonner Sphere $\left(20.30 \mathrm{~cm}^{2}\right)^{\mathrm{a}}$} & \multicolumn{3}{|c|}{$3^{\prime \prime}$ Bonner Sphere $\left(45.60 \mathrm{~cm}^{2}\right)^{\mathrm{a}}$} \\
\hline & Counts $^{b}$ & $\begin{array}{c}\text { Error } \\
(\%)\end{array}$ & $\begin{array}{c}\text { Response }^{c} \\
{\left[\mathrm{~cm}^{2}\right]}\end{array}$ & Counts $^{b}$ & $\begin{array}{l}\begin{array}{l}\text { Error } \\
(\%)\end{array} \\
\end{array}$ & $\begin{array}{c}\text { Response }{ }^{c} \\
{\left[\mathrm{~cm}^{2}\right]}\end{array}$ \\
\hline $0.1 \mathrm{eV}$ & 1.09 & 0.43 & 22.03 & 0.38 & 0.66 & 17.12 \\
\hline $0.6 \mathrm{eV}$ & - & - & - & - & - & - \\
\hline $1 \mathrm{eV}$ & 1.52 & 0.35 & 30.82 & 0.66 & 0.51 & 30.15 \\
\hline $10 \mathrm{eV}$ & - & - & - & - & - & - \\
\hline $100 \mathrm{eV}$ & 1.00 & 0.56 & 20.34 & 0.72 & 0.49 & 32.72 \\
\hline I keV & 0.68 & 0.50 & 13.84 & 0.61 & 0.53 & 27.90 \\
\hline $10 \mathrm{keV}$ & - & - & - & - & - & - \\
\hline $100 \mathrm{keV}$ & - & 一 & - & 一 & - & - \\
\hline $500 \mathrm{keV}$ & - & - & - & - & - & - \\
\hline $750 \mathrm{keV}$ & - & - & 一 & - & 一 & - \\
\hline $1 \mathrm{MeV}$ & 0.07 & 1.54 & 1.38 & 0.19 & 0.96 & 8.47 \\
\hline $2 \mathrm{MeV}$ & - & - & - & - & - & - \\
\hline $10 \mathrm{MeV}$ & - & - & - & - & - & - \\
\hline $20 \mathrm{MeV}$ & - & - & - & - & - & - \\
\hline GRN & 0.06 & 1.62 & 1.15 & 0.15 & 0.62 & 6.65 \\
\hline FFTB & 0.49 & 0.22 & 10.00 & 0.33 & 0.71 & 15.22 \\
\hline
\end{tabular}

a

cross sectional area of the detector

b number of reactions per source neutron in $\mathrm{LiI}$

c

per unit fluence 
Table 2. MCNP Calculations of response of $5^{\prime \prime}$ and 8 "Bonner Sphere detector.

\begin{tabular}{|c|c|c|c|c|c|c|}
\hline \multirow[b]{2}{*}{ Energy } & \multicolumn{3}{|c|}{ 5" Bonner Sphere $\left(126.70 \mathrm{~cm}^{2}\right)^{a}$} & \multicolumn{3}{|c|}{$8^{n}$ Bonner Sphere $\left(324.30 \mathrm{~cm}^{2}\right)^{\mathrm{s}}$} \\
\hline & Counts $^{b}$ & $\begin{array}{c}\text { Error } \\
(\%)\end{array}$ & $\begin{array}{c}\text { Response }^{c} \\
{\left[\mathrm{~cm}^{2}\right]}\end{array}$ & Counts $^{b}$ & $\begin{array}{l}\text { Error } \\
(\%)\end{array}$ & $\begin{array}{c}\text { Response }^{c} \\
{\left[\mathrm{~cm}^{2}\right]}\end{array}$ \\
\hline $0.1 \mathrm{eV}$ & - & - & - & - & - & - \\
\hline $0.6 \mathrm{eV}$ & - & - & - & - & - & - \\
\hline $1 \mathrm{eV}$ & - & - & - & - & - & - \\
\hline $\mathrm{I} 0 \mathrm{eV}$ & 0.17 & 0.90 & 21.06 & 0.01 & 9.95 & 3.12 \\
\hline $100 \mathrm{eV}$ & - & - & - & - & - & - \\
\hline $1 \mathrm{keV}$ & 0.23 & $0 . .82$ & 29.06 & 0.02 & 7.50 & 6.50 \\
\hline $10 \mathrm{keV}$ & - & - & - & - & - & - \\
\hline $100 \mathrm{keV}$ & 一 & 一 & - & 0.04 & 5.80 & 13.21 \\
\hline $500 \mathrm{keV}$ & - & - & - & 0.07 & 1.10 & 21.90 \\
\hline $750 \mathrm{keV}$ & - & 一 & - & - & - & - \\
\hline $\mathrm{I} \mathrm{MeV}$ & 0.21 & 0.84 & 26.52 & 0.08 & 2.96 & 27.17 \\
\hline $2 \mathrm{MeV}$ & - & - & - & 0.09 & 0.73 & 29.31 \\
\hline $10 \mathrm{MeV}$ & - & - & - & - & - & - \\
\hline $20 \mathrm{MeV}$ & - & - & - & - & - & - \\
\hline GRN & 0.19 & 1.35 & 24.07 & 0.08 & 2.13 & 26.05 \\
\hline FFTB & 0.16 & 0.49 & 19.79 & 0.04 & 0.87 & 14.20 \\
\hline
\end{tabular}

a

cross sectional area of the detector

b

number of reactions per source neutron in Lil

${ }^{c}$ per unit fluence 
Table 3. MCNP Calculations of response of $10^{\prime \prime}$ and $12^{\prime \prime}$ Bonner Sphere detector.

\begin{tabular}{|c|c|c|c|c|c|c|}
\hline \multirow[b]{2}{*}{ Energy } & \multicolumn{3}{|c|}{ IU" Bonner Sphere $\left(506.7 \mathrm{~cm}^{2}\right)^{2}$} & \multicolumn{3}{|c|}{ 12" Bonner Sphere $\left(729.7 \mathrm{~cm}^{3}\right)^{i}$} \\
\hline & Counts & $\begin{array}{c}\text { Error } \\
(\%)\end{array}$ & $\begin{array}{c}\text { Response }^{\mathrm{C}} \\
{\left[\mathrm{cm}^{2}\right]}\end{array}$ & Counts ${ }^{b}$ & $\begin{array}{l}\text { Error } \\
(\%)\end{array}$ & $\begin{array}{c}\text { Response }{ }^{c} \\
{\left[\mathrm{~cm}^{2}\right]}\end{array}$ \\
\hline $0.1 \mathrm{eV}$ & $4.23 E-04$ & 15.10 & 0.21 & - & - & - \\
\hline $0.6 \mathrm{eV}$ & - & - & - & - & - & - \\
\hline $\mathrm{leV}$ & $1.23 E-03$ & 8.60 & 0.61 & - & - & - \\
\hline $10 \mathrm{eV}$ & - & - & - & - & - & - \\
\hline $100 \mathrm{eV}$ & - & - & - & $8.70 \mathrm{E}-04$ & 9.60 & 0.63 \\
\hline $1 \mathrm{keV}$ & $2.90 \mathrm{E}-03$ & 5.90 & 1.47 & $1.02 \mathrm{E}-03$ & 8.20 & 0.74 \\
\hline $10 \mathrm{keV}$ & - & - & - & - & - & - \\
\hline $100 \mathrm{keV}$ & - & - & - & - & - & - \\
\hline $500 \mathrm{keV}$ & $2.01 \mathrm{E}-02$ & 1.70 & 10.16 & $7.93 \mathrm{E}-03$ & 4.90 & 5.78 \\
\hline $750 \mathrm{keV}$ & - & - & - & - & - & - \\
\hline $1 \mathrm{MeV}$ & $3.20 E-02$ & 2.06 & 16.21 & $1.4+E-02$ & 2.90 & 10.51 \\
\hline $2 \mathrm{MeV}$ & 4.4IE-02 & 1.20 & 1.20 & $2.44 \mathrm{E}-02$ & 3.60 & 17.83 \\
\hline $10 \mathrm{MeV}$ & - & - & - & - & - & - \\
\hline $20 \mathrm{MeV}$ & - & - & - & - & - & - \\
\hline GRN & $4.50 E-02$ & 4.20 & 22.80 & $2.12 E-02$ & 5.10 & 15.47 \\
\hline FFTB & $1.96 \mathrm{E}-02$ & 1.61 & 9.91 & $9.76 \mathrm{E}-03$ & 1.85 & 7.12 \\
\hline
\end{tabular}

cross sectional area of the detector

b number of reactions per source neutron in LiI

c per unit fluence 


\subsection{Measurements and comparisons}

The measurements for the arrival time distributions were made for the moderated $\mathrm{BF}_{3}$ detector, $\mathrm{AB}$ remmeter, $2^{\prime \prime}$ and $10^{\prime \prime}$ Bonner Sphere detectors. The results are shown in Figs. $4.9-4.12$, respectively. The integral plots were used for this analysis. The measurement results are compared with the corresponding integral plots of the calculated neutron arrival time distributions (i.e. MCNP calculated fractional cumulative counts for measured FFTB spectrum).

Figure. $4.9 \mathrm{a}$ and $\mathrm{b}$ shows the comparison between the measured and calculated results for the moderated $\mathrm{BF}_{3}$ detector. In this figure, calculation results include the FFTB measured neutron spectrum and the MCNP-calculated GRN spectra with three different values of parameter ' $\mathrm{a}$ ' $(\mathrm{a}=1 \mathrm{MeV}, 1.2 \mathrm{MeV}, 0.8 \mathrm{MeV})$. The MCNP calculation results for different spectra are very similar. The measurements (for the FFTB measured neutron spectrum) differ from calculations by $\approx 20 \%$ at $100 \mu \mathrm{s}$. The error in both calculation and measurements are less than $2 \%$.

Figure $4.10 \mathrm{a}$ and $\mathrm{b}$ shows the comparison between measurement and calculation results for an $A B$ remmeter. There is a difference of $\approx 15 \%$ at $100 \mu \mathrm{s}$. The statistical error in both calculations as well as measurements are less than $1.5 \%$. The discrepancies in the moderated $\mathrm{BF}_{3}$ detector and $\mathrm{AB}$ remmeter comparison are real and unexplained at present, which need further investigation. 

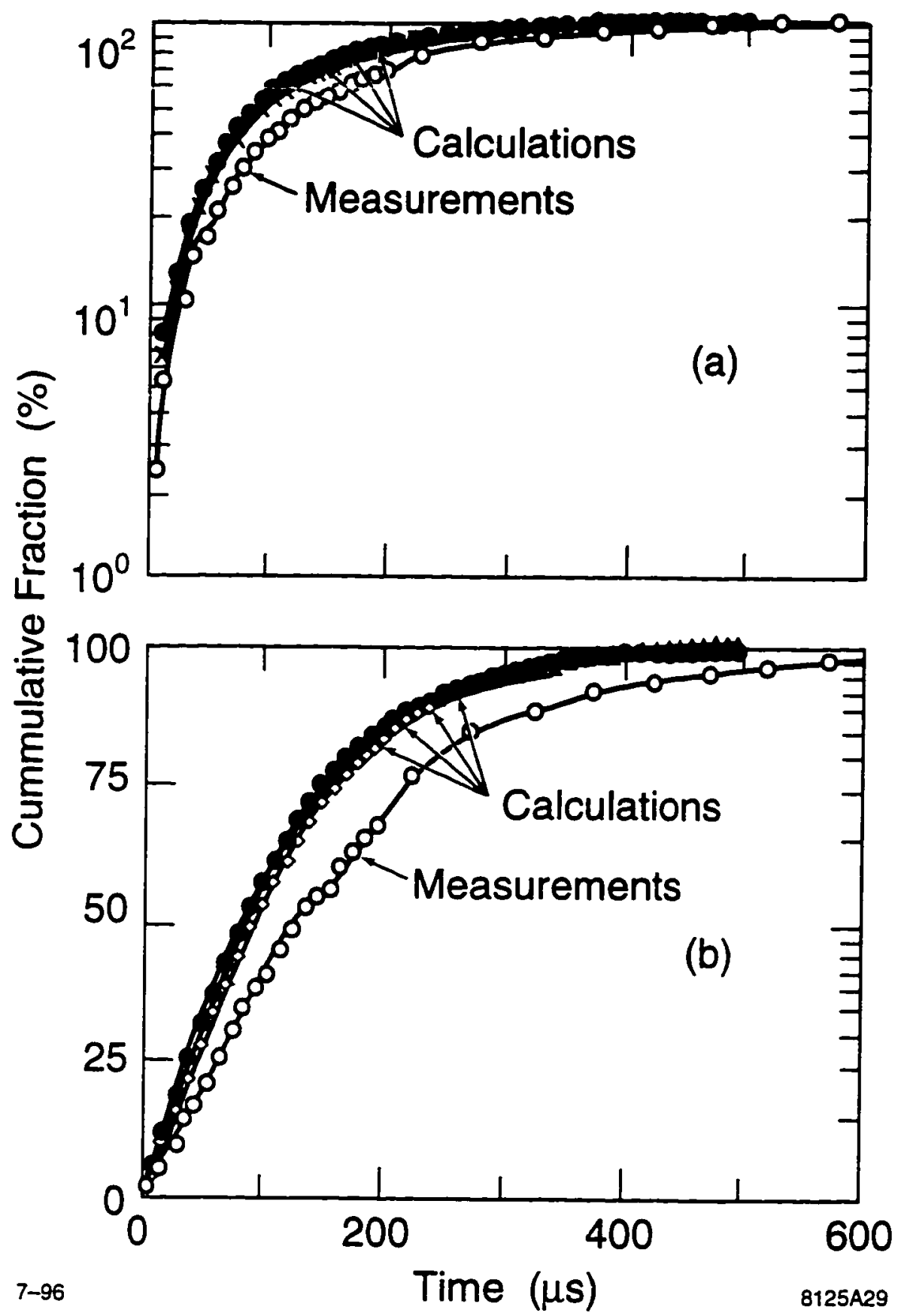

Figs. 4.9a and $b$ A comparison between the MCNP-calculations and the measurement of fractional cumulative counts versus the neutron arrival time for the FFTB spectrum incident on the moderated $\mathrm{BF}_{3}$ detector (a) a semilog plot (b) a linear plot. The calculations include the results for three GRN spectra (for $a=1 \mathrm{MeV}, \mathrm{a}=1.2 \mathrm{MeV}$, and $\mathrm{a}$ $=0.8 \mathrm{MeV}$ ) and the measured FFTB neutron spectrum. 


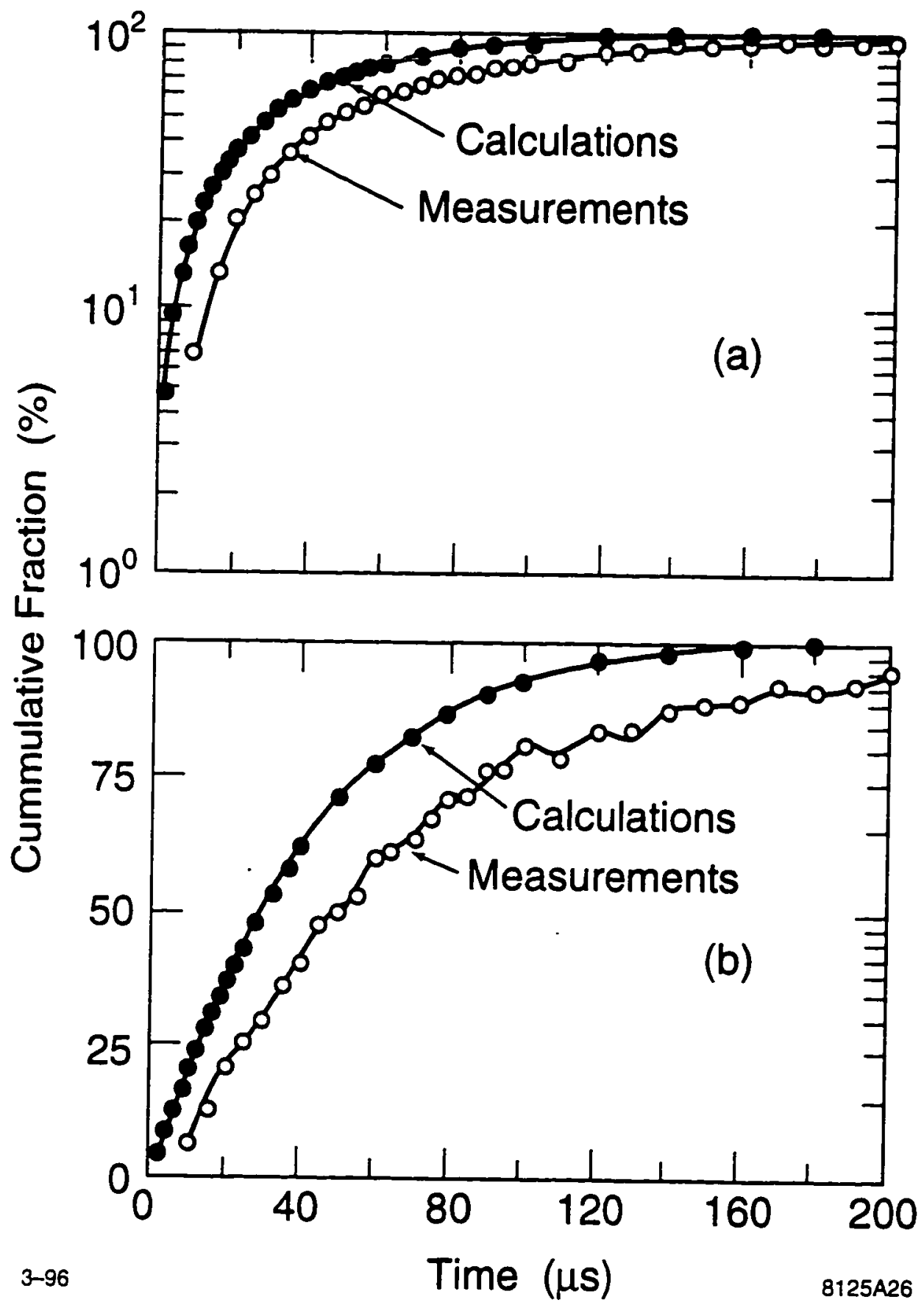

Figs. 4.10a and $b$ A comparison between the MCNP-calculations and the measurement of fractional cumulative counts versus the neutron arrival time for the FFTB spectrum incident on the Anderson-Braun remmeter (a) a semilog plot (b) a linear plot. 


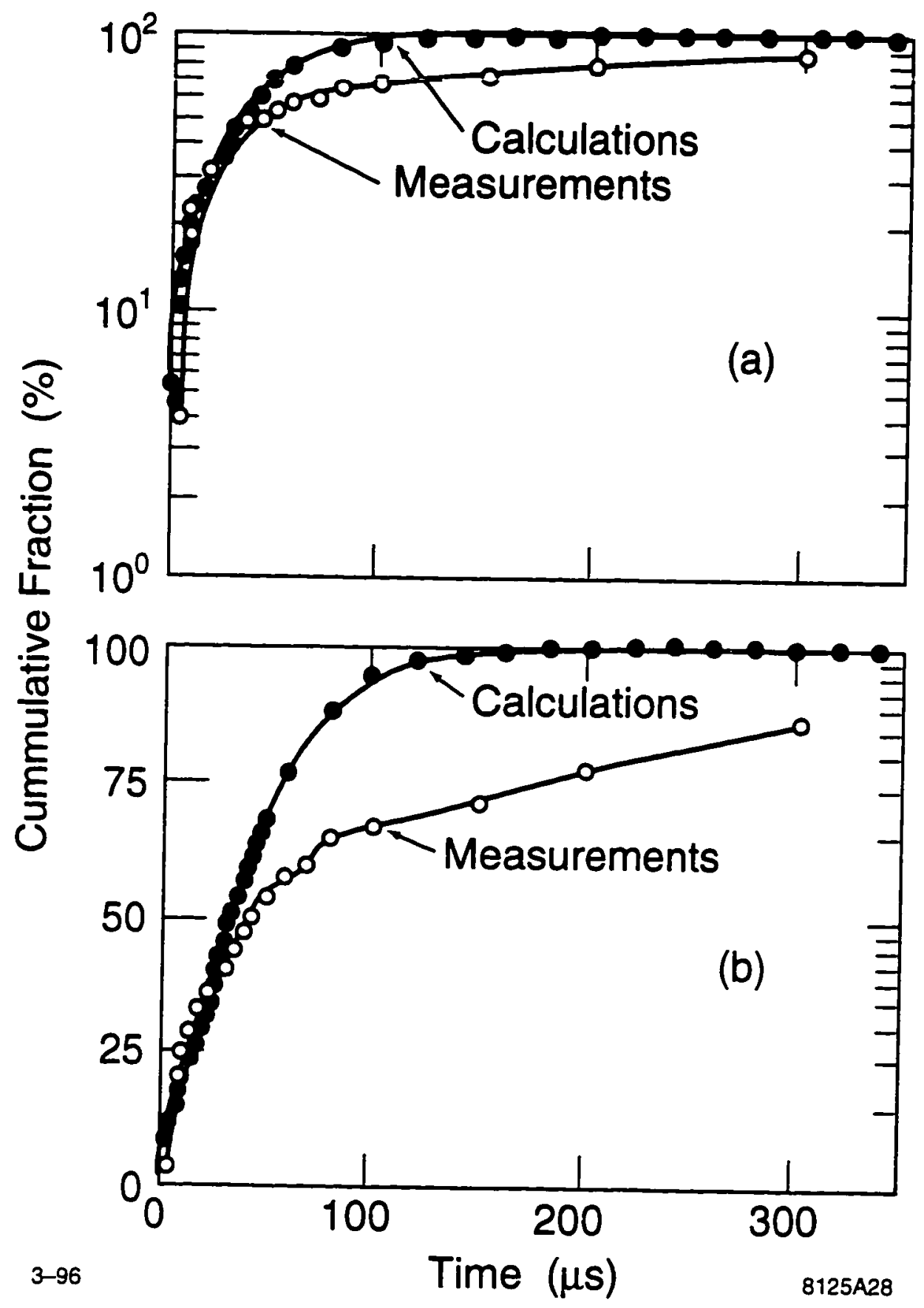

Figs. 4.11a and $b$ A comparison between the MCNP-calculations and the measurement of fractional cumulative counts versus the neutron arrival time for the FFTB spectrum incident on the 2" Bonner Sphere detector (a) a semilog plot (b) a linear plot. 


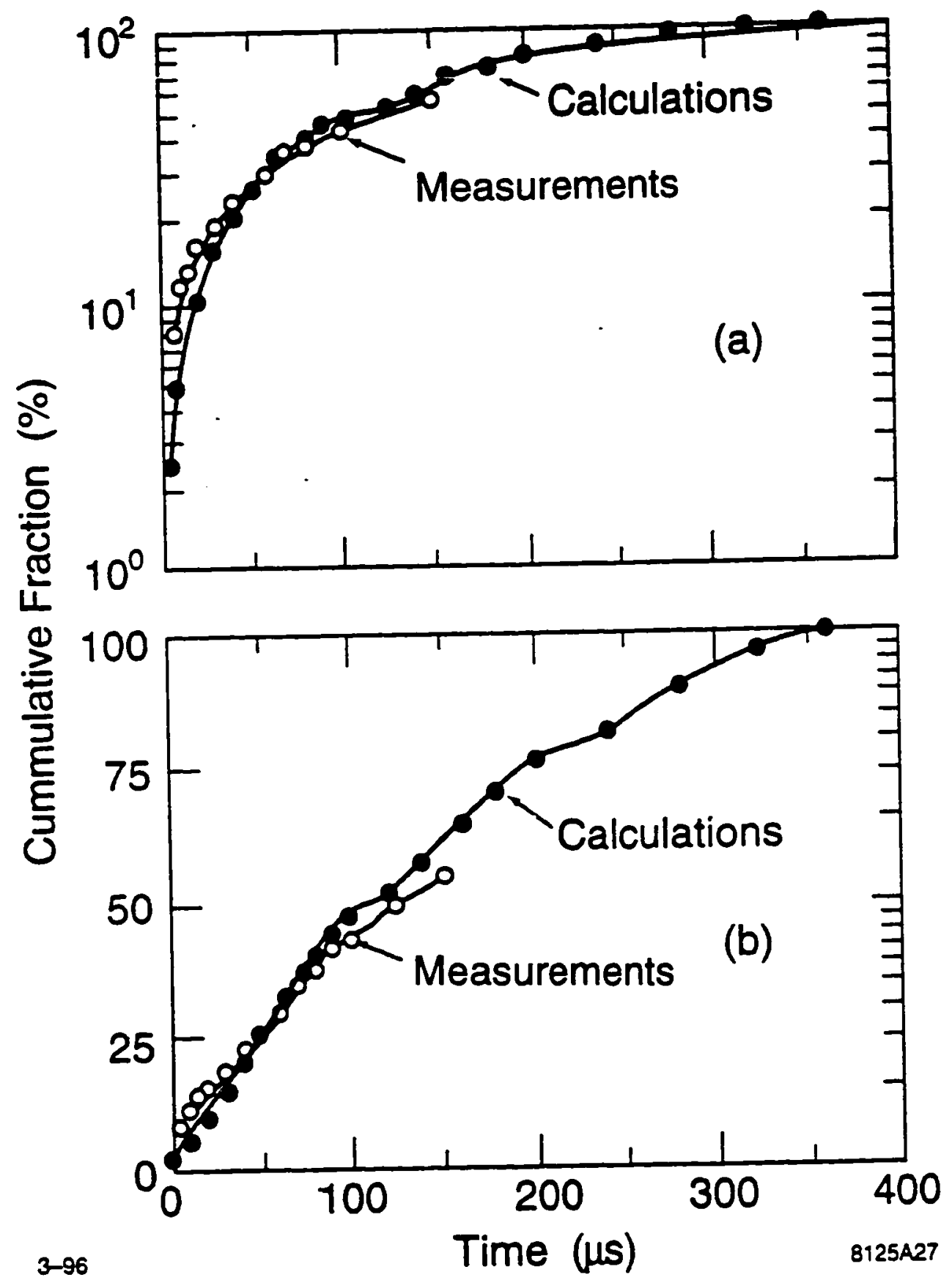

Figs. 4.12a and $b$ A comparison between the MCNP-calculations and the measurement of fractional cumulative counts versus the neutron arrival time for the FFTB spectrum incident on the $10^{\prime \prime}$ Bonner Sphere detector (a) a semilog plot (b) a linear plot. 
Figure 4. $1 \mathrm{la}$ and $\mathrm{b}$ is a comparison between the calculations and measurements for $2 "$ Bonner Sphere detector. It is clear that the 2" Bonner Sphere calculations reach to $100 \%$ much faster than the measurement result. This can be explained by the fact that the 2" Bonner Sphere is most sensitive to low energy neutrons and, in the real case, low energy neutrons in FFTB spectrum are delayed by the timing effect of concrete shielding before they reach the detector. In the calculations, this shielding effect is not accounted for. If this delay of slow neutrons is simulated in MCNP calculation, the results would agree better with the measurements.

A comparison in the calculations and measurements results for the $10^{\prime \prime}$ Bonner Sphere detector is shown in Fig. 4.12a and b. The calculation results are in close agreement with the measurement.

\subsection{Calculation of $\mathrm{T}$-values}

The mean arrival time (T-value) is the inverse of the slope of the neutron arrival time distributions. T-values for various detectors have been calculated for FFTB spectrum from the calculated neutron arrival time distributions and are shown in the Table 4.

Table 4. Mean neutron arrival time for FFTB spectrum

Detector

$\mathrm{BF}_{3}$ detector

$A B$ remmeter

Bonner Spheres

$2^{\prime \prime}$

3 "

$5 "$

8 "

$10^{\prime \prime}$

12 "
T-value $(\mu s)$ 


\section{SUMMARY AND CONCLUSIONS}

\subsection{Summary}

Neutron arrival time distributions for three moderating type fast neutron detectors were calculated using the MCNP4A code. The detectors were a moderated $\mathrm{BF}_{3}$ detector, an Anderson-Braun remmeter and Bonner Sphere detectors (of 2", 3", 5", 8", 10", and 12" diameter). Each of the detectors had polyethylene as a moderator.

The input files including modeled geometry of each detector and various source descriptions were developed. Number of reactions in the sensor per source neutron as a function of neutron arrival time was calculated. Output from the MCNP code was represented as the neutron arrival time distributions. The response of each detector was also calculated. Results obtained were discussed for each detector.

The results from the calculations were compared with the measurement made at FFTB (46.7 GeV e' beam dump at SLAC) for the moderated $\mathrm{BF}_{3}$ detector, $\mathrm{AB}$ remmeter and Bonner Spheres (of 2" and 10" diameter).

The calculated and measured results for large Bonner Sphere (10") are in close agreement. The disagreement for 2" Bonner Sphere is due the delay in low-energy neutrons before they reach the detector. The delay is introduced by the timing effect of the concrete shielding, which was not accounted for in the MCNP calculations.

The difference in the measured and calculated results for the moderated $\mathrm{BF}_{3}$ detector and $\mathrm{AB}$ remmeter can not be completely explained at present. 


\subsection{Conclusions}

From the study of the MCNP calculated neutron arrival time distributions of moderating type detectors, the following conclusions can be drawn:

1) The neutron arrival time distributions exhibit the exponential behavior

2) For each detector in this study there is a noticeable shift in the peak of the neutron arrival time distributions, depending on the thickness of the moderator and the energy of the incident neutrons. The delay in the arrival of low-energy incident neutrons at the sensor is due to low-energy neutrons becoming thermalized near the surface of the detector. The thermal neutrons in this case have to diffuse through a larger moderator thickness to reach the sensor as compared to the case of fast incident neutrons

3) The neutron arrival time distributions are spectrum dependent.

4) The neutron arrival time distributions for Bonner Spheres (LiI sensor) detectors show a spike at the beginning of the curve. It might be partially explained by the presence of pronounced response peak around $250 \mathrm{keV}$ in the cross section of ${ }^{6} \mathrm{Li}(\mathrm{n}, \alpha)^{3} \mathrm{H}$ reaction.

5) The MCNP calculated responses of all detectors are reasonable and have the shapes as expected. The moderated $\mathrm{BF}_{3}$ detector has a peak at $\mathrm{IMeV}$. $\mathrm{AB}$ remmeter has a peak at about $2 \mathrm{MeV}$. The Bonner Sphere detectors of small diameters (2", 3", and 5") have high response to low energy incident neutron and larger spheres ( $8^{\prime \prime}, 10^{\prime \prime}$, and $\left.12^{\prime \prime}\right)$ have high response to high energy incident neutrons.

6) Except for the 2" and 3" Bonner Sphere all the detectors show lower response to the FFTB measured spectrum than GRNs and $1 \mathrm{MeV}$ monoenergetic neutrons. 
The 10" Bonner Sphere detector shows a good agreement between the measured and calculated results. The comparison of 2" Bonner Sphere calculations and the measurements results shows discrepancies. The calculations show that neutrons reach the detector faster than that of the measurement results.

Similar calculations were done by Liu (1996) for an experimental setup at ANL (Argonne National Laboratory). He simulated the ANL room geometry with MCNP for 2" Bonner Sphere detector. The timing effect of the concrete shielding was also incorporated in the calculations. The arrival time distribution results closely agree with the experimental data for that set-up.

Therefore, the discrepancies of 2" Bonner Sphere detector for our work may be explained by the fact that the low-energy neutrons are significantly deiayed through the concrete shielding in FFTB dump before entering the detector. This effect was not accounted for in our calculations and therefore, the calculated 2" sphere results do not agree with the measurements.

The comparison between the measured and calculated results for $\mathrm{AB}$ remmeter and moderated $\mathrm{BF}_{3}$ detector show discrepancies and can not be completely explained at present. However, it can be postulated that it may be due to the anisotropic response of these detectors. The measurements include neutrons incident from all sides, whereas in the calculations, neutron sources are simulated as incident only in the direction perpendicular to the detector axis. The neutrons incident parallel to the detector axis could have longer arrival time and can reach the sensor later in time as found in the measurements. Further studies are needed for the confirmation of this postulate. 


\subsection{Further studies}

The first further work would be the calculation of the timing effect of the concrete shielding and its effect on the arrival of neutrons in various detectors. Another study would be to calculate the slopes (and therefore, T-values) of the neutron arrival time distributions for various detectors for other spectra. This could be helpful in the calculations of the dead time correction factor for moderating type fast neutron detectors operating in pulse mode in a pulsed radiation field. 


\section{REFERENCES}

Ash, W.; DeStaebler, H.; Harris, J.; and Murray, J. PEP radiation shielding tests in SLAC A Beam. SLAC-TN-77-5, PEP-252, Stanford Linear Accelerator Center, Stanford, CA; 1977.

International Commission on Radiological Protection. Data for protection against ionizing radiation for external sources. Oxford Pergamon Press, ICRP Publication 21; 1973.

International Atomic Energy Agency. Radiation safety aspects of the operation of electron linear accelerators. Technical report series no. 188, IAEA Vienna; 1979.

Jenkins, T.M. Neutron and photon measurements through concrete from a $15 \mathrm{GeV}$ electron beam on a target - comparison with model and calculations. Nucl. Instrum. Methods 159, pp. 265-288 (1979).

Knoll, G.F. Radiation detection and measurement. 2nd edition, John Wiley \& Son, Inc.; 1989.

Kosako, T.; Nakamura, T.; Iwai, S. Estimation of response function of moderating type neutron detectors by the time-of-flight method combined with a large lead pile. Nucl. Instrum. Methods in Phys. Res., A235, pp. 103-122; 1985.

Liu, J. C.; Jenkins, T. M; McCall, R. C.; Ipe, N. E. Neutron dosimetry at SLAC: neutron sources and instrumentation. Report No. SLAC-TN-91-3 Stanford Linear Accelerator Center, Stanford, CA; 1991.

Liu, J.C. Private communications. SLAC; 1996.

MCNP - A General purpose Monte Carlo N-Particle Transport Code, Version 4A, Los Alamos National Laboratory, New Mexico; 1993.

Patterson, H. W; Thomas, R. H. Accelerator health physics. Academic press, Inc. New York, NY (1973).

Rokni, S. Private communications. SLAC; 1996.

Thai, X. Private communication. SLAC; 1996. 


\section{Appendix Al}

$\mathrm{MCNP}$ input file of the moderated $\mathrm{BF}_{3}$ detector for monoenergetic neutrons $(20 \mathrm{MeV})$.

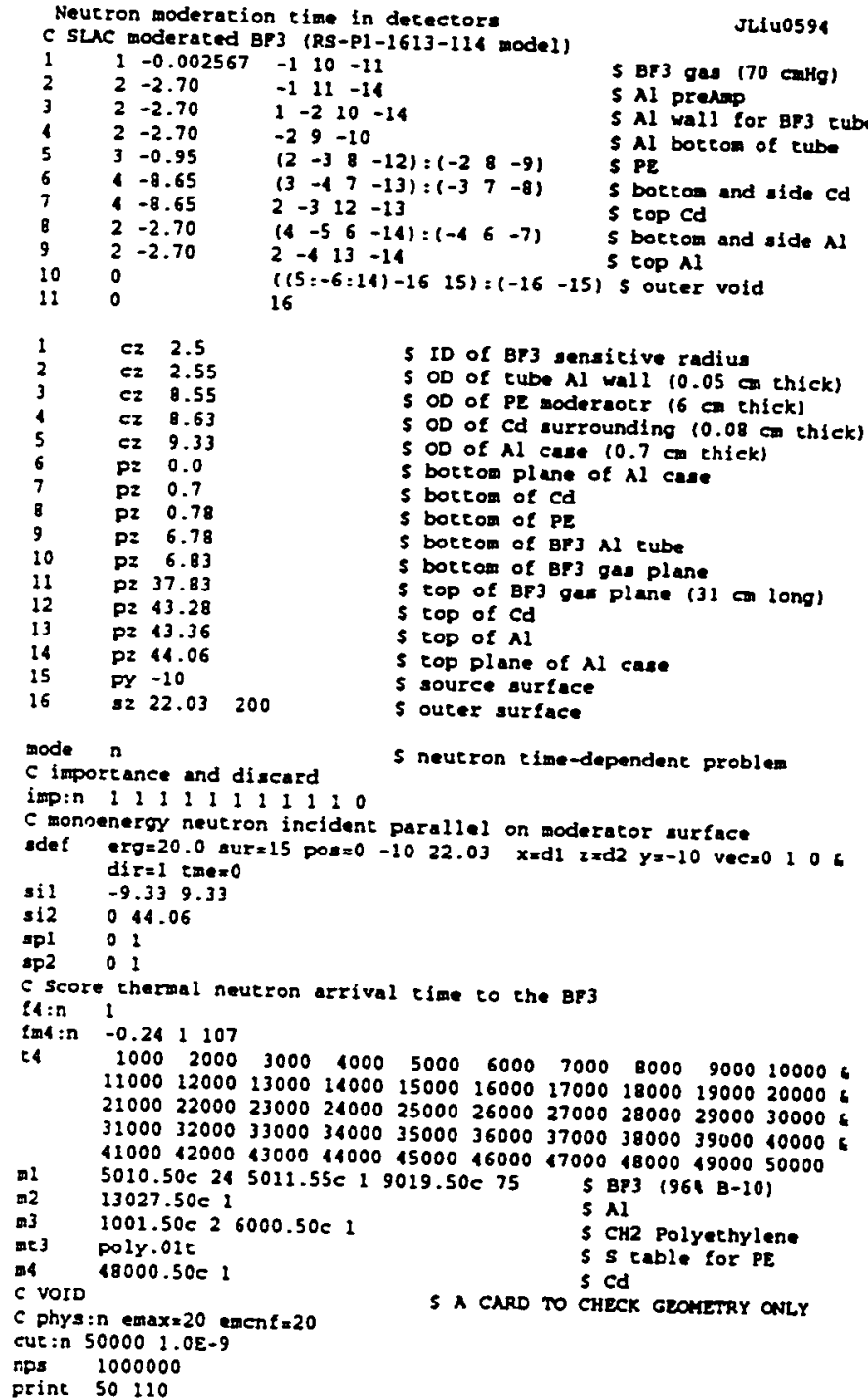




\section{Appendix A2}

MCNP input file of the moderated $\mathrm{BF}_{3}$ detector for GRNs.

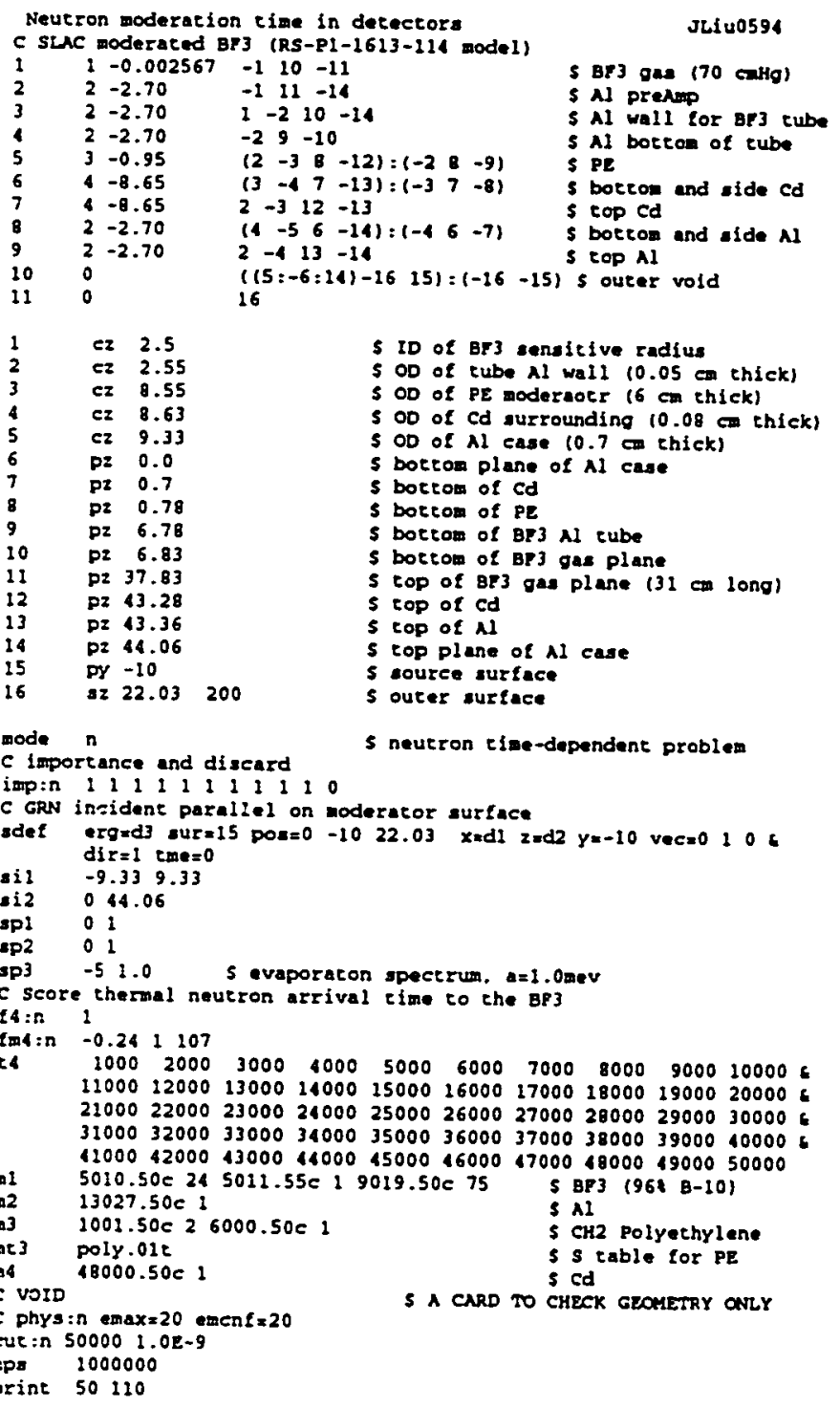




\section{Appendix A3}

MCNP input file of the moderated $\mathrm{BF}_{3}$ detector for FFTB measured neutron spectrum.

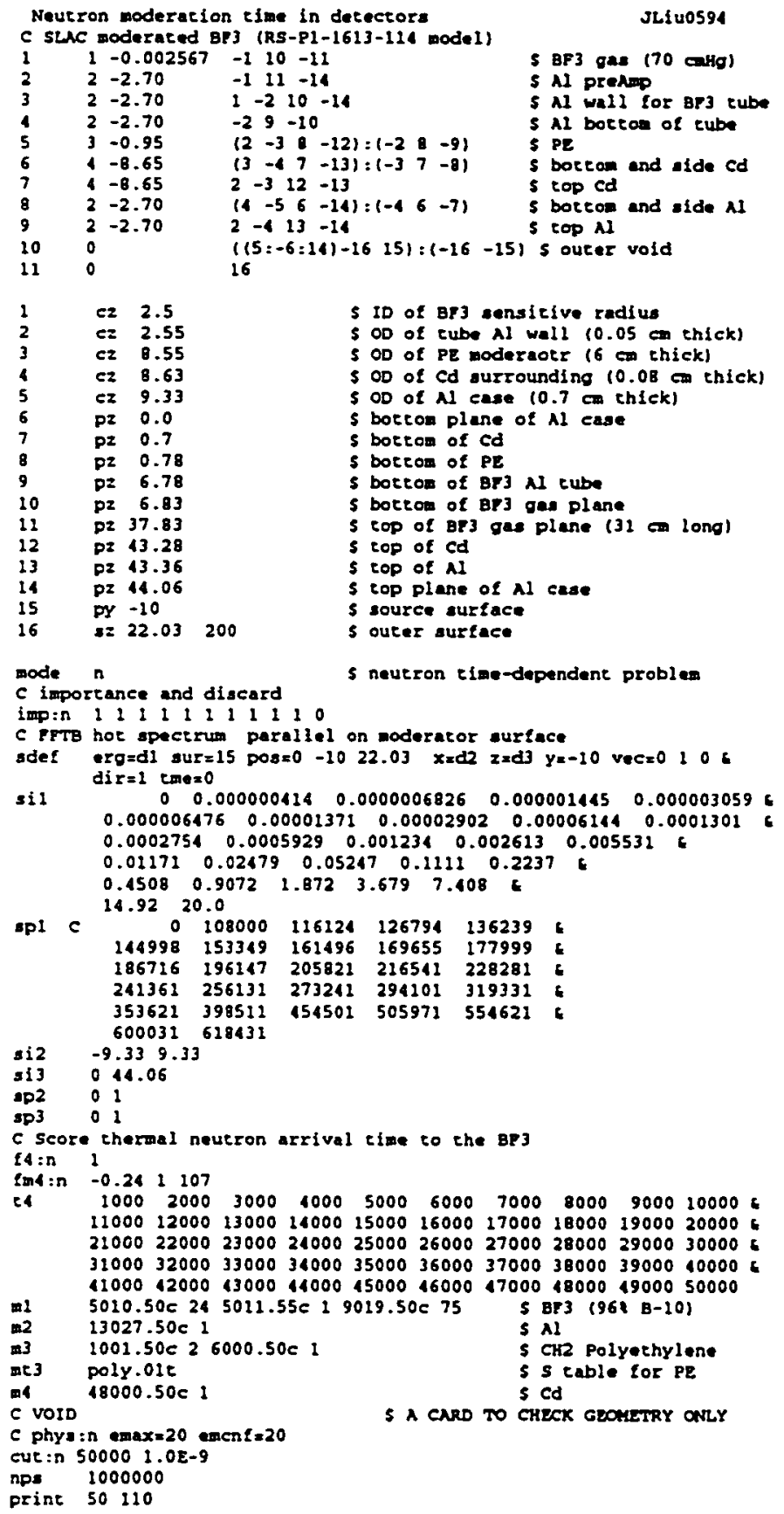




\section{Appendix B1}

MCNP input file of the Anderson-Braun remmeter for monoenergetic neutrons ( $1 \mathrm{MeV}$ ).

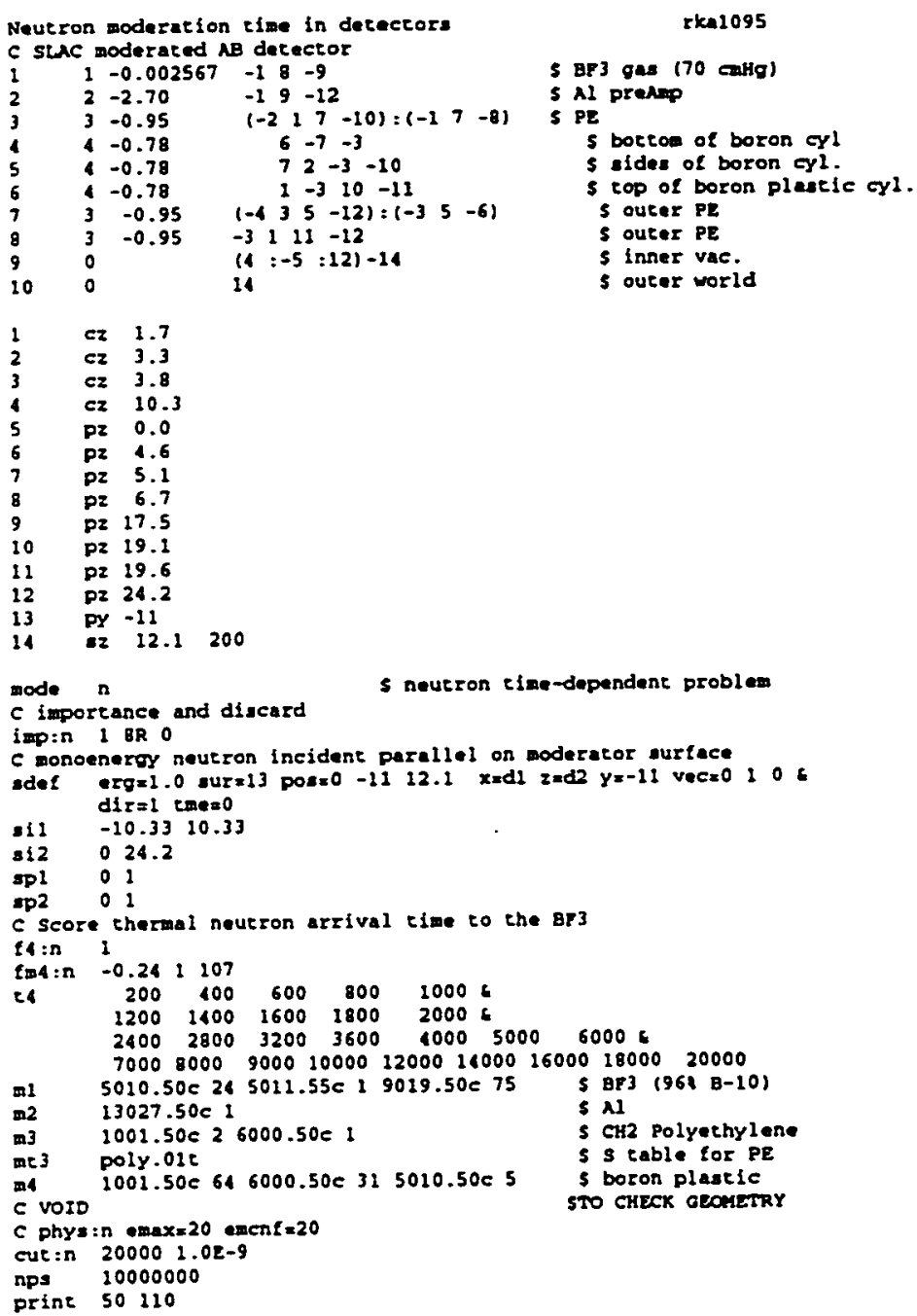




\section{Appendix B2}

MCNP input file of the Anderson-Braun remmeter for GRNs.

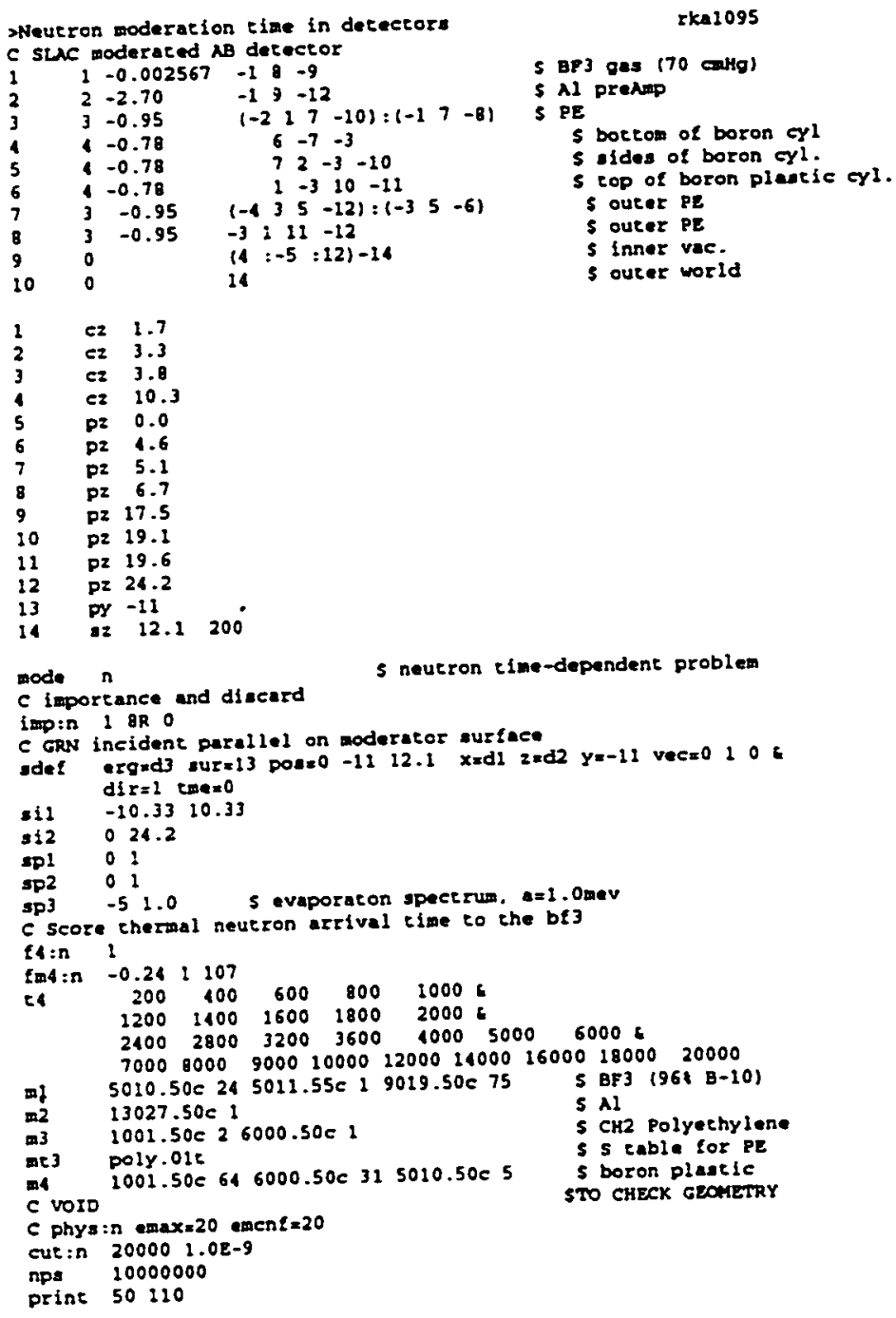




\section{Appendix B3}

MCNP input file of the Anderson-Braun remmeter for FFTB measured neutron spectrum

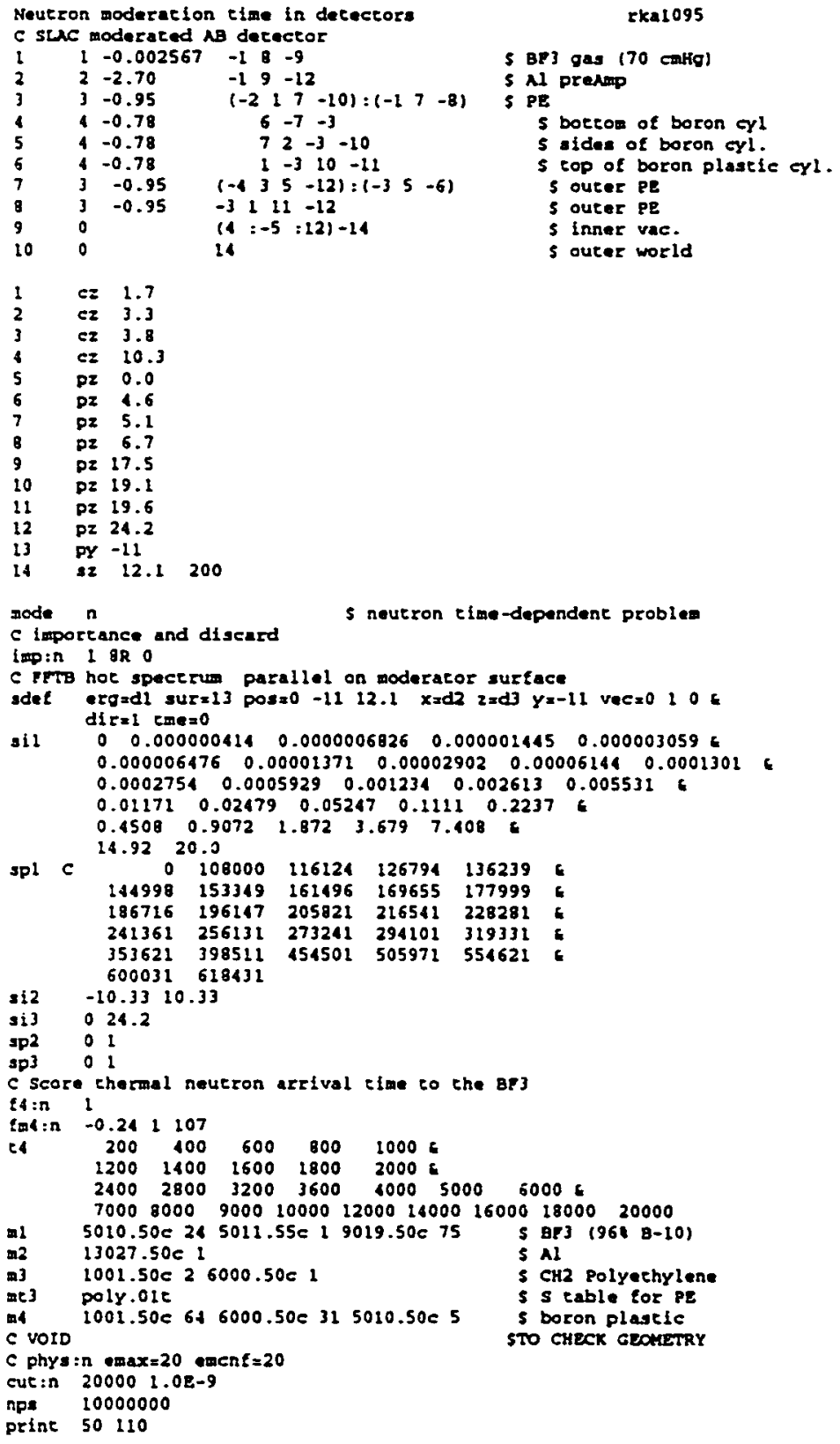




\section{Appendix Cl}

MCNP input file of the 2" Bonner Sphere for monoenergetic neutrons (1 MeV).

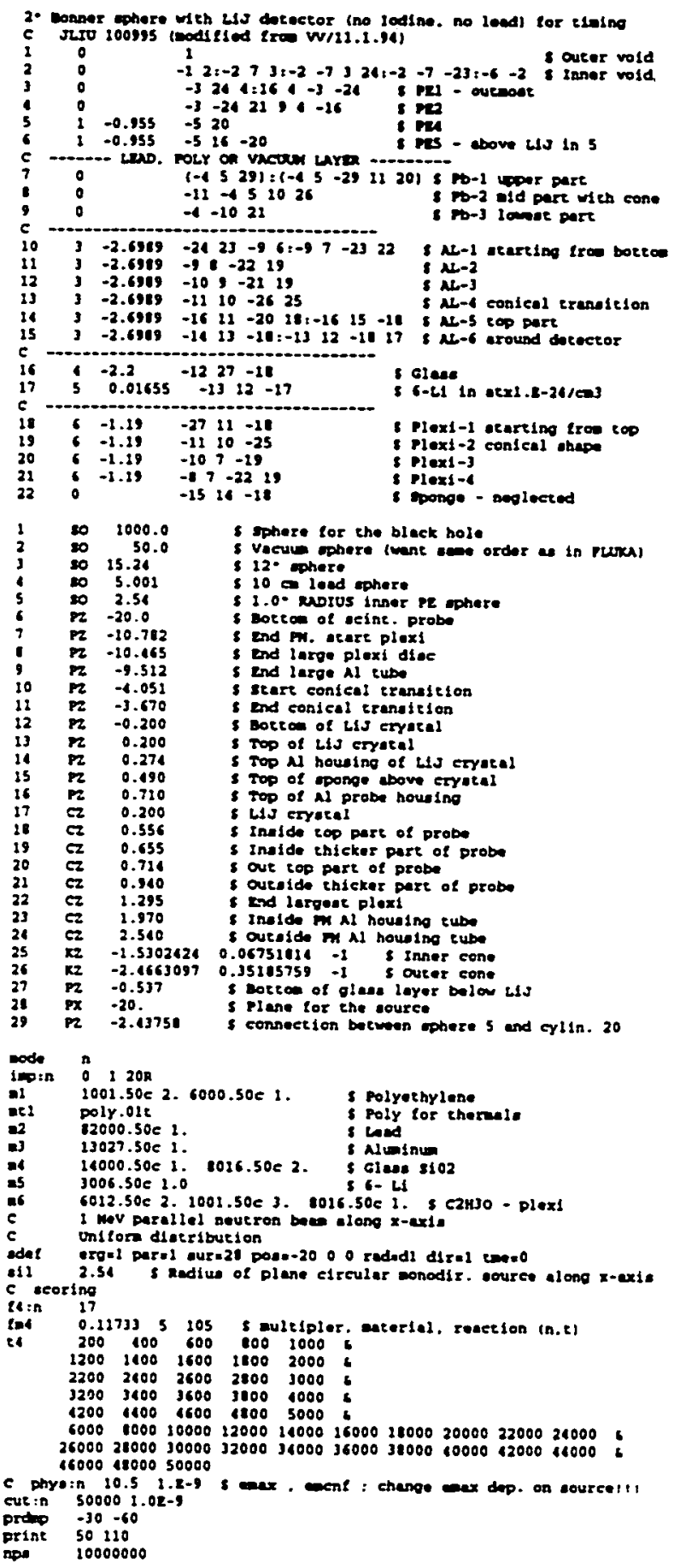




\section{Appendix C2}

MCNP input file of the 8" Bonner Sphere for GRNs.

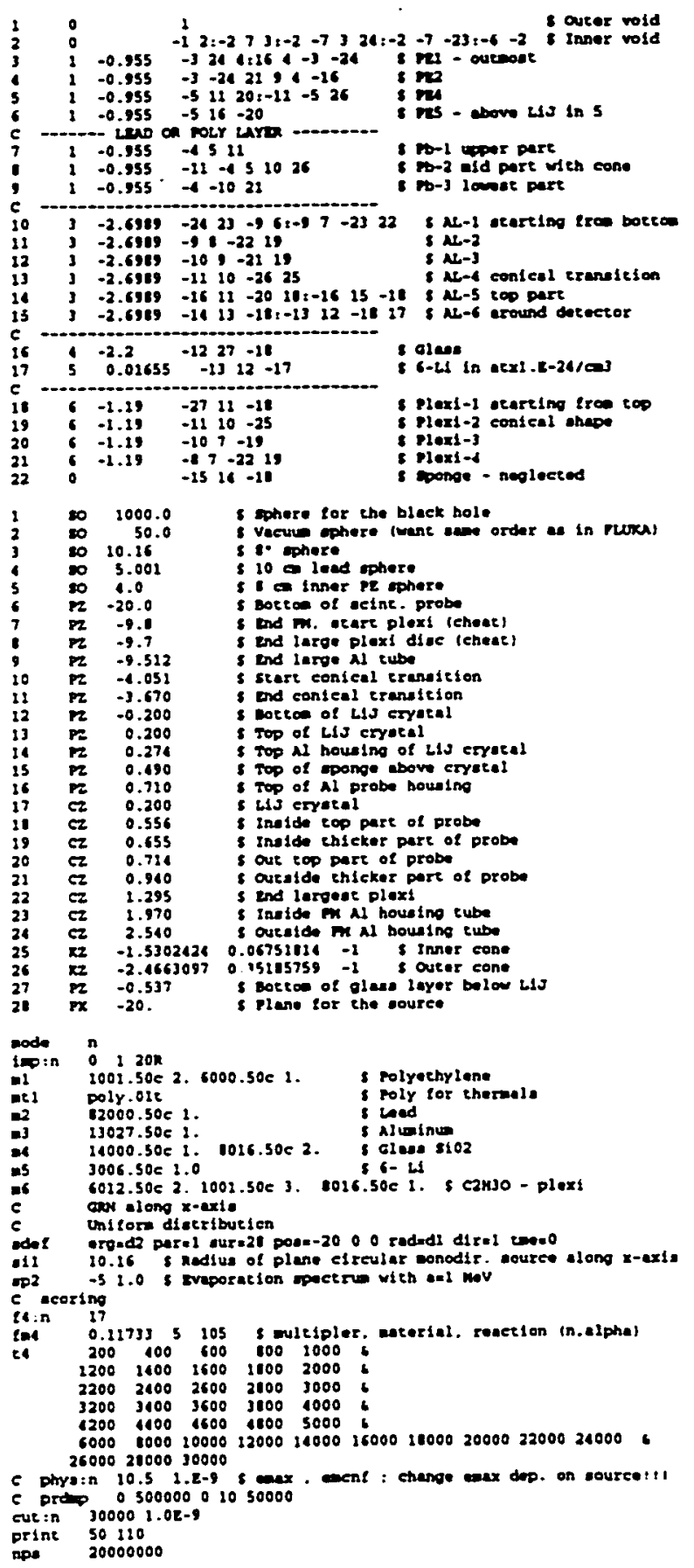




\section{Appendix C3}

MCNP input file of the 12" Bonner Sphere for FFTB measured neutron spectrum.

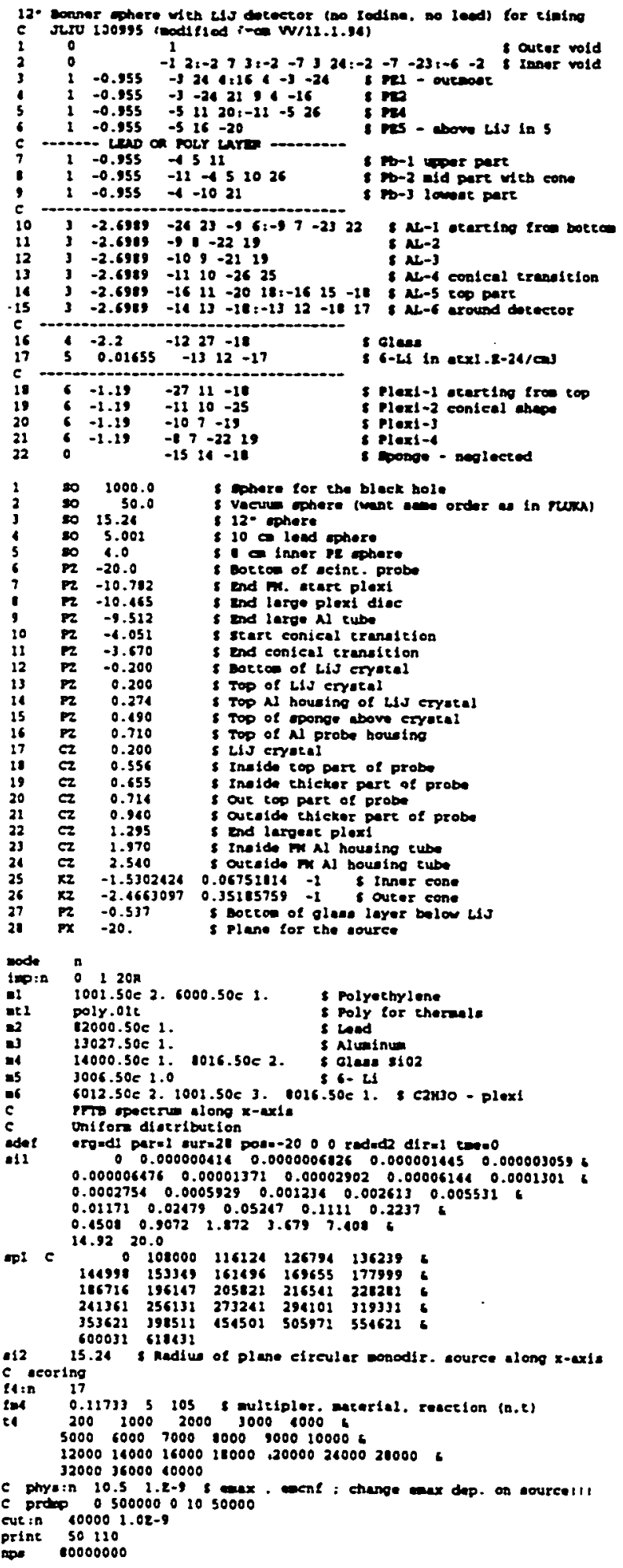




\section{Appendix D}

A portion of $\mathrm{MCNP}$ output file of moderated $\mathrm{BF}_{3}$ detector for $1 \mathrm{MeV}$ neutrons.

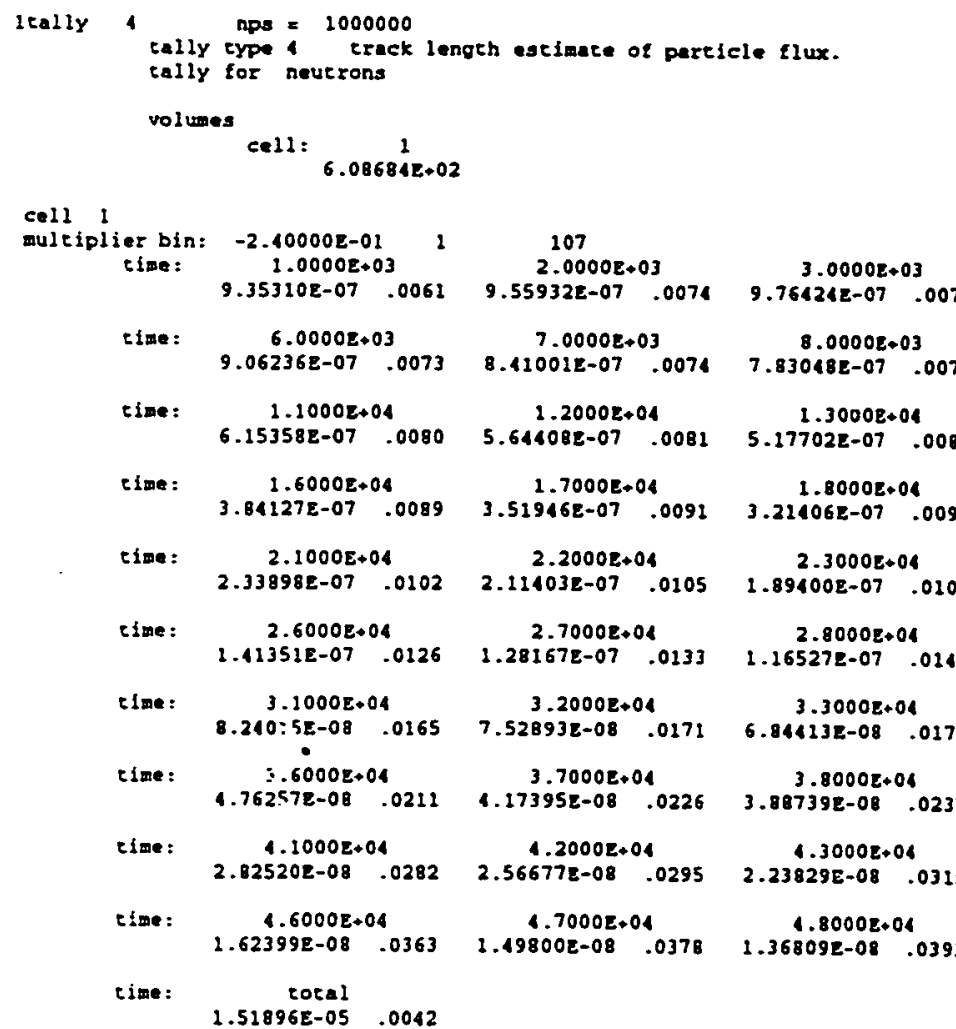

istatus of the atatistical checks used to form confidence intervals for che wean for each cally bin

cally result of statiatical checks for the tfe bin (the tirse check not pessed is listed) and error magnitude check for all bina

4 passed the 10 statistical checks for the cally fluctuation chare bin reault

passed all bin error check: 51 tally bins all have reletive errore lese than . 10 wth no zero bins

the 10 etatiatical check are only for the cally fluetuation chart bin and do not apply to orher eally bins. 


\section{Appendix E}

MCNP Calculations of response of $\mathrm{BF}_{3}$ detector and Anderson-Braun remmeter.

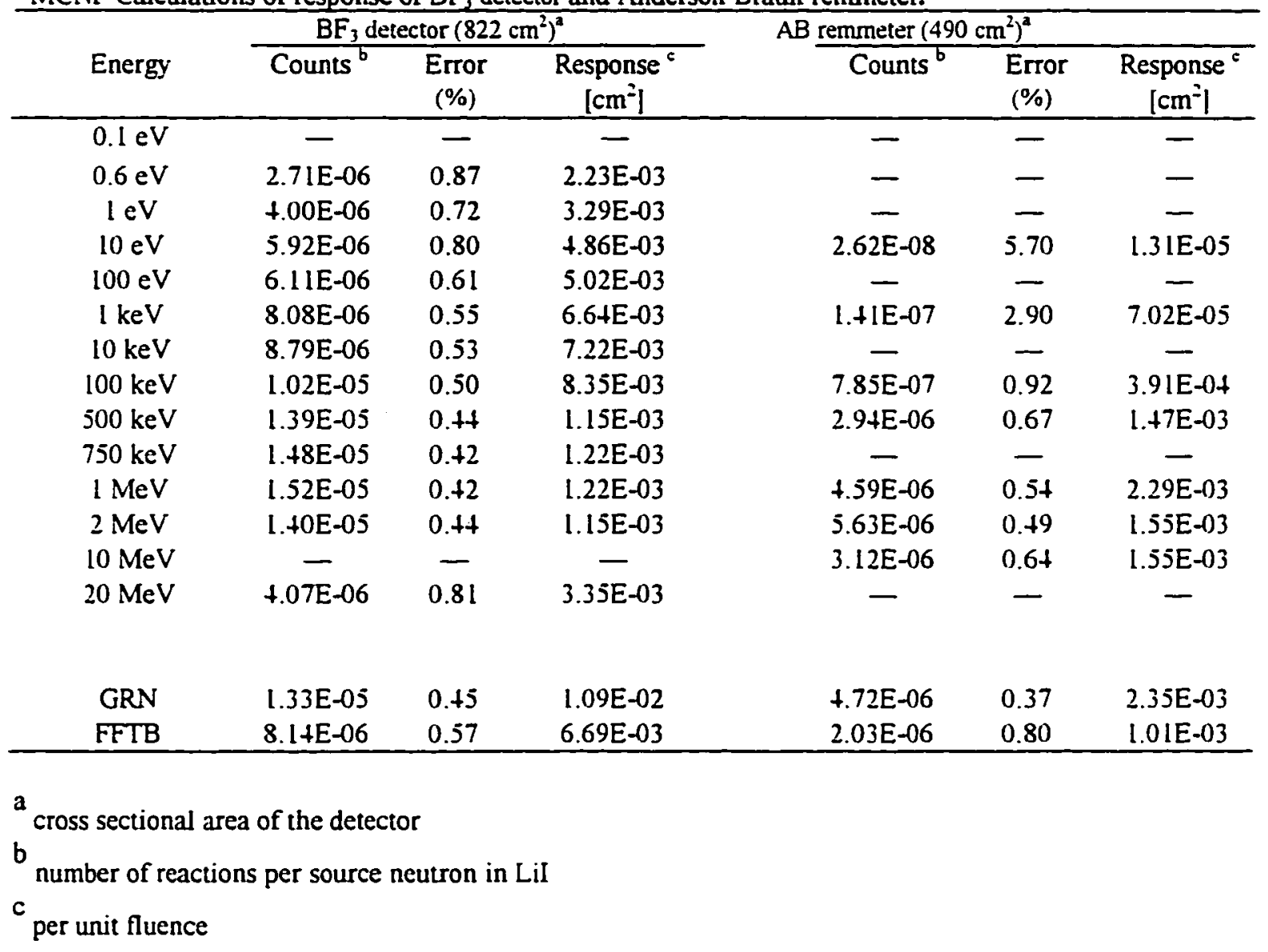

